\title{
PARTIAL ORDER EMBEDDINGS WITH CONVEX RANGE
}

\author{
JAMES HIRSCHORN
}

\begin{abstract}
A careful study is made of embeddings of posets which have a convex range. We observe that such embeddings share nice properties with the homomorphisms of more restrictive categories; for example, we show that every order embedding between two lattices with convex range is a continuous lattice homomorphism. A number of posets are considered; for example, we prove that every product order embedding $\sigma: \mathbb{N}^{\mathbb{N}} \rightarrow \mathbb{N}^{\mathbb{N}}$ with convex range is of the form
\end{abstract}

$$
\sigma(x)(n)=\left(\left(x \circ g_{\sigma}\right)+y_{\sigma}\right)(n) \text { if } n \in K_{\sigma},
$$

and $\sigma(x)(n)=y_{\sigma}(n)$ otherwise, for all $x \in \mathbb{N}^{\mathbb{N}}$, where $K_{\sigma} \subseteq \mathbb{N}, g_{\sigma}: K_{\sigma} \rightarrow \mathbb{N}$ is a bijection and $y_{\sigma} \in \mathbb{N}^{\mathbb{N}}$. The most complex poset examined here is the quotient of the lattice of Baire measurable functions, with codomain of the form $\mathbb{N}^{I}$ for some index set $I$, modulo equality on a comeager subset of the domain, with its 'natural' ordering.

\section{Contents}

1. Overview

1.1. Terminology

2. The quasi ordering of a monoid

2.1. Distributive laws

3. Order embeddings with preregular range

3.1. Preregularity

3.2. Order reflecting homomorphisms

3.3. Continuity

3.4. Bases of lattices

3.5. Extensions of embeddings

4. The partial orders

4.1. Power set algebras

4.2. Powers of $\mathbb{N}$

4.3. Category algebras

4.4. Baire functions into powers of $\mathbb{N}$ modulo almost always equality

4.5. Continuous functions into powers of $\mathbb{N}$

Date: January 2, 2007.

2000 Mathematics Subject Classification. Primary 06A06; Secondary 03E15, 03E40, 06A11, 06B30, 06F05, 54C35.

Key words and phrases. Partial order, embedding, convex, Scott topology, irrationals, Baire measurable, complete semilattice, monoid.

The author acknowledges the generous support of the Japanese Society for the Promotion of Science (JSPS Fellowship for Foreign Researchers, ID\# P04301). 


\section{Overview}

The abstract objects of study here are embeddings between arbitrary posets having preregular (e.g. convex, see definition 3.11) range. The main observation is that they are continuous with respect to a natural poset topology (namely the Scott topology). This observation is then applied to investigate embeddings with convex range between some specific classes of posets.

It turns out that the determination of these embeddings for various examples of posets generalizes known results about homomorphisms in more restrictive categories. For example, we show in 4.1 that every order embedding $\sigma: \mathcal{P}(X) \rightarrow \mathcal{P}(Y)$ with convex range is of the form $\sigma(a)=h[a] \cup b$ for some injection $h: X \rightarrow Y$ and some $b \subseteq Y$; this can be compared with the known fact that every continuous Boolean algebra monomorphism $\sigma: \mathcal{P}(X) \rightarrow \mathcal{P}(Y)$ is of the form $\sigma(a)=h^{-1}[a]$ for some partial surjection $h: Y \rightarrow X$.

By definition, an order embedding is an order preserving map (i.e. partial order homomorphism) that is an isomorphism onto its range. Thus for order theoretic structures, e.g. lattices and Boolean algebras, where the structure is completely determined by the ordering, an order embedding is in fact an isomorphism for the given structure onto its range. Hence characterizing these embeddings involves two aspects: determining the isomorphisms of the given structure; and determining the range of these embeddings. For example, it is a special case of the above mentioned known fact, that Boolean algebra isomorphisms between $\mathcal{P}(X)$ and $\mathcal{P}(Y)$ are of the form $\sigma(a)=h[a]$ for some bijection $h: X \rightarrow Y$. Thus the 'new result' in the above characterization concerns the range, namely that every order embedding $\sigma: \mathcal{P}(X) \rightarrow$ $\mathcal{P}(Y)$ with convex range has range equal to the interval $[\sigma(\emptyset), \sigma(X)]=\{a \subseteq Y$ : $\sigma(\emptyset) \subseteq a \subseteq \sigma(X)\}$. (Of course, the computation of the range in this example follows trivially from the definition of convexity, but in slightly more complex examples this computation can become difficult). However, this is an incomplete view of the situation where there is additional structure that is not purely order theoretic. Indeed many our examples are also monoids.

Write $\mathbb{N}$ for the set $\{0,1, \ldots\}$ of nonnegative integers. The usual (linear) partial ordering of $\mathbb{N}$ is $0<1<\cdots$. The example most important to us is the class of partial order embeddings between the irrationals $\mathbb{N}^{\mathbb{N}}$, i.e. the set of all functions from $\mathbb{N}$ into $\mathbb{N}$ (see e.g. [Hir06a]), with the product order. Indeed this paper is the second part of a series, where the third part [Hir06a] is entitled "Characterizing the quasi ordering of the irrationals by eventual dominance" (and it is the sequel to [Hir06b]), where order embeddings of the irrationals with convex range play a crucial role in characterizing the eventual dominance ordering (cf. \$4.6.4) in terms of the product order. 
More generally, we consider embeddings between the product orders $\mathbb{N}^{I}$ and $\mathbb{N}^{J}$ for arbitrary index sets $I$ and $J$. Thus for all $x, y \in \mathbb{N}^{I}$,

$$
x \leq y \quad \text { iff } \quad x(i) \leq y(i) \text { for all } i \in I .
$$

In this case we ascertained (cf. \$4.2) that every order embedding from $\mathbb{N}^{I}$ into $\mathbb{N}^{J}$ with convex range is in fact a monoid embedding for coordinatewise addition plus a constant in $\mathbb{N}^{J}$.

Further generalization was desired to function spaces with the irrationals as the codomain. Let $X$ and $Y$ be topological spaces. We let Baire $(X, Y)$ denote the family of all Baire measurable functions from $X$ into $Y$. In 4.4 we consider quotients of Baire $\left(X, \mathbb{N}^{\mathbb{N}}\right)$ (where $\mathbb{N}^{\mathbb{N}}$ has the product topology) over the equivalence relation of "almost always" equality $=_{a \mathrm{a}}$ :

$$
f={ }_{\text {aa }} g \quad \text { if } \quad f(z)=g(z) \quad \text { for almost all } z \in X,
$$

or in other words for comeagerly many $z \in X$; where $\operatorname{Baire}\left(X, \mathbb{N}^{\mathbb{N}}\right) /={ }_{\text {aa }}$ is given the order $\leq_{\text {aa }}$ induced by $\leq$ :

$$
[f] \leq_{\mathrm{aa}}[g] \quad \text { iff } \quad f(z) \leq g(z) \quad \text { for almost all } z \in X .
$$

The embeddings of Baire $\left(X, \mathbb{N}^{\mathbb{N}}\right) /={ }_{\text {aa }}$ into Baire $\left(Y, \mathbb{N}^{\mathbb{N}}\right) /={ }_{\text {aa }}$ with convex range are described precisely, where, as far as we know, even the isomorphism structure was not previously known. This involves characterizing embeddings with convex range of category algebras of topological spaces, or equivalently the regular open algebras of these spaces, in $\$ 4.3$. We note that there are some subtleties in generalizing to arbitrary index sets, i.e. to $\operatorname{Baire}\left(X, \mathbb{N}^{I}\right) /=_{\text {aa }}$, that are addressed there.

Our interest in quotients of Baire functions stems from set theoretic forcing. For example, for an index set $I$ in the 'reference model' - called the ground model in the terminology of set theoretic forcing, it is well known that every member of $\mathbb{N}^{I}$ in the extension of this model obtained by Cohen forcing, is determined by a member of $\operatorname{Baire}\left(\mathbb{R}, \mathbb{N}^{I}\right) /={ }_{\text {aa }}$ in the ground model. Whilst in the other direction, we shall use set theoretic forcing to prove that (Baire $\left(X, \mathbb{N}^{I}\right) /=_{\mathrm{aa}}, \leq_{\mathrm{aa}}$ ) is a complete semilattice satisfying many of the same properties as $\mathbb{N}^{I}$ (theorem 4.40). This can be generalized extensively; for example, for $S$ in the ground model, every member of $\mathcal{P}(S)$ in the Cohen model corresponds to a member of Baire $(\mathbb{R}, \mathcal{P}(S)) /={ }_{\text {aa }}$ (cf. $\sqrt[4.6 .1]{ }$ ), and by replacing "almost always" with "almost everywhere" in the measure theoretic sense, we obtain a correspondence with random forcing (cf. \$4.6.2).

In $\$ 2$ we study a standard association of a quasi order to every monoid. We are especially interested in those monoids - we call them lattice monoids - where the associated quasi order is in fact a lattice. Then various infinite distributive laws are examined for these lattice monoids. The main purpose of this section for the present paper, is that it allows us in $\$ 3.5$ to use algebraic methods to extend embeddings from a suitably dense subset of some lattice monoid to the entire lattice (cf. corollary 3.98). This in turn is applied in $\$ 4.5$ to use our description in $\$ 4.4$ of the embeddings of $\operatorname{Baire}\left(X, \mathbb{N}^{I}\right) /={ }_{\text {aa }}$ into $\operatorname{Baire}\left(Y, \mathbb{N}^{J}\right) /={ }_{\text {aa }}$ with convex range in order to obtain a precise description of the class of embeddings with convex range of $C\left(X, \mathbb{N}^{I}\right)$ into $C\left(Y, \mathbb{N}^{J}\right)$. That is, the family of continuous functions ordered 
pointwise. We also see examples (e.g. lemma 3.16, theorem 3.59) of how algebraic properties of subsets of lattices may entail order theoretic regularity properties.

The general theory is in $\$ 3$, including the Scott continuity of embeddings with preregular range, and numerous consequences of this result. In $§ 3.4$, we study various notions of denseness in a lattice, and this is applied in $\$ 3.5$ to extend homomorphisms from suitably dense subsets of a lattice while preserving various desirable properties.

Further directions for the Boolean algebra $\left(\mathcal{P}(\mathbb{N}) /\right.$ Fin, $\left.\subseteq^{*}\right)$ and its close relative the lattice $\left(\mathbb{N}^{\mathbb{N}} /\right.$ Fin, $\left.\leq^{*}\right)$ are suggested in $\$ 4.6 .3$ and $\$ 4.6 .4$.

1.1. Terminology. A homomorphism refers to an arrow (i.e. morphism) of the intended category, while an isomorphism is an invertible homomorphism. For a concrete category $(C, U)$, we say that an embedding is a homomorphism $h: c \rightarrow d$ for which there exists some invertible homomorphism $g$ with domain $c$ such that $U h(x)=U g(x)$ for all $x \in U c$. Thus in a category where the homomorphisms are functions, an embedding is a homomorphism $f: X \rightarrow Y$ that is an isomorphism onto its range, i.e. $\operatorname{ran}(f)$ is an object of the category and $f^{\prime}: X \rightarrow \operatorname{ran}(f)$ given by $f^{\prime}(x)=f(x)$ is an isomorphism. Monomorphisms (i.e. monics) refer to homomorphisms that are left cancellative under composition, i.e. $f$ is a monomorphism iff $f \circ g=f \circ h$ implies $g=h$ for all $g, h$ with codomain equal to the domain of $f$. And epimorphisms (i.e. epis) refer to homomorphisms that are right cancellative. In all of the concrete categories considered below, monomorphisms are simply the injective homomorphisms, and in all of these categories with the exception of the monoids, epimorphisms are precisely the surjective homomorphisms.

A quasi order (also often called a preorder) is a pair $(O, \leq)$ where $\leq$ is a reflexive and transitive relation on $O$. For a quasi order $(O, \leq)$, we write $<$ for the relation defined by $p<q$ if $p \leq q$ and $q \not \leq p$. Note this disagrees with another usage where $p<q$ iff $p \leq q$ and $p \neq q$. A poset (partial order) is a quasi order where the relation is also antisymmetric (i.e. $p \leq q$ and $q \leq p$ imply $p=q$ ). In a poset $(P, \leq), p<q$ iff $p \leq q$ and $p \neq q$. A poset with a minimum element is called a pointed poset. Note that if $(O, \leq)$ is a quasi order (poset) then for every subset $A \subseteq O,(A, \leq)$ is also a quasi order (poset). We call it a quasi suborder (subposet). In the category of quasi orders, the homomorphisms are order preserving maps, i.e. for two quasi orders $(O, \leq)$ and $(Q, \lesssim), \sigma: O \rightarrow Q$ is order preserving if $p \leq q$ implies $\sigma(p) \lesssim \sigma(q)$ for all $p, q \in O$. Thus isomorphisms are bijections that are both order preserving and order reflecting, where $\sigma: O \rightarrow Q$ is order reflecting if $\sigma(p) \lesssim \sigma(q)$ implies $p \leq q$ for all $p, q \in O$. A subset $A \subseteq O$ is called bounded above, or just bounded, if it has an upper bound, i.e. some $p \in O$ such that $a \leq p$ for all $a \in A$. And we say that $A$ is bounded below if it has a lower bound, i.e. some $q \in O$ such that $q \leq a$ for all $a \in A$.

The class of posets is viewed as a full subcategory of the quasi orders. Notice that for $(P, \leq)$ a poset and $(Q, \lesssim)$ a quasi order, $\sigma: P \rightarrow Q$ is an embedding iff it is both order preserving and reflecting. We write $\bigvee A$ for the supremum, i.e. minimum upper bound, of a subset $A \subseteq P$ (which may or may not exist), and we write $\bigwedge A$ for the infimum, i.e. maximum lower bound. We write $a \vee b$ and $a \wedge b$ for $\bigvee\{a, b\}$ 
and $\bigwedge\{a, b\}$, respectively. A function $\sigma: P \rightarrow Q$ between two posets is called joinpreserving if $\sigma(p \vee q)=\sigma(p) \vee \sigma(q)$ whenever $p \vee q$ exists, for all $p, q \in P$ (i.e. if $p \vee q$ exists then so does $\sigma(p) \vee \sigma(q)$ satisfying the equation). The dual notion is meet-preserving.

We take a join semilattice, which we also just call a semilattice, to be a poset $(L, \leq)$ such that $\vee$ is a binary operation on $L$, i.e. $p \vee q$ exists for all $p, q \in L$. And a meet semilattice is defined dually. A lattice is a poset that is both a semilattice and a meet semilattice. A subsemilattice of a semilattice $(L, \leq)$ is a semilattice $(A, \leq)$, where $A \subseteq L$, such that $(A, \leq) \models\ulcorner c=a \vee b\urcorner$ iff $(L, \leq) \models\ulcorner c=a \vee b\urcorner$, i.e. the supremum computed in the order $(A, \leq)$ agrees with the supremum taken in $L$. Meet subsemilattices and sublattices are defined analogously. Note that a subset $A \subseteq L$ may be a lattice as a subposet of $(L, \leq)$, without being a sublattice. The homomorphisms of the category of semilattices are the join-preserving functions, while the homomorphisms of the category of meet semilattices are the meet-preserving functions. Note that these are both subcategories of the poset category, because $p \vee q$ and $p \wedge q$ do not exist when $p \leq q, q \leq p$ and $q \neq p$, and because join/meet semilattice homomorphisms are order preserving, as $p \leq q$ iff $p \vee q=q$ iff $p \wedge q=p$. The category of lattices is the intersection of the categories of join and meet semilattices, i.e. the homomorphisms are the functions that are both join and meet-preserving. It is easy to find a counterexample showing that a quasi order homomorphism between two lattices need not be a lattice homomorphism (i.e. lattices are not a full subcategory of the quasi orders). On the other hand, since the lattice operations are obviously determined by the ordering of the lattice, a quasi order isomorphism between two lattices (equivalently, a poset isomorphism) is in fact a lattice isomorphism. Take note that lattice embeddings are the same thing as lattice monomorphisms (and similarly for join/meet semilattice embeddings), i.e. they are the injective lattice homomorphisms (this is not true of the category of posets). Moreover:

Proposition 1.1. A lattice homomorphism $\sigma$ is an embedding iff it is strictly order preserving, i.e. $p<q$ implies $\sigma(p)<\sigma(q)$.

By a complete semilattice we mean a join semilattice $(L, \leq)$ such that $\bigvee A$ exists whenever $A \subseteq L$ is bounded. Note that a complete semilattice is pointed, with minimum element $\bigvee \emptyset$, which we denote by 0 . Notice also that a complete semilattice is in fact a lattice, and moreover $\bigwedge A$ exists whenever $A \neq \emptyset$ (see e.g. [DP02]). A complete lattice is a lattice such that $\bigvee A$ and $\bigwedge A$ exist for every subset $A \subseteq L$. By adding a top element to any complete semilattice one obtains a complete lattice. A lattice is called bounded if it has both a maximum and minimum element; we denote the maximum element by 1 .

We take a Boolean algebra to be a bounded lattice $(B, \leq)$ such that every $p \in B$ has a complement, which we write as $-p$, satisfying $p \wedge-p=0$ and $p \vee-p=1$. Homomorphisms in the category of Boolean algebras are lattice homomorphisms that preserve complements (and thus also preserve 0 and 1). Since the Boolean algebra operations are completely determined by the order, every poset isomorphism between two Boolean algebras is in fact a Boolean algebra isomorphism. In the category of Boolean algebras embeddings and monomorphisms coincide. 
Recall that a monoid $(M, \cdot)$ is a semigroup that has an identity. We do not need to specify the identity because it is uniquely determined; we denote it by $e$. A monoid is cancellative if $a \cdot b=a \cdot c$ implies $b=c$ and $b \cdot a=c \cdot a$ implies $b=c$, for all $a, b, c \in M$. The inverse of $a \in M$, when it exists, is denoted $a^{-1}$; more generally, we write $a \cdot b^{-1}$ for the element $c \in M$ such that $a=c \cdot b$, if it exists. It is uniquely determined so long as $M$ is cancellative. We write $(M,+)$ when dealing with a commutative monoid; use - to denote the inverse; and we use 0 to denote the identity. A monoid homomorphism is map preserving both the monoid operation and the identity. In the category of monoids embeddings and monomorphisms both coincide with injective homomorphisms. When we say that a subset $S$ of a commutative monoid is closed under subtraction we of course mean that $a-b \in S$ whenever $a, b \in S$ and $a-b$ exists. Since every cancellative commutative monoid embeds into an Abelian group (lemma 2.13), a submonoid of a cancellative commutative monoid that is closed under subtraction can be viewed as a 'subgroup'.

Proposition 1.2. Let $(G,+)$ be an Abelian group, and $M \subseteq G$ be a submonoid. Then a subsemigroup $S \subseteq M$ is closed under subtraction iff $\langle S\rangle \cap M=S$, where $\langle S\rangle$ denotes the subgroup generated by $S$.

Proof. Since $S$ is a subsemigroup of an Abelian group, $\langle S\rangle=S-S$. It immediately follows that $\langle S\rangle \cap M=S$ iff $S$ is closed under subtraction.

Recall that a binary relation $R$ on a set $X$ is a congruence on some $n$-ary relation $S$ on $X$, if $S\left(x_{0}, \ldots, x_{n-1}\right)$ iff $S\left(x_{0}^{\prime}, \ldots, x_{n-1}^{\prime}\right)$, whenever $x_{k} R x_{k}^{\prime}$ for all $k=0, \ldots, n-1$; and it is a congruence on some $n$-ary function $f$ on $X$, if $f\left(x_{0}, \ldots, x_{k-1}\right) R f\left(x_{0}^{\prime}, \ldots, x_{k-1}^{\prime}\right)$ whenever $x_{k} R x_{k}^{\prime}$ for all $k=0, \ldots, n-1$. A congruence $\sim$ on some quasi order $(O, \leq)$, i.e. a congruence on $\leq$, that is moreover an equivalence relation on $O$ is an orderable partition of $(O, \leq)$ in the terminology of DR81, and it is called so because $\leq$ determines a well defined ordering of the quotient $O / \sim$ via $[p] \leq[q]$ if $p \leq q$. A lattice congruence on some lattice $(L, \leq)$ means a relation on $L$ that is a congruence for both of the binary lattice operations. A lattice congruence $\sim$ that is also an equivalence relation induces a lattice structure on $L / \sim$, with $[a] \wedge[b]=[a \wedge b]$ and $[a] \vee[b]=[a \vee b]$. And a Boolean algebra congruence is a lattice congruence that is also a congruence for the unary complement operator. Of course a Boolean algebra congruence that is an equivalence relation on $(B, \leq)$ determines a quotient Boolean algebra $B / \sim$ with $-[a]=[-a]$.

Similarly, a monoid congruence for a monoid $(M, \cdot)$ is a congruence on the monoid operation $\cdot$, and an equivalence relation $\sim$ that is a congruence on $\cdot$ determines a quotient monoid $(M / \sim, \cdot)$.

\section{THE QUASI ORDERING OF A MONOID}

All monoids have a naturally associated quasi ordering. This is well known, e.g. [Kan69] to give one example. In this section we examine some basic properties of this associated quasi ordering, and introduce the notion of a lattice monoid. Then we focus on distributivity, where we prove that a large class of monoids satisfy certain infinitary distributive laws; for example, in corollary 2.30 we prove that in 
every cancellative commutative monoid, addition distributes over arbitrary suprema. As far as the examples of $\$ 4$ are concerned, the results of this section are only applied to the lattice monoid of $\$ 4.5$. However, lattice monoids will play a bigger role in the sequel to this paper.

Definition 2.1. A monoid $(M, \cdot)$ has an associated quasi order $\leq_{(M, \cdot)}$ on $M$ defined by

$$
x \leq_{(M, \cdot)} y \quad \text { if } \quad x \cdot a=y \text { for some } a \in M .
$$

Proposition 2.2. If $(M, \cdot)$ is a monoid the the relation defined in equation (2.1) is a quasi ordering.

Proof. It is reflexive because $(M, \cdot)$ has an identity, and it is transitive because $(M, \cdot)$ is a semigroup.

Proposition 2.3. For any monoid, the identity is a minimum element of the associated quasi order.

Proof. For all $a \in M, e \cdot a=a$ implies $e \leq_{(M, \cdot)} a$.

The quasi order associated with a cancellative monoid has no maximal elements unless the monoid is a group, in which case the associated quasi order is the complete quasi order.

Lemma 2.4. Let $(M, \cdot)$ be a cancellative monoid. If $\left(M, \leq_{(M, \cdot)}\right)$ has a maximal element then $(M, \cdot)$ is a group.

Proof. Let $a \in M$ be a maximal element. Take any $b \in M$. Then $a \leq_{(M, \cdot)} a \cdot b$ implies $a \cdot b \leq_{(M, \cdot)} a$ and thus $a \cdot b \cdot c=a$ for some $c \in M$. Hence $b \cdot c=e$ by cancellativity. The existence of right inverses for all elements entails that $(M, \cdot)$ is a group.

Proposition 2.5. Let $(M, \cdot)$ be a monoid. Then $b \leq_{(M, \cdot)} c$ implies $a \cdot b \leq_{(M, \cdot)} a \cdot c$, for all $a, b, c \in M$.

Proof. Assuming $b \leq_{(M, \cdot)} c$, there exists $d$ such that $b \cdot d=c$. Thus $(a \cdot b) \cdot d=$ $a \cdot(b \cdot d)=a \cdot c$.

We are mostly interested in commutative monoids so that we at least have monotonicity. Indeed, the category of positively quasi ordered commutative monoids is widely studied in Ordered Algebra, see e.g. WWeh92] where they are named POM's, and the quasi order $\leq_{(M,+)}$ associated with a commutative monoid is the minimal quasi ordering such that $\left(M,+, \leq_{(M,+)}\right)$ is a POM.

Proposition 2.6. Let $(M,+)$ be a commutative monoid. Then $a \leq_{(M,+)} b$ implies $a+c \leq_{(M,+)} b+c$, for all $a, b, c \in M$.

Proof. Write $a+d=b$. Then $(a+c)+d=a+d+c=b+c$.

Definition 2.7. A poset monoid is a monoid $(M, \cdot)$ whose associated quasi order is in fact a partial order. 
Proposition 2.8. Every poset monoid is pointed with e the minimum element.

\section{Proof. Proposition 2.3,}

Lemma 2.9. Poset monoids do not have invertible elements besides the identity.

Proof. Suppose that $a \in M$ is invertible. Then $a \cdot a^{-1}=e$ implies $a \leq_{(M, \cdot)} e$, and $e \leq_{(M, \cdot)} a$ by proposition 2.3 . Thus $a=e$ by antisymmetry.

Corollary 2.10. Cancellative poset monoids do not have maximal elements, with the exception of the singleton monoid $\{e\}$.

Proof. Lemmas 2.4 and 2.9.

Definition 2.11. A monoid $(M, \cdot)$ is called a semilattice monoid if the associated quasi order is actually a semilattice, and it is called a lattice monoid if the associated quasi order is moreover a lattice.

Remark 2.12. Note that in a semilattice monoid $0=e$ by proposition 2.8. Thus our usage of 0 as the additive identity in a commutative monoid is consistent with its usage as the minimum element of a pointed semilattice.

We recall the following basic fact and provide a proof.

Lemma 2.13. A commutative monoid is embeddable (as a monoid) in some Abelian group iff it is cancellative.

Proof. Let $(M,+)$ be commutative and cancellative. Let $M^{-}$denote the subgroup of invertible elements of $M$. Note that commutativity entails that $N=\left(M \backslash M^{-}\right) \cup\{0\}$ is a submonoid of $(M,+)$. Now consider the product monoid $M \times N$ modulo the relation defined by

$$
(a, b) \sim(\bar{a}, \bar{b}) \quad \text { if } \quad \exists r, \bar{r} \in N \quad a+r=\bar{a}+\bar{r} \text { and } b+r=\bar{b}+\bar{r} .
$$

Note that $\sim$ is an equivalence relation: $0 \in N$ implies reflexivity, symmetry is obvious, and we have transitivity because supposing $(a, b) \sim(\bar{a}, \bar{b})$ and $(\bar{a}, \bar{b}) \sim(\overline{\bar{a}}, \overline{\bar{b}})$, $a+r=\bar{a}+\bar{r}$ and $\bar{a}+s=\overline{\bar{a}}+\bar{s}$ which with commutativity imply $a+r+s=\bar{a}+\bar{r}+s=$ $\overline{\bar{a}}+\bar{r}+\bar{s}$, and similarly $b+r+s=\overline{\bar{b}}+\bar{r}+\bar{s}$. And using commutativity similarly, $\sim$ is a congruence for addition (cf. \$1.1). Therefore $(M \times N) / \sim$ is a quotient monoid. Observe that

$$
[b, b]=[0,0] \text { for all } b \in N,
$$

and of course $[0,0]$, i.e. $[(0,0)]$, is the identity.

Claim 2.14. $((M \times N) / \sim,+)$ is an Abelian group.

Proof. It is commutative because it is the quotient of a commutative monoid. Take $[a, b]$ in the quotient. We prove that it has an inverse $-[a, b]$. First suppose that $a \in M^{-}$. Then $-[a, b]=[-a+b, 0]$ because $[a, b]+[-a+b, 0]=[b, b]=[0,0]$ by (2.3). Otherwise $-[a, b]=[b, a]$ because $[a, b]+[b, a]=[a+b, a+b]$.

Claim 2.15. $a \mapsto[a, 0]$ is a monomorphism between $M$ and $(M \times N) / \sim$. 
Proof. $a+b$ is mapped to $[a+b, 0]=[a, 0]+[b, 0]$ and hence it a monoid homomorphism. Now suppose $[a, 0]=[b, 0]$. Then there are $r$ and $s$ in $N$ such that $a+r=b+s$, and $0+r=0+s$, i.e. $r=s$. And by the cancellative property $a+r=b+r$ implies $a=b$ as needed.

Claims 2.14 and 2.15 establish one direction of the lemma, and it is clear that a noncancellative monoid cannot be embedded in a group.

This is used to show that cancellative commutative monoids satisfy monotonicity for subtraction.

Proposition 2.16. Let $(M,+)$ be a cancellative commutative monoid. Whenever $a-c$ exists, $b \leq_{(M,+)} c$ implies $a-b$ exists and $a-b \geq_{(M,+)} a-c$, for all $a, b, c \in M$.

Proof. By lemma 2.13, we may assume that $M$ is a submonoid of some Abelian group $(G,+)$. Now $b \leq_{(M,+)} c$ implies $b+d=c$ for some $d \in M$, and thus $(a-c)+d=(a-(b+d))+d=a-b$ as wanted, by the basic properties of a group.

Proposition 2.17. Let $(M,+)$ be a cancellative commutative monoid. Whenever $a-c$ exists, $a \leq_{(M,+)} b$ implies $b-c$ exists and $a-c \leq_{(M,+)} b-c$, for all $a, b, c \in M$.

Proof. Write $a+d=b$. Then since by lemma 2.13 we can assume we are working inside an Abelian group, $(a-c)+d=(a+d)-c=b-c$ by basic properties of an Abelian group.

2.1. Distributive laws. Recall that a lattice $(L, \vee, \wedge)$ is distributive if $a \wedge(b \vee c)=$ $(a \wedge b) \vee(a \wedge c)$ for all $a, b, c \in L$, and that:

Proposition 2.18. $(L, \vee, \wedge)$ is distributive iff $a \vee(b \wedge c)=(a \vee b) \wedge(a \vee c)$ for all $a, b, c \in L$.

Definition 2.19. We say that a monoid $(M, \cdot)$ is left --distributive over $\vee$, or simply left $(\cdot, \vee)$-distributive, if

$$
a \cdot(b \vee c)=(a \cdot b) \vee(a \cdot c) \quad \text { whenever } b \vee c \text { exists }
$$

for all $a, b, c \in M$, and it is right--distributive over $\vee$ if

$$
(a \vee b) \cdot c=(a \cdot c) \vee(b \cdot c) \quad \text { whenever } a \vee b \text { exists }
$$

for all $a, b, c \in M$, while it is --distributive over $\vee$ (or just $(\cdot, \vee)$-distributive) if it is both left and right -distributive over $\vee$. The definition of --distributivity over $\wedge$ is exactly analogous.

Let us say that it is $(\cdot, \mathrm{M})$-distributive if it is both $(\cdot, \vee)$-distributive and $(\cdot, \wedge)$ distributive. We will say that a lattice monoid is distributive if it is both $(\cdot, \bigvee)$ distributive and distributive as a lattice.

Note that while we are mostly interested in --distributivity over $\vee$ in semilattice monoids, and -distributivity over $\wedge$ is meet semilattice monoids, the definition does make sense for arbitrary monoids.

Of course, the notions of left $(+, \vee)$-distributivity, right $(+, \vee)$-distributivity and $(+, \vee)$-distributivity all coincide for any commutative monoid $(M,+)$, and similarly for + -distributivity over $\wedge$. 
Lemma 2.20. Let $(M,+)$ be a commutative $(+, \wedge)$-distributive monoid. Then $a \wedge b=0$ implies $a \vee b=a+b$, for all $a, b \in M$.

Proof. By commutativity, $a, b \leq a+b=b+a$. Suppose $a \wedge b=0$ and $d \geq a, b$. Let $c$ satisfy $a+c=d$. Then by right $(+, \wedge)$-distributivity, $c=(a \wedge b)+c=$ $(a+c) \wedge(b+c)=d \wedge(b+c) \geq b$ implies $d \geq a+b$ by proposition 2.5.

Lemma 2.21. Let $(M,+)$ be a commutative cancellative + -distributive monoid over $\vee$ and $\wedge$. Then $a \wedge c=0$ and $b \wedge c=0$ imply $(a+b) \wedge c=0$.

Proof. Suppose that $a \wedge c=b \wedge c=0$, and take $d \leq(a+b), c$. By lemma 2.20 and +-distributivity over $\vee, a+b+d=a+(b \vee d)=(a+b) \vee(a+d)$, and similarly $a+b+d=(a+b) \vee(b+d)$. Thus

$$
a+b+d=(a+b) \vee(a+d) \vee(b+d) .
$$

But we also have $a+b+2 d=(a+d)+(b+d)=(a \vee d)+(b \vee d)=(a+b) \vee$ $(a+d) \vee(d+b) \vee(d+d)$ by distributivity over $\vee$. Therefore, as $2 d \leq a+b+d$ since $d \leq a+b$, we obtain $a+b+2 d=a+b+d$, and thus $d=0$ by cancellativity.

\subsubsection{Infinite distributive laws.}

Notation 2.22 . We extend binary operations to sets in the usual way, e.g. $a \wedge B=$ $\{a \wedge b: b \in B\}, A \cdot b=\{a \cdot b: a \in A\}$, etc... .

A lattice $L$ is join-infinite distributive (JID) if

$$
a \wedge(\bigvee B)=\bigvee(a \wedge B)
$$

whenever the supremum on the left hand side exists. The dual condition is called meet-infinite distributive (MID). Note that distributivity does not imply either of these properties, even for complete lattices (see e.g. [DP02]). Also note that every Boolean algebra is both join-infinite and meet-infinite distributive (see e.g. [Kop89, Ch. 1, §1]). We will need to recall that $a$ can be replaced with a supremum:

Proposition 2.23. If $L$ is (JID), then $(\bigvee A) \wedge(\bigvee B)=\bigvee(A \wedge B)$ whenever $\bigvee A$ and $\bigvee B$ both exist. Similarly for the (MID).

Definition 2.24. We shall call a monoid $(M, \cdot)$ infinitely left --distributive over $\vee$ or infinitely left $(\cdot, \mathrm{V})$-distributive if

$$
a \cdot \bigvee B=\bigvee(a \cdot B) \text { for all } a \in M \text { and } B \subseteq M,
$$

whenever $\bigvee B$ exists, and call it infinitely right $(\cdot, \vee)$-distributive if

$$
(\bigvee A) \cdot b=\bigvee(A \cdot b) \quad \text { for all } A \subseteq M \text { and } b \in M
$$

whenever $\bigvee A$ exists, and we call the monoid infinitely $(\cdot, \vee)$-distributive if it is both infinitely left and right $(\cdot, \vee)$-distributive. The definition of infinite $(\cdot, \wedge)$ distributivity is exactly analogous.

Proposition 2.25. If $(M, \cdot)$ is infinitely $(\cdot, \vee)$-distributive, then $(\bigvee A) \cdot(\bigvee B)=$ $\bigvee(A \cdot B)$ whenever $\bigvee A$ and $\bigvee B$ both exist; similarly for infinite $(\cdot, \wedge)$-distributivity. 
Proposition 2.26. Let $(M,+)$ be a commutative monoid. Then infinite left and right $(+, \vee)$-distributivity both coincide with $(+, \vee)$-distributivity. And infinite left and right $(+, \wedge)$-distributivity both coincide with $(+, \wedge)$-distributivity.

Example 2.27. The monoid $(\mathbb{N},+)$ is a lattice monoid, because $\leq_{(\mathbb{N},+)}$ agrees with the usual ordering of $\mathbb{N}$, which is a lattice with supremum max and infimum min. It is a commutative cancellative and distributive lattice monoid, that embeds into the Abelian group of integers $(\mathbb{Z},+)$. It is also a complete semilattice satisfying (JID), (MID) and infinite $(+, \mathrm{W})$-distributivity.

Example 2.28. Let $I$ be an index set, and consider the product monoid $\left(\mathbb{N}^{I},+\right)$, i.e. with coordinatewise addition. Since the quasi order associated with a product monoid is the corresponding product of the associated quasi orders, $\left(\mathbb{N}^{I},+\right)$ is a commutative lattice monoid with $x \leq_{\left(\mathbb{N}^{I},+\right)} y$ iff $x(i) \leq y(i)$ for all $i \in I$. It is a complete semilattice that is cancellative, (JID), (MID) and infinitely +-distributive over $\boldsymbol{~} \mathrm{h}$, because all of these properties are preserved under products, i.e. the suprema and infima of a product lattice are taken coordinatewise (see also theorem 2.31). It embeds into the generalized Baer-Specker group $\left(\mathbb{Z}^{I},+\right)$.

We shall start simplifying the notation by writing $\leq$ instead of $\leq_{(M, \cdot)}$ and $\leq_{(M,+)}$.

Lemma 2.29. Every cancellative monoid $(M, \cdot)$ is infinitely left $(\cdot, \vee)$-distributive.

Proof. Suppose that $a \in M$ and $\bigvee B$ exists. $a \cdot b \leq a \cdot \bigvee B$ for all $b \in B$ by proposition 2.5, and thus $a \cdot \bigvee B$ is an upper bound of $a \cdot B$. On the other hand, suppose that $r$ is an upper bound of $a \cdot B$. Then for all $b \in B, r \geq a \cdot b$ implies that there exists $c_{b} \in M$ with

$$
a \cdot b \cdot c_{b}=r .
$$

Then by the cancellative property, there exists $d \in M$ with $b \cdot c_{b}=d$ for all $b \in B$. Now since $d \geq \bigvee B, r=a \cdot d \geq a \cdot \bigvee B$ by proposition 2.5, completing the proof that $\bigvee(a \cdot B)=a \cdot \bigvee B$.

Corollary 2.30. Every commutative cancellative monoid $(M,+)$ is infinitely +distributive over $\vee$.

Proof. Lemma 2.29 and proposition 2.26.

Theorem 2.31. Every commutative cancellative semilattice monoid $(M,+)$ is both infinitely $(+, \vee)$-distributive and $(+, \wedge)$-distributive.

Proof. Infinite $(+, \vee)$-distributivity is by corollary 2.30. Suppose then that $\wedge B$ exists. Then $a+b \geq a+\bigwedge B$ for all $b \in B$, and thus $a+\bigwedge B$ is a lower bound for $a+B$. To prove that it is in fact the greatest lower bound, since $M$ is a semilattice it will suffice take a lower bound $r \geq a+\bigwedge B$ and show that $r=a+\bigwedge B$. Now since $r \geq a, r-a \in M$. Therefore, for all $b \in B, r \leq a+b$ implies that $r-a \leq(a+b)-a=b$ by proposition 2.17. Thus $r-a \leq \bigwedge B$, and hence $r=a+(r-a) \leq a+\bigwedge B$ as required. 


\section{Order embeddings With PREREgular RANGe}

The main result of this section (theorem 3.38) is that order embeddings between posets, with some regularity property imposed on their ranges, are continuous with respect to the Scott topology on these posets. The other major theme of this section appears in a series of results (lemma 3.84-corollary 3.98) on extending a given continuous homomorphism from a suitably 'dense' subset of some lattice to the entire lattice.

3.1. Preregularity. We introduce the notion of preregularity and observe (lemma 3.15) that convex subsets of lattices are preregular. We also prove (lemma 3.16) that every dense 'subgroup' of a lattice monoid is regular.

Notation 3.1. For a poset $(P, \leq)$ and a subposet $A \subseteq P$ we write $\bigvee^{A} B$ for the supremum of $B \subseteq A$ taken in the poset $(A, \leq)$, and similarly for infima.

Proposition 3.2. Suppose $(P, \leq)$ is a poset and $A \subseteq P$ is a subposet. Then for all $B \subseteq A$ : if $\bigvee^{A} B$ and $\bigvee^{P} B$ both exist then $\bigvee^{P} B \leq \bigvee^{A} B$; and if $\bigwedge^{A} B$ and $\bigwedge^{P} B$ both exist then $\bigwedge^{P} B \geq \bigwedge^{A} B$.

Definition 3.3. Let $(P, \leq)$ be a poset. We call a subset $A \subseteq P$ order closed if for every nonempty $\emptyset \neq B \subseteq A$ : if $p=\bigvee^{P} B$ exists in $(P, \leq)$ then $p \in A$; and if $q=\bigwedge^{P} B$ exists in $(P, \leq)$ then $q \in A$.

We will want to consider a weaker property than order closed, and also to separate upwards and downwards closedness.

Definition 3.4. Let us call a subset $A \subseteq P$ upwards boundedly order closed if for every nonempty $B \subseteq A$ that has an upper bound in $A$, if $p=\bigvee^{P} B$ exists then $p \in A$; and we call $A$ downwards boundedly order closed if for every nonempty $B \subseteq A$ with a lower bound in $A$, if $p=\bigwedge^{P} B$ exists then $p \in A$. And we call $A \subseteq P$ boundedly order closed if it is both upwards and downwards boundedly order closed.

We can separate the order closed property into upwards order closed and downwards order closed analogously.

Remark 3.5. Note that an upwards order closed subset should be distinguished from an upwards closed subset $A$ of $P$, which of course refers to a set $A$ satisfying $\uparrow A=A$ where $\uparrow A=\{p \in P: p \geq a$ for some $a \in A\}$. To avoid possible confusion we follow [AJ94] and call them upper sets. Analogous remarks are made for downwards closed subsets, which we call lower sets.

Definition 3.6. For a subposet $A$ of $(P, \leq$ ), the upwards (downwards) order closure of $A$ is the smallest upwards (downwards) order closed $Q \subseteq P$ containing $A$.

Note that the upwards (downwards) order closure always exists because the family of upwards (downwards) order closed sets is closed under intersections. Indeed, they have explicit descriptions.

Proposition 3.7. The upwards order closure of $A \subseteq P$ is given by

$$
\{\bigvee B: B \subseteq A \text { and the supremum exists }\} \text {, }
$$


and thus the downwards order closure has the dual description.

Proposition 3.8. For any $B \subseteq A \subseteq P$, if $\bigvee^{P} B$ exists and is an element of $A$, then $\bigvee^{A} B=\bigvee^{P} B$. Similarly for infima.

Hence, for example:

Proposition 3.9. If $A \subseteq P$ is order closed then for all $\emptyset \neq B \subseteq A, \bigvee^{A} B=\bigvee^{P} B$ if the supremum exists in $P$. Similarly for infima.

On the other hand, the supremum may exist in the subposet $A$ but not in $P$. This leads to the following definition (see e.g. KKop89, Ch. 1, §1]).

Definition 3.10. A subset $A \subseteq P$ is upwards regular if for all $B \subseteq A$, if $a=\bigvee^{A} B$ exists in $(A, \leq)$ then $\bigvee^{P} B=a$ in $(P, \leq)$, and it is downwards regular if for all $B \subseteq A$, if $b=\bigwedge^{A} B$ exists in $A$ then $\bigwedge^{P} B=b$. A subset is regular if it is both upwards and downwards regular.

However, we want to avoid the singularity of empty suprema and infima, and thus we instead define the following concept.

Definition 3.11. A subset $A \subseteq P$ is upwards preregular if for all nonempty $\emptyset \neq$ $B \subseteq A$ : if $a=\bigvee^{A} B$ exists then $\bigvee^{P} B=a$, and it is downwards preregular if for all $\emptyset \neq B \subseteq A$ : if $b=\bigwedge^{A} B$ exists then $\bigwedge^{P} B=b$. It is preregular if it is both upwards and downwards preregular.

We point out that preregularity is a transitive property.

Proposition 3.12. Let $(P, \leq)$ be a poset. If $A \subseteq P$ is preregular, and $B \subseteq A$ is preregular as a subset of the poset $(A, \leq)$, then $B$ is preregular as a subset of the poset $P$.

Lemma 3.13. Every boundedly order closed subset of a complete semilattice is preregular.

Proof. Given a complete semilattice $L$, let $A \subseteq L$ be boundedly order closed, and $\emptyset \neq B \subseteq A$. If $\bigvee^{A} B$ exists, then $B$ has an upper bound in $A$, and thus $a=\bigvee^{L} B$ exists, and $a \in A$. Now $\bigvee^{A} B=a$ by proposition 3.8. And if $\bigwedge^{A} B$ exists then $B$ has a lower bound in $A$, and $\bigwedge^{L} B$ exists because $B \neq \emptyset$; hence, we can conclude that $\bigwedge^{L} B=\bigwedge^{A} B$.

Definition 3.14. Let $(O, \leq)$ be a quasi order. We call $A \subseteq O$ convex if $p, q \in A$ implies $[p, q] \subseteq A$ where $[p, q]$ is the interval $\{r \in O: p \leq r \leq q\}$.

Lemma 3.15. A convex subset of any lattice is preregular.

Proof. Suppose $(L, \leq)$ is a lattice, $A \subseteq L$ is convex, and $\emptyset \neq B \subseteq A$, say with $b \in B$. Assume $a=\bigvee^{A} B$ exists. Then if $p \in L$ is an upper bound of $B$, so is $p \wedge a$. However, $[b, a] \subseteq A$ and thus $b \leq p \wedge a \leq a$ implies $p \wedge a \in A$, which entails that $p \geq a$ as needed. Same for the infimum. 
Lemma 3.16. Let $(M,+)$ be a cancellative commutative lattice monoid. Then every dense ( $c f$. definition 3.53) submonoid of $M$ that is closed under subtraction is regular.

Proof. Let $D \subseteq M$ be a dense submonoid closed under subtraction. Suppose $A \subseteq D$ and $a=\bigvee^{D} A$. Assume towards a contradiction that $a \neq \bigvee^{M} A$. Then there is an upper bound $b \in M$ of $A$ such that $a \not \leq b$, and thus $a \wedge b$ is an upper bound of $A$, and $a \wedge b<a$, which by proposition 2.8 means there is a $c \in M^{+}$with $(a \wedge b)+c=a$. By density, there exists $d \leq c$ in $D^{+}$. Now $a-c=a \wedge b$, and thus $a-d$ exists and $a-d \geq a \wedge b$ by proposition 2.16. Thus $a-d$ is an upper bound of $A$. But $a \in D$, and hence $a-d \in D$ because it is closed under subtraction. Since $a-d<a$ this is contrary to $a$ being the supremum in $D$, thereby completing the proof of upwards regularity.

Suppose $A^{\prime} \subseteq D$ and $a^{\prime}=\bigwedge^{D} A^{\prime}$. Assuming towards a contradiction that $a^{\prime}$ is not the infimum in $M$, there exists a lower bound $b^{\prime}>a^{\prime}$ of $A^{\prime}$. Writing $a^{\prime}+c^{\prime}=b^{\prime}$, there exists $d^{\prime} \leq c^{\prime} \in D^{+}$by density. But since $D$ is a subsemigroup, $a^{\prime}+d^{\prime} \in D$, and it is a lower bound of $A^{\prime}$ since $a^{\prime}+d^{\prime} \leq b^{\prime}$. This is contrary to $a^{\prime}$ being the infimum since $a^{\prime}<a^{\prime}+d^{\prime}$, thereby completing the proof of downwards regularity.

Corollary 3.17. Let $(M,+)$ be a cancellative commutative lattice monoid. Then every dense submonoid that is closed under subtraction forms a sublattice.

\subsection{Order reflecting homomorphisms.}

Notation 3.18 . For a relation $(S, \leq)$, we write $S^{0}$ for the set of all $\leq$-minimal elements of $S$, and $S^{+}=S \backslash S^{0}$.

Note that a lattice has at most one minimal element in which case it is the 0 of the lattice.

Definition 3.19. Let $(O, \leq)$ be a quasi order. An element of $O$ is an atom if it is a nonminimal element that cannot be split, where we say that $p$ can be split if there exists $q, r \leq p$ in $O^{+}$such that $q$ is incompatible with $r$, written $q \not \approx r$ (i.e. there is no common extension of $q$ and $r$ in $\left.O^{+}\right)$. Write $\operatorname{At}(O, \leq)$ for the collection of atoms of $O$. $O$ is atomless if $O^{+}$has no atoms, whereas $O$ is atomic if $\operatorname{At}(O, \leq)$ is dense in $O$.

A mapping $\sigma: O \rightarrow Q$ between two quasi orders is said to preserve (non)atoms if $\sigma(a)$ is a (non)atom whenever $a$ is a (non)atom.

Proposition 3.20. In a Boolean algebra $(B, \leq), a \in B$ is an atom iff $a \neq 0$ and there is no $0<b<a$.

Remark 3.21. In the literature (e.g. DP02]), sometimes the definition of an atom of a lattice is as in the characterization of proposition 3.20, and this may disagree with our definition. Our terminology better fits the English definition of the word "atom", and moreover agrees with its usual usage in set theoretic forcing.

Example 3.22. The atoms of a power set Boolean algebra $(\mathcal{P}(X), \cup, \cap, \backslash, \emptyset, X)$ are precisely the singletons. Moreover, the relative atoms of some interval $[a, b]=\{x \subseteq$ $X: a \subseteq x \subseteq b\}$ of $\mathcal{P}(X)$ are precisely elements of the form $a \cup\{\xi\}$ for some $\xi \in b \backslash a$. Thus any interval of a power set Boolean algebra is atomic. 
Example 3.23. The atoms of a product lattice of the form $\prod_{i \in I} \alpha_{i}$ where each $\alpha_{i}$ is an ordinal, are precisely the members of the form $\xi \cdot \chi_{i}$ for some $i \in I$ and $0 \neq \xi<\alpha_{i}$. Moreover, if $x \in \prod_{i \in I} \alpha_{i}$ and $Y \subseteq \prod_{i \in I} \alpha_{i}$ is directed, i.e. every two elements of $Y$ has a common upper bound in $Y$, then the union of intervals $\bigcup_{y \in Y}[x, y]$ is a sublattice. Its atoms are members of the form $x+\xi \cdot \chi_{i}$ for some $i \in I$ and $0 \neq \xi<\alpha_{i}$. These sublattices are thus atomic.

Example 3.24. The category algebra $\operatorname{Cat}(X)$ (cf. 4.3 ) of a Hausdorff space is atomless.

The following results, proposition 3.25 through corollary 3.29, are consequences of the fact (lemma 3.28) that simultaneously order preserving and reflecting maps between quasi orders come close to being isomorphisms onto their range. Indeed they are preisomorphisms onto their range according to the terminology of [AJ94.

Proposition 3.25. Let $(O, \leq)$ and $(Q, \lesssim)$ be quasi orders. If $\sigma: O \rightarrow Q$ is both order preserving and reflecting then $p<q$ iff $\sigma(p) \lesssim \sigma(q)$ for all $p, q \in O$.

Proposition 3.26. If $\sigma: O \rightarrow Q$ is an order preserving and reflecting map between two quasi orders then the set of minimal elements is mapped to the set of all relatively minimal elements of the range, i.e. $\sigma\left[O^{0}\right]=\operatorname{ran}(\sigma)^{0}$.

Proposition 3.27. If $\sigma: O \rightarrow Q$ is both order preserving and reflecting, then the image of $O^{+}$is the positive part of the suborder $\operatorname{ran}(\sigma)$, i.e. $\sigma\left[O^{+}\right]=\operatorname{ran}(\sigma)^{+}$.

We let $O /$ asym denote the antisymmetric quotient, i.e. the equivalence classes modulo $p \sim_{\text {asym }} q$ if $p \leq q$ and $q \leq p$. Recall that for any quasi order this yields a poset, where the ordering $[p] \leq[q]$ if $p \leq q$ is well defined since $\sim_{\text {asym }}$ is a congruence for the quasi order.

Lemma 3.28. Let $(O, \leq)$ and $(Q, \lesssim)$ be quasi orders. Suppose $\sigma$ is an order preserving and reflecting map between $(O, \leq)$ and $(Q, \lesssim)$. Then $\bar{\sigma}: O /$ asym $\rightarrow Q /$ asym is well defined by

and is an embedding.

$$
\bar{\sigma}([p])=[\sigma(p)]
$$

Proof. $\bar{\sigma}$ is well defined because $\sigma$ is order preserving. Since $O$ / asym is a poset, it remains to show that $\bar{\sigma}$ is order preserving and reflecting. But since $\sigma$ is, $[p] \leq[q]$ iff $p \leq q$ iff $\sigma(p) \leq \sigma(q)$ iff $\bar{\sigma}([p])=[\sigma(p)] \leq[\sigma(q)]=\bar{\sigma}([q])$.

Corollary 3.29. Let $\sigma: O \rightarrow Q$ be both order preserving and reflecting. Then the image of the atoms are the relative atoms of the image, i.e. $\sigma[\operatorname{At}(O)]=\operatorname{At}(\operatorname{ran}(\sigma))$.

Proof. By lemma 3.28, $\bar{\sigma}[\operatorname{At}(O /$ asym $)]=\operatorname{At}(\operatorname{ran}(\bar{\sigma}))$. The proof is completed by noting that the atoms of the antisymmetric quotient consist of the equivalence classes of atoms.

3.3. Continuity. We examine continuity phenomena for order homomorphisms. Then some consequences (proposition 3.45-corollary 3.52) are deduced relevant to computing the range of continuous homomorphisms. 
Recall that a subset $A$ of a quasi order is directed if it is nonempty and every two elements of $A$ have a common upper bound in $A$. In Domain Theory, the fundamental topology on a poset is the Scott topology whose closed sets consist of all lower sets that are closed under directed suprema (i.e. the suprema of directed subsets), cf. AJ94], Smy92]. The supremum of a directed set $A$ is normally written as $\bigsqcup A$.

Definition 3.30. We call a function $\sigma: P \rightarrow Q$ between two posets continuous if it is topologically continuous for the Scott topologies on $P$ and $Q$. It is cocontinuous if it is topologically continuous for the Scott topologies of the duals of the posets $P$ and $Q$. It is dually continuous if it is both continuous and cocontinuous.

Lemma 3.31. Let $\sigma: P \rightarrow Q$ be a function between two posets. Then the following are equivalent.

(a) $\sigma$ is continuous.

(b) The conjunction of:

(1) $\sigma$ is a homomorphism (i.e. order preserving),

(2) $\sigma$ preserves directed suprema, i.e. $\sigma(\bigsqcup A)=\bigsqcup_{a \in A} \sigma(a)$ whenever $A \subseteq P$ is directed and $\bigsqcup A$ exists.

Proof. See e.g. [AJ94].

Example 3.32. Of course, homomorphisms need not be continuous. Indeed, if $\mathcal{U}$ is a nonprincipal ultrafilter on $\mathbb{N}$ then $\sigma: \mathcal{P}(\mathbb{N}) \rightarrow \mathcal{P}(\mathbb{N})$ given by $\sigma(A)=\emptyset$ if $A \notin \mathcal{U}$ and $\sigma(B)=\mathbb{N}$ if $B \in \mathcal{U}$ is a Boolean algebra homomorphism, yet it is clearly noncontinuous.

The following characterization of preservation of nonempty suprema, when the domain is a semilattice, is well known.

Lemma 3.33. Let $S$ be a semilattice and $Q$ a poset. Then the following are equivalent for any $\sigma: S \rightarrow Q$.

(a) $\sigma$ is continuous and join-preserving.

(b) $\sigma$ preserves arbitrary nonempty suprema, i.e. $\sigma(\bigvee A)=\bigvee_{a \in A} \sigma(a)$ whenever $A \subseteq S$ is nonempty and $\bigvee A$ exists.

Proof. Assume that $\sigma: S \rightarrow Q$ is join-preserving and continuous with respect to the Scott topologies on $S$ and $Q$. Suppose $A \subseteq S$ is nonempty and $\bigvee A$ exists. Let $\mathcal{F}$ be the set of all nonempty finite subsets of $A$. Then $\{\bigvee F: F \in \mathcal{F}\}$ is a directed subset of $S$ with $\bigsqcup_{F \in \mathcal{F}} \bigvee F=\bigvee A$, and thus by lemma 3.31(配),

$$
\sigma(\bigvee A)=\sigma\left(\bigsqcup_{F \in \mathcal{F}} \bigvee F\right)=\bigsqcup_{F \in \mathcal{F}} \sigma(\bigvee F)=\bigsqcup_{F \in \mathcal{F}} \bigvee_{a \in F} \sigma(a)=\bigvee_{a \in A} \sigma(a)
$$

Conversely, if $\sigma$ preserves nonempty suprema then in particular it preserves directed suprema, and thus is continuous by lemma 3.31.

Corollary 3.34. A semilattice homomorphism is continuous iff it preserves nonempty suprema. 
Corollary 3.35. A meet semilattice homomorphism is cocontinuous iff it preserves nonempty infima.

Proof. By corollary 3.34 since the dual of a meet semilattice is a (join) semilattice.

Corollary 3.36. A lattice homomorphism is dually continuous iff it preserves both nonempty suprema and infima.

Remark 3.37. The essential notion for us is preservation of nonempty suprema, as in lemma 3.33 (b). We were tempted to simply define continuity as this property, but this seemed a bit artificial. Category theoretic continuity does not quite capture this notion either. In the category of partial orders, all colimits are coproducts, which are suprema. Thus a mapping is cocontinuous in the category theoretic sense iff it preserves arbitrary suprema, and not just nonempty ones.

However, all of our example posets are semilattices and as we shall see, the embeddings under consideration are all semilattice homomorphisms. Thus by corollary 3.34 , for our purposes the two notions coincide.

Theorem 3.38. Every order embedding between two posets with a preregular range preserves all nonempty suprema. In particular, it is continuous.

Proof. Let $(P, \leq)$ and $(Q, \lesssim)$ be two posets, and $\sigma: P \rightarrow Q$ an embedding with preregular range. Take $\emptyset \neq A \subseteq P$ such that $\bigvee A$ exists. Then $\sigma(\bigvee A)=\bigvee_{a \in A}^{\operatorname{ran}(\sigma)} \sigma(a)$ since $(P, \leq) \cong(\operatorname{ran}(\sigma), \lesssim)$ via $\sigma$. And by preregularity, $\bigvee_{a \in A}^{\operatorname{ran}(\sigma)} \sigma(a)=\bigvee_{a \in A} \sigma(a)$. This proves that $\sigma$ preserves nonempty suprema, and thus it is continuous by lemma 3.31 .

Corollary 3.39. Every order embedding between two posets with preregular range preserves nonempty infima. In particular, it is cocontinuous.

Proof. By theorem 3.38, since any order embedding is also an embedding between the dual posets.

Preserving all suprema amounts to preserving all nonempty suprema, and mapping the minimum element of the domain, if it has one, to the minimum of the codomain. We do not want to impose the requirement of preserving minimums in our discourse, but we will note the following.

Corollary 3.40. Every order embedding between two posets with regular range preserves arbitrary suprema and infima, and thus is continuous and cocontinuous in the category theoretic sense ( $c f$. remark [3.37).

As mentioned, we will focus on semilattices.

Corollary 3.41. Every order embedding between two semilattices with preregular range is a continuous semilattice embedding (and as such, preserves nonempty suprema).

Proof. Theorem 3.38 and lemma 3.33 . 
Corollary 3.42. Every order embedding between two lattices with preregular range is a dually continuous lattice embedding (and thus preserves nonempty suprema and infima).

Corollary 3.43. Every order embedding between two lattices with convex range is a dually continuous lattice embedding.

Proof. Lemma 3.15 and corollary 3.42 ,

Let us point out the obvious, that order isomorphisms are automatically continuous.

Proposition 3.44. Every order isomorphism between two posets is dually Scott continuous and both continuous and cocontinuous in the categorical sense.

The remainder of this section is concerned with obtaining regularity properties of the range of some homomorphism. Sometimes the computation of the range is trivial:

Proposition 3.45. Suppose that $(O, \leq)$ is a quasi order that has both a minimum and maximum element, say $a$ and $b$, respectively. If $\sigma: O \rightarrow Q$ is a quasi order homomorphism between $O$ and some quasi order $(Q, \lesssim)$ with convex range then $\operatorname{ran}(\sigma)=[\sigma(a), \sigma(b)]=\{q \in Q: \sigma(a) \lesssim q \lesssim \sigma(b)\}$.

Lemma 3.46. Every continuous join-preserving order embedding between a complete semilattice and a poset has upwards boundedly order closed range.

Proof. Let $\sigma: L \rightarrow P$ be as in the hypothesis. Suppose $\emptyset \neq A \subseteq \operatorname{ran}(\sigma)$ is bounded by $p \in \operatorname{ran}(\sigma)$ and $\bigvee^{P} A$ exists. Then since $\sigma^{-1}(p)$ is an upper bound of $\sigma^{-1}[A]$, $q=\bigvee \sigma^{-1}[A]$ exists. Thus $\bigvee^{P} A=\sigma(q) \in \operatorname{ran}(\sigma)$ as required, by lemma 3.33.

Let us also point out a version of the preceding lemma that holds for homomorphisms that are not necessarily embeddings.

Lemma 3.47. Every continuous lattice homomorphism from a complete semilattice into a lattice has upwards boundedly order closed range.

Proof. Letting $\sigma: L \rightarrow M$ be as in the hypothesis, suppose $\emptyset \neq A \subseteq \operatorname{ran}(\sigma)$ is bounded by $p \in \operatorname{ran}(\sigma)$ and $\bigvee A$ exists. Choose $c \in L$ such that $\sigma(c)=p$, and for each $a \in A$, choose $b_{a} \in L$ such that $\sigma\left(b_{a}\right)=a$. Since $L$ is a complete semilattice, we can let $r=\bigvee_{a \in A}\left(b_{a} \wedge c\right)$. Then $\sigma(r)=\bigvee_{a \in A} \sigma\left(b_{a} \wedge c\right)=\bigvee_{a \in A}\left(\sigma\left(b_{a}\right) \wedge p\right)=$ $\bigvee_{a \in A} \sigma\left(b_{a}\right)=\bigvee A$, as required.

Lemma 3.48. Every cocontinuous meet semilattice homomorphism between a complete semilattice and a poset has downwards order closed range.

Proof. Let $\sigma: L \rightarrow P$ be as in the hypothesis. Suppose $\emptyset \neq A \subseteq \operatorname{ran}(\sigma)$ and $\bigwedge A$ exists in $P$. Observe that $p=\bigwedge \sigma^{-1}[A]$ exists by as $L$ is a complete semilattice (cf. \$1.1). Hence $\sigma(p)=\bigwedge A$ by corollary 3.35 , and $\sigma(p) \in \operatorname{ran}(\sigma)$ as wanted.

Corollary 3.49. Every order embedding between a complete semilattice and a poset with preregular range, in fact has a boundedly order closed range. 
Proof. Every order embedding between two posets with preregular range preserves nonempty suprema and infima, and in particular is dually continuous, by theorem 3.38 and corollary 3.39 . Thus result follows immediately from lemmas 3.46 and 3.48 ,

Definition 3.50. A map $f: O \rightarrow Q$ between two quasi orders is said to preserve boundedness if whenever $A \subseteq O$ is bounded (cf. \$1.1), so is $f[A] \subseteq Q$. More generally, a partial function $f: O \rightarrow Q$ is said to preserve boundedness in $O$ if $f[A]$ is bounded whenever $A \subseteq \operatorname{dom}(f)$ is bounded in $O$. On the other hand, a function $f: O \rightarrow Q$ is said to preserve unboundedness if whenever $A \subseteq O$ is unbounded, so is $f[A] \subseteq Q$.

Example 3.51. The embedding $i: \omega \rightarrow \omega+1$ (with $i(n)=n$ ) does not preserve unboundedness.

Corollary 3.52. Every order embedding between a complete semilattice and a poset preserving unboundedness, and with preregular range, in fact has an order closed range.

Proof. Let $\sigma: L \rightarrow P$ be an order embedding preserving unboundedness with preregular range, where $L$ is a complete semilattice. Note that it is in fact established in the proof of corollary 3.49 that $\sigma$ has downwards order closed range. Now suppose $\emptyset \neq A \subseteq \operatorname{ran}(\sigma)$ and $\bigvee A$ exists in $P$. Since $A$ is bounded, so must be $\sigma^{-1}[A]$ by preservation of unboundedness. Therefore, $p=\bigvee \sigma^{-1}[A]$ exists. But now we have $\bigvee A=\sigma(p) \in \operatorname{ran}(\sigma)$ as required, by theorem 3.38 .

3.4. Bases of lattices. We turn our attention to various notions of density for subsets of lattices, with the goal of finding one suitable for extending order embeddings. The technical definition of strong interval predensity is introduced; and we prove (in lemma 3.71) that for a substantial class of commutative monoids, subsemigroups forming a basis are strongly preinterval dense. The motivation for the various definitions will become clear in the next section $\$ 3.5$.

Definition 3.53. A subset $D$ of a quasi order $(O, \leq)$ is dense if every $p \in O^{+}$has a $d \leq p$ in $D^{+}$.

Notation 3.54. For a quasi order $(O, \leq), Q \subseteq O$ and $p \in O$, we let $Q_{p}$ denote the down set $\{q \in Q: q \leq p\}$.

Definition 3.55. A subset $D$ of a poset $(P, \leq)$ is join dense if every $p \in P$ satisfies

$$
p=\bigvee D_{p} \text {. }
$$

Definition 3.56. A subset $D$ of a poset $P$ is called interval predense if every $p<q$ in $P$ has a $d \in D$ with $d \not \leq p$ and $d \leq q$.

Proposition 3.57. For any subset of a poset, join dense $\rightarrow$ interval predense $\rightarrow$ dense.

The following should be compared with the fact that for a Boolean algebra $(B, \leq)$, a subset $D \subseteq B$ is dense iff it is join dense (see e.g. [Kop89, Ch. 2, §4]). 
Lemma 3.58. Let $L$ be a meet semilattice. Then the following are equivalent for all $D \subseteq L$.

(a) $D$ is interval predense.

(b) $D$ is join dense.

Proof. By proposition [3.57, we need to prove (a) $\rightarrow($ b $)$. Fix $p \in L$, and suppose that $q$ is an upper bound of $D_{p}$. We need to show that $p \leq q$. Supposing to the contrary, $p \wedge q<p$ and thus there exists $d \not \leq p \wedge q$ in $D_{p}$. But this contradicts the fact that $p \wedge q$ is an upper bound of $D_{p}$.

The results of section $\$ 2.1$ are applied here to show that dense subsemigroups of complete semilattice monoids are join-dense.

Theorem 3.59. Let $(M,+)$ be a commutative cancellative complete semilattice monoid. Then every dense subsemigroup of $M$ is join-dense.

Proof. Suppose $D \subseteq M$ is a dense subsemigroup. Take $p \in M$. The supremum $q=\bigvee D_{p}$ exists because we are dealing with a complete semilattice, and obviously $q \leq p$; hence, there is an $r \in M$ such that $q+r=p$. It remains to show that $r=0$. Supposing to the contrary, by denseness there exists $s \in D^{+}$with $s \leq r$. By corollary 2.30 we can use infinite right $(+, \vee)$-distributivity, to obtain

$$
q+s=\bigvee\left(D_{p}+s\right) \text {. }
$$

However, $D_{p}+s \subseteq D$ since $D$ is a subsemigroup. Thus in fact $D_{p}+s \subseteq D_{p}$ because $d+s \leq q+s$ for all $d \in D_{p}$ by proposition 2.6, and $q+s \leq p$ by proposition 2.5. Therefore, $q+s \leq q$ by (3.4), which implies $s=0$ by cancellativity and lemma 2.9. However, $0 \notin D^{+}$(see remark 2.12) , a contradiction.

Definition 3.60. We call a subposet $A$ of a poset $(P, \leq)$ flat if there exists $p \in P$ such that $a \wedge b=p$ for all $a \neq b$ in $A$. A lattice $(L, \leq)$ is called flat-complete if $\bigvee A$ exists for every flat $A \subseteq L$.

Example 3.61. Since $(\mathbb{N}, \leq)$ is a chain, it is trivially flat-complete because a flat subset of chain has at most two elements. Thus for any index set $I,\left(\mathbb{N}^{I}, \leq\right)$ is flat-complete because flat-completeness is preserved under products.

The notion of being a complete semilattice is incomparable with being flatcomplete.

Example 3.62. We consider two sublattices of the complete semilattice $[0, \infty)^{\mathbb{N}}$ with the product order, which is also flat-complete being a product of chains. The sublattice $(\mathbb{Q} \cap[0, \infty))^{\mathbb{N}}$ is not a complete semilattice since the nonnegative rationals form a lattice that is not a complete semilattice, but it is flat-complete. On the other hand the family of $B\left([0, \infty)^{\mathbb{N}}\right)$ of bounded sequences of nonnegative reals is clearly a complete semilattice, but not flat-complete.

However, in the case of Boolean algebras the following is well known (and easily proved).

Proposition 3.63. Every flat-complete Boolean algebra is a complete Boolean algebra. 
Definition 3.64. Let $(L, \leq)$ be a pointed lattice (cf. \$1.1). A basis for $L$ is a meet subsemilattice $B \subseteq L$ such that every $a \in L$ has a family $A \subseteq B$ satisfying

(i) $A$ is pairwise incompatible, i.e. $b \wedge c=0$ for all $b \neq c$ in $A$,

(ii) $\bigvee A=a$.

Remark 3.65. In set theoretic terminology pairwise incompatible families are called antichains; however, this disagrees with its usage elsewhere, as a pairwise incomparable set (e.g. [DP02, , Eng97]).

Lemma 3.66. Every dense meet subsemilattice of a complete Boolean algebra is basis.

Proof. Let $D$ be dense. Fixing $a \in D$, let $A \subseteq D$ be a maximal pairwise incompatible family below $a$. Then $\bigvee A$ exists by completeness, and we cannot have $\bigvee A<a$ or else there exists $b \leq a-\bigvee A$ in $D^{+}$, and thus $b \notin A$ contradicting its maximality.

Example 3.67. Clopen sets are in particular regular open sets. Thus the collection of equivalence classes of the clopen subsets of $X$ forms a sublattice of Cat $(X)$. Let $X$ be a zero dimensional topological space. Then the family of clopen sets identifies with a dense subset of $\operatorname{Cat}(X)$, and therefore it forms a basis by lemma 3.66 .

Definition 3.68. We will say that a subset $D$ of a lattice $(L, \leq)$ is strongly interval predense if for every $p<q$ in $L$ there exists $d \in D$ such that

(i) $d \leq q$,

(ii) $d \wedge p<d$,

(iii) $d \wedge p \in D$.

This is equivalent to adding condition (iii) to interval predensity.

Being join-dense does not entail being strongly interval predense.

Example 3.69. Let $\Theta$ be some initial segment of the ordinals with its usual linear ordering $\in$. Then $(\Theta, \in)$ is a complete semilattice, and is moreover a complete lattice when it is closed (e.g. $\Theta=\omega+1$ ). Let $S_{\Theta}$ be the set of all successor ordinals in $\Theta$. Then $S_{\Theta}$ is evidently join-dense and thus interval predense, but so long as $\omega+1 \in \Theta$, it is not strongly interval predense, because $\omega<\omega+1$ but there is no successor ordinal satisfying both (iii) and (iii) for $p=\omega$.

Strong interval predensity does not entail preregularity.

Example 3.70. Consider the complete Boolean algebra $(\mathcal{P}(X), \subseteq)$ where $X$ is some infinite set. Then $\operatorname{Fin}(X)$, the set of all finite subsets of $X$ forms a sublattice, and thus $\operatorname{Fin}(X) \cup\{X\}$ is a bounded sublattice. It is strongly interval predense, because for any $a \subset b$ in $\mathcal{P}(X)$, picking $x \in b \backslash a,\{x\} \in \operatorname{Fin}(X) \cup\{X\},\{x\} \subseteq b$ and $\{x\} \cap a=\emptyset \subset\{x\}$ is in $\operatorname{Fin}(X)$. However, $\operatorname{Fin}(X) \cup\{X\}$ is not preregular, because taking any infinite $y \subseteq X, \bigvee^{\operatorname{Fin}(X) \cup\{X\}} \operatorname{Fin}(y)=X$ whereas $\bigvee^{\mathcal{P}(X)} \operatorname{Fin}(y)=y$.

Lemma 3.71. Let $(M,+)$ be a commutative cancellative complete semilattice monoid that is moreover (JID). Then every subsemigroup that is a basis is also strongly interval predense. 
Proof. Suppose that $B$ is a subsemigroup that is a basis. Take $a<b$ in $M$, and let $c \in M$ satisfy $a+c=b$. Hence $c \neq 0$. Since $B$ is a basis there is a pairwise incompatible family $A \subseteq B$ with supremum $a$. Fix any $d \in A$. First we consider the case $c \wedge d=0$. Since $B$ is dense there is a $\bar{d} \in B_{c}^{+}$. Then $d+\bar{d} \in B$ because $B$ is a subsemigroup. And theorem 2.31 implies that $M$ is $(+, \mathrm{W})$-distributive, and hence $d+\bar{d}=d \vee \bar{d}$ by lemma 2.20. Now $d+\bar{d}$ witnesses that $B$ is strongly interval predense because $d+\bar{d} \leq a+c=b,(d+\bar{d}) \wedge a=(d \vee \bar{d}) \wedge a=(d \wedge a) \vee(\bar{d} \wedge a)=d \in B$ by distributivity, and $d<d+\bar{d}$.

Otherwise, when $c \wedge d \neq 0$, we choose $\bar{d} \in B_{c \wedge d}^{+}$. Then $d+\bar{d} \in B, d+\bar{d} \leq a+c=b$, and using (JID), $(d+\bar{d}) \wedge a=(d+\bar{d}) \wedge \bigvee A=\bigvee_{p \in A}(d+\bar{d}) \wedge p=(d+\bar{d}) \wedge d=d$ by lemma 2.21, as required.

3.5. Extensions of embeddings. In this section we are concerned with extending some homomorphism from a join-dense subset of a lattice to the entire lattice, while preserving additional properties. Two of the main results are theorem 3.92 which allows for the extension of a lattice embedding of a strongly interval predense basis to a continuous lattice embedding of the entire lattice, and theorem 3.96 which allows us to do the same while also maintaining a convex range. We also introduce the notion of the $P$-Scott topology on a subposet of some poset $(P, \leq)$.

3.5.1. The subposet topology. At this juncture we need to look more closely at the topology on a subposet $Q$ of $(P, \leq)$. The point is that the Scott topology on $Q$ as given by its ordering $\leq$, may differ from the subspace topology that $Q$ inherits from the Scott topology on $P$, and moreover the two topologies may be incomparable. In fact, we are interested in a third topology on $Q$.

Definition 3.72. Let $(P, \leq)$ be a poset and $Q \subseteq P$. We say that $A \subseteq Q$ is $P$ closed under directed suprema if for every directed $A \subseteq Q$, if $a=\bigsqcup^{P} A$ exists and is in $Q$, then $a \in A$. Similarly, $A \subseteq Q$ is said to be upwards $P$-order closed if: for all $\emptyset \neq B \subseteq A$, if $a=\bigvee^{P} B$ exists and is in $Q$ then $a \in A$. The dual notions are called $P$-closed under downwards directed infima and downwards $P$-order closed, respectively.

Proposition 3.73. If $F \subseteq P \supseteq Q$ is closed under directed suprema (downwards directed infima), then $F \cap Q$ is $P$-closed under directed suprema (downwards directed infima).

Proposition 3.74. If $F \subseteq P \supseteq Q$ is upwards (downwards) order closed in $P$, then $F \cap Q$ is upwards (downwards) P-order closed as a subposet of $(Q, \leq)$.

Proposition 3.75. If $A \subseteq Q \subseteq P$ is closed under directed suprema (downwards directed infima) in the poset $(Q, \leq)$, then it is also $P$-closed under directed suprema (downwards directed infima).

Proof. By proposition 3.8.

Proposition 3.76. If $A \subseteq Q \subseteq P$ is upwards (downwards) order closed as a subposet of $(Q, \leq)$, then it is also upwards (downwards) P-order closed. 
Proof. By proposition 3.8 .

Notice that if $Q$ is a preregular subset of $P$, then $P$-closed coincides with closed. Thus, e.g.

Proposition 3.77. Let $Q$ be a preregular subset of $P$. Then $A \subseteq Q$ is closed under directed suprema iff it is $P$-closed under directed suprema.

Definition 3.78. Let $(P, \leq)$ be a poset and $Q \subseteq P$. Then the family of complements of lower subsets of $Q$ that are $P$-closed under directed suprema is a topology on $Q$; we call it the $P$-Scott topology on $Q$. A function $\sigma: Q \rightarrow R$, where $R$ is some poset, is called $P$-continuous if it is a continuous function with respect to the $P$-Scott topology on $Q$ and the Scott topology on $R$.

Proposition 3.79. The $P$-Scott topology on a subposet $Q \subseteq P$ is a common refinement of the its Scott topology and its subspace topology.

Proof. The P-Scott topology refines the Scott topology by proposition 3.75, and it refines the subspace topology by proposition 3.73 .

Note that for $Q=P$, the $P$-Scott topology is just the Scott topology. More generally:

Proposition 3.80. If $Q \subseteq P$ is preregular then the Scott topology on $Q$ coincides with its P-Scott topology.

Proof. By proposition 3.77

Corresponding to lemma 3.31 we have:

Proposition 3.81. Let $Q$ be a subposet of $(P, \leq)$, and $(R, \lesssim)$ some poset. Then any function $\sigma: Q \rightarrow R$ is $P$-continuous iff it is a homomorphism that preserves directed suprema in $P$, i.e. $\sigma\left(\bigsqcup^{P} A\right)=\bigsqcup_{a \in A} \sigma(a)$ whenever $A \subseteq Q$ is directed and $\bigsqcup^{P} A$ exists and is in $Q$.

And corresponding to lemma 3.33,

Proposition 3.82. Suppose $(S, \leq)$ is a semilattice and $Q \subseteq S$ is a subsemilattice, and suppose $(R, \lesssim)$ is a poset. Then any function $\sigma: Q \rightarrow R$ is $S$-continuous and join-preserving iff it preserves nonempty suprema in $P$, i.e. $\sigma\left(\bigvee^{P} A\right)=\bigvee_{a \in A} \sigma(a)$ whenever $A \subseteq Q$ and $\bigvee^{P} A$ exists and is in $Q$.

The following lemma shows that for complete semilattices, convexity of the range of an embedding follows from its convexity when suitably restricted to an interval predense subset of its domain.

Lemma 3.83. Let $L$ and $M$ be complete semilattices, with $M$ satisfying the (JID), and let $\sigma: L \rightarrow M$ be a continuous semilattice embedding. Suppose $0 \in D \subseteq L$ and $E \subseteq M$ are both join-dense (equivalently, interval predense), $E$ is a subsemilattice, and that $\sigma[D] \subseteq E$ is a convex subset of $E$. Then $\operatorname{ran}(\sigma)$ is convex. 
Proof. Given $p \leq q$ in $\operatorname{ran}(\sigma)$, we choose $p \leq r \leq q$ and prove that $r \in \operatorname{ran}(\sigma)$. Let

$$
s=\bigvee\{c \in \sigma[D]: c \leq r\},
$$

and note that since $0 \in D, p \in \operatorname{ran}(\sigma)$ implies that the set on the right is nonempty, namely $\sigma(0) \leq p \leq r$ is in $\sigma[D]$. Observe that $s \in \operatorname{ran}(\sigma)$ because $\operatorname{ran}(\sigma)$ is upwards boundedly order closed by lemma 3.46. Therefore it suffices to prove $r=s$.

Suppose to the contrary that $s<r$. Since $D$ is join-dense, $\sigma^{-1}(q)=\bigvee\{d \in D$ : $\left.d \leq \sigma^{-1}(q)\right\}$, and thus by corollary [3.34, $q=\bigvee\{c \in \sigma[D]: c \leq q\}$. Now by the (JID),

$$
r=r \wedge q=\bigvee\{r \wedge c: c \in \sigma[D], c \leq q\} .
$$

Hence there exists $c \in \sigma[D]$ such that $s \wedge c=s \wedge(r \wedge c)<r \wedge c$. Since $E$ is interval predense, there exists $d \in E$ such that $d \leq r \wedge c$ but

$$
d \not \leq s \wedge c .
$$

We now have $d^{\prime}=d \vee \sigma(0) \in E$ since $E$ is a subsemilattice, and thus as $\sigma(0) \leq d^{\prime} \leq c$, $d^{\prime} \in \sigma[D]$ by convexity. But then (3.5) says that $d^{\prime} \leq s$, which would imply $d \leq s \wedge c$, contradicting (3.7).

Preservation of boundedness (cf. definition 3.50) allows one to extend continuous order homomorphisms from join-dense subsets. However, embeddings do not necessarily extend. For example consider the continuous partial embedding $j: \omega+2-\rightarrow$ $\omega+1$ where $j(n)=n$ for $n<\omega$ and $j(\omega+1)=\omega$; the unique extension to a continuous homomorphism on $\omega+2$ maps $\omega$ to $\omega$ and thus is not an injection.

Lemma 3.84. Let $L$ be a (JID) lattice, and let $M$ be a complete semilattice. Suppose $D \subseteq L$ is a join-dense meet subsemilattice. Then every function $\sigma: D \rightarrow M$ that preserves nonempty suprema in $L$ (cf. proposition 3.82), and preserves boundedness in $L$, has an extension to a continuous semilattice homomorphism on $L$, that is uniquely determined on $L^{+}$. And this extension is moreover a lattice homomorphism when $\sigma$ is meet-preserving and $M$ is (JID).

Proof. Let $\sigma: D \rightarrow M$ be as specified in the hypothesis. We can define $\bar{\sigma}: L \rightarrow M$ by

$$
\bar{\sigma}(p)=\bigvee_{d \in D_{p}} \sigma(d)
$$

because the supremum exists as $\sigma\left[D_{p}\right]$ is bounded. $\bar{\sigma}(d)=\sigma(d)$ for all $d \in D$ because $\sigma$ is order preserving, and it is also clear that $\bar{\sigma}$ is order preserving. Suppose $\emptyset \neq A \subseteq$ $L$ and $\bigvee A$ (i.e. $\bigvee^{L} A$ ) exists. Clearly $\bigvee_{p \in A} \bar{\sigma}(p) \leq \bar{\sigma}(\bigvee A)$; while every $d \in D \bigvee A$ satisfies $\bigvee_{p \in A}^{L}\left(d \wedge D_{p}\right)=d \wedge \bigvee_{p \in A}^{L} D_{p}=d \wedge \bigvee A=d$ since $L$ is (JID) and $D$ is joindense, and thus $\bigvee_{p \in A} \bar{\sigma}(p)=\bigvee_{p \in A} \bigvee_{d^{\prime} \in D_{p}} \sigma\left(d^{\prime}\right) \geq \bigvee_{p \in A} \bigvee_{d^{\prime} \in D_{p}} \sigma\left(d \wedge d^{\prime}\right)=\sigma(d)$ since $D$ is a meet subsemilattice and since $\sigma$ preserves nonempty suprema in $L$. This proves the other inequality, establishing that $\bar{\sigma}$ preserves nonempty suprema. Thus $\bar{\sigma}$ is a continuous semilattice homomorphism by corollary 3.34 . 
Claim 3.85. If $\sigma$ is meet-preserving and $M$ is (JID) then $\bar{\sigma}$ is meet-preserving, and thus is a lattice homomorphism.

Proof. Using the fact that $D_{p \wedge q}=D_{p} \wedge D_{q}$ since $D$ is a meet subsemilattice for the second equality, and the fact that $\sigma$ is meet-preserving and proposition 2.23 for the third,

$$
\begin{aligned}
\bar{\sigma}(p \wedge q) & =\bigvee_{d \in D_{p \wedge q}} \sigma(d) \\
& =\bigvee_{c \in D_{p}} \bigvee_{d \in D_{q}} \sigma(c \wedge d) \\
& =\left(\bigvee_{c \in D_{p}} \sigma(c)\right) \wedge\left(\bigvee_{d \in D_{q}} \sigma(d)\right) \\
& =\bar{\sigma}(p) \wedge \bar{\sigma}(q) .
\end{aligned}
$$

For all $p \in L$, if $D_{p} \neq \emptyset$ then equation (3.8) must hold for any continuous extension of $\sigma$ to a semilattice homomorphism, proving uniqueness on $L^{+}$.

Remark 3.86. We do not necessarily have a unique extension to a pointed lattice $L$ unless 0 is already in $D$. For example, $\mathbb{N}^{+}=\{1,2, \ldots\}$ is join-dense in $\mathbb{N}$, but $n \mapsto n+1$ has two different extensions to a continuous lattice homomorphism. Of course, this freedom at 0 disappears if we require that all suprema are preserved (i.e. category theoretic continuity).

In our intended applications, we are extending from a join-dense sublattice, and we can then state the preceding result in the following simplified form.

Corollary 3.87. Let $L$ be a (JID) lattice, and $M$ be a complete semilattice. Suppose $D \subseteq L$ is a join-dense sublattice. Then every L-continuous semilattice homomorphism $\sigma: D \rightarrow M$, that preserves boundedness in $L$, has an extension to a continuous semilattice homomorphism on L, uniquely determined on $L^{+}$. This extension is moreover a lattice homomorphism when $\sigma$ is a lattice homomorphism and $M$ is (JID).

Proof. By lemma 3.84 and proposition 3.82 .

We use flat-completeness to ensure preservation of boundedness.

Lemma 3.88. Let $L$ be a lattice that is (JID), and let $M$ be a flat-complete lattice. Suppose $B \subseteq L$ is a basis. Then every meet-preserving order homomorphism between $B$ and $M$, that preserves nonempty suprema in $L$, preserves boundedness in $L$.

Proof. Let $\sigma: B \rightarrow M$ be as specified by the hypothesis. Given $0 \neq p \in L$ we must show that $\sigma\left[B_{p}\right]$ is bounded in $M$. Find a pairwise incompatible $A \subseteq B_{p}$ such that $\bigvee A=p$. Since $\sigma$ is meet-preserving, $\sigma(a) \wedge \sigma(b)=\sigma(0)$ for all $a \neq b$ in $A$, and thus $\sigma[A]$ is flat. Therefore $\bigvee_{a \in A} \sigma(a)$ exists by flat-completeness. It now suffices to prove that $\bigvee_{a \in B_{p}} \sigma(a)=\bigvee_{a \in A} \sigma(a)$. But for any $c \in B_{p}, c=c \wedge p=\bigvee_{a \in A}^{L} c \wedge a$ by the (JID), and thus as $A \neq \emptyset$, by preservation of nonempty suprema in $L$, $\sigma(c)=\bigvee_{a \in A} \sigma(c \wedge a) \leq \bigvee_{a \in A} \sigma(a)$, as wanted. 
Combining corollary 3.87 with the preceding lemma, we obtain the following result.

Corollary 3.89. Let $L$ be a lattice, and $M$ be a complete and flat-complete semilattice, with both (JID). Suppose $B \subseteq L$ is a sublattice that forms a basis. Then every $L$-continuous lattice homomorphism $\sigma: B \rightarrow M$ has an extension to a continuous lattice homomorphism on $L$, unique on $L^{+}$.

Proof. Note that bases are obviously join-dense.

Lattice homomorphisms with codomain a preregular subset of $M$ can also be extended.

Corollary 3.90. Let $L$ be a lattice, and $M$ be a complete and flat-complete semilattice, with both (JID). Suppose $B \subseteq L$ is a sublattice forming a basis, and $E \subseteq M$ is a preregular subset of $M$. Then every $L$-continuous order homomorphism $\sigma: B \rightarrow E$ that is both join and meet-preserving has an extension to a continuous lattice homomorphism $\bar{\sigma}: L \rightarrow M$, unique on $L^{+}$.

Proof. Letting $\sigma^{\prime}: B \rightarrow M$ be the same function as $\sigma$ but with codomain $M$, $\sigma^{\prime}$ is a lattice homomorphism because $E$ is preregular. And by propositions 3.79 and 3.80, the Scott topology on $E$ refines its subspace topology, and thus $\sigma^{\prime}$ is also $L$-continuous. Now the result is immediate from corollary 3.89 .

The notion of a strongly interval predense subset (cf. definition 3.68) was introduced to allow boundedness preserving embeddings to be extended.

Corollary 3.91. Let $L$ be a lattice and $M$ be a complete semilattice with both satisfying the (JID). Suppose $D \subseteq L$ is a strongly interval predense meet subsemilattice. Then every meet subsemilattice embedding of $D$ into $M$ that preserves nonempty suprema in $L$, and that preserves boundedness in $L$, has an extension to a continuous lattice embedding of $L$ into $M$, uniquely determined on $L^{+}$.

Proof. $D$ is join-dense by lemma 3.58 . Hence by lemma 3.84, there is a continuous lattice homomorphism $\bar{\sigma}: L \rightarrow M$ extending $\sigma$, uniquely determined by equation (3.8) on $L^{+}$. It remains to verify that $\bar{\sigma}$ is an embedding.

By proposition 1.1. it suffices to show that $\bar{\sigma}$ is strictly order preserving. Take $p<q$ in $L$, and suppose towards a contradiction that $\bar{\sigma}(p)=\bar{\sigma}(q)$. Since $D$ is strongly interval predense, there exists $d \in D_{q}$ such that $d \wedge p<d$ and $d \wedge p \in D$. But then since $\sigma$ is an embedding, we have

$$
\begin{aligned}
\bar{\sigma}(d \wedge p) & =\sigma(d \wedge p) \\
& <\sigma(d) .
\end{aligned}
$$

However, by assumption, $\bar{\sigma}(d \wedge p)=\bar{\sigma}(d) \wedge \bar{\sigma}(p)=\sigma(d) \wedge \bar{\sigma}(q)=\sigma(d)$, contradicting (3.10).

Now we come to the main result of this section, that embeddings into a complete flat-complete semilattice can be extended from a strongly interval predense basis. 
Theorem 3.92. Let $L$ be a lattice and $M$ a complete semilattice that is flat-complete, with both (JID). Suppose $B \subseteq L$ is a strongly interval predense basis. Then every meet subsemilattice embedding of $B$ into $M$ that preserves nonempty suprema in $L$, has an extension to a continuous lattice embedding of $L$ into $M$, unique on $L^{+}$.

Proof. Let $\sigma: B \rightarrow M$ be as hypothesized. Then $\sigma$ preserves boundedness in $L$ by lemma 3.88. The result now follows from corollary 3.91 .

We obtain a very natural simplified form when $B$ is a sublattice.

Corollary 3.93. Let $L$ be a lattice and $M$ a complete semilattice that is flatcomplete, with both (JID). Suppose $B \subseteq L$ is a sublattice that forms a strongly interval predense basis. Then every $L$-continuous lattice embedding of $B$ into $M$ has an extension to a continuous embedding of $L$ into $M$, unique on $L^{+}$.

Proof. By theorem 3.92 and proposition 3.82 .

Embeddings with preregular range satisfy the hypothesis of the main theorem.

Corollary 3.94. Let $L$ be a (JID) lattice, and let $M$ be a (JID) complete semilattice that is also flat-complete. Suppose $B \subseteq L$ is a strongly interval predense basis. Then every order embedding of $B$ into $M$ with preregular range has an extension to a continuous lattice embedding of $L$ into $M$, unique on $L^{+}$.

Proof. Let $\sigma: B \rightarrow M$ be an order embedding with convex range. By theorem 3.38 , $\sigma$ preserves arbitrary nonempty suprema, and thus in particular preserves nonempty suprema in $L$. And corollary 3.39 implies that $\sigma$ is meet-preserving. Therefore $\sigma$ extends to a continuous lattice embedding of $L$ into $M$, uniquely on $L^{+}$, by theorem 3.92 .

We shall want to extend an embedding from a subsemigroup forming a basis.

Corollary 3.95. Let $(L,+)$ be a commutative cancellative (JID) complete semilattice monoid, and let $M$ be a (JID) complete flat-complete semilattice. Suppose $B \subseteq L$ is a subsemigroup that forms a basis. Then every meet subsemilattice embedding of $B$ into $M$ preserving nonempty suprema in $L$, has an extension to a continuous lattice embedding of $L$ into $M$, unique on $L^{+}$.

Proof. By theorem 3.92, because $B$ is strongly interval predense by lemma 3.71 .

The following result allows one to extend a continuous embedding from a basis of a complete semilattice to the whole semilattice while maintaining a convex range.

Theorem 3.96. Let $L$ and $M$ be complete semilattices that are (JID), with $M$ flatcomplete. Suppose $0 \in B \subseteq L$ is a strongly interval predense basis and $E \subseteq M$ is a join-dense preregular sublattice. Then every order embedding of $B$ into $E$ with convex range (in $E$ ) has a unique extension to a continuous lattice embedding $\sigma: L \rightarrow M$, and moreover $\operatorname{ran}(\sigma)$ is convex.

Proof. Let $\sigma: B \rightarrow E$ be an order embedding with convex range. Since $E$ is a lattice, $\operatorname{ran}(\sigma)$ is preregular in $E$ by lemma 3.15. And thus by proposition 3.12, $\operatorname{ran}(\sigma)$ is a preregular subset of $M$. Now applying corollary 3.94, $\sigma$ has an extension, say $\bar{\sigma}$ : 
$L \rightarrow M$, to a continuous lattice embedding of $L$ into $M$, uniquely determined on $L^{+}$. Hence $\bar{\sigma}$ is in fact the unique extension of $\sigma$ to a continuous lattice homomorphism by remark 3.86. And as the hypotheses of lemma 3.83 are all satisfied, $\operatorname{ran}(\bar{\sigma})$ is convex.

Corollary 3.97. Let $(L,+)$ be a commutative cancellative complete semilattice monoid, and let $M$ be a complete flat-complete semilattice, with both (JID). Suppose $B \subseteq L$ is a submonoid forming a basis and $E \subseteq M$ is a join-dense preregular sublattice. Then every order embedding $\sigma: B \rightarrow E$ with convex range has a unique extension to a continuous lattice embedding of $L$ into $M$ with convex range.

Proof. Theorem 3.96 and lemma 3.71 .

Our intended application uses the following corollary.

Corollary 3.98. Let $(L,+)$ be a commutative cancellative complete semilattice monoid, and let $(M,+)$ be a commutative cancellative complete and flat-complete semilattice monoid, with both (JID). Suppose $B \subseteq L$ is a submonoid forming a basis and $E \subseteq M$ is a dense submonoid closed under subtraction. Then every order embedding $\sigma: B \rightarrow E$ with convex range has a unique extension to a continuous lattice embedding of $L$ into $M$ with convex range.

Proof. By lemma 3.16, $E$ is regular and in particular preregular, and thus is also a sublattice (corollary 3.17). Now the result is an immediate consequence of corollary 3.97.

\section{ThE PARTIAL ORDERS}

A precise description is obtained of the partial order embeddings with convex range within the following classes of posets: power set algebras; powers of $\mathbb{N}$; category algebras; Baire functions with codomain a power of $\mathbb{N}$, modulo almost always equality; and continuous functions with codomain a power of $\mathbb{N}$, in subsections 4.14.5, respectively. In $\$ 4.6$, these embeddings are discussed for the following classes: Baire functions with codomain a power set algebra, modulo almost always equality; measurable functions with respect to some measure space, modulo almost everywhere equality; the Boolean algebra $\mathcal{P}(\mathbb{N}) /$ Fin; and the lattice quotient $\mathbb{N}^{\mathbb{N}} /$ Fin of the irrationals ordered by eventual dominance.

Embeddings with convex range are considered exclusively. The results here fail otherwise as demonstrated in example 4.5.

4.1. Power set algebras. We begin by considering order embeddings between power set algebras.

Theorem 4.1. Order embeddings $\sigma$ from $(\mathcal{P}(X), \subseteq)$ into $(\mathcal{P}(Y), \subseteq)$ with convex range consist precisely of maps of the form

$$
\sigma(a)=h[a] \cup b \text { for all } a \subseteq X,
$$

for some injection $h: X \rightarrow Y$ and some $b \subseteq Y$ with $h[X] \cap b=\emptyset$. All of these maps are moreover dually continuous lattice embeddings. Furthermore, $h$ is a bijection iff $\sigma$ is a Boolean algebra isomorphism between $\mathcal{P}(X)$ and $\mathcal{P}(Y)$. 
Proof. We have $\operatorname{ran}(\sigma)=[\sigma(\emptyset), \sigma(X)]$ by proposition 3.45, Thus for every singleton, $\sigma(\{x\})=\sigma(\emptyset) \cup\left\{y_{x}\right\}$ for some $y_{x} \in \sigma(X) \backslash \sigma(\emptyset)$, by corollary 3.29 and example 3.22. By corollary 3.43, $\sigma$ is dually continuous. In particular, continuity implies by lemma 3.33 that $\sigma(a)=\sigma(\emptyset) \cup\left\{y_{x}: x \in a\right\}$ for all $a \subseteq X$. Thus the desired $h$ is defined by

$$
h(x)=y_{x},
$$

with $b=\sigma(\emptyset)$. Conversely, any $\sigma$ of the above form is easily seen to be an embedding whenever $h$ is an injection and $h[X] \cap b=\emptyset$. In the case where $h$ is a bijection, $h[X]=Y$ implies $b=\emptyset$ as $h[X] \cap b=\emptyset$, and thus $\operatorname{ran}(\sigma)=\mathcal{P}(Y)$ implies $\sigma$ is moreover an isomorphism.

Corollary 4.2. Every map $\sigma: \mathcal{P}(X) \rightarrow \mathcal{P}(Y)$ satisfying:

(a) $a \subseteq b$ iff $\sigma(a) \subseteq \sigma(b)$, for all $a, b \subseteq X$,

(b) $\sigma(a) \subseteq c \subseteq \sigma(b)$ implies $c \in \operatorname{ran}(\sigma)$, for all $a, b \subseteq X$ and $c \subseteq Y$,

is of the form

$$
\sigma(a)=h[a] \cup \sigma(\emptyset) \text { for all } a \subseteq X,
$$

for some injective $h: X \rightarrow Y$.

Proof. Theorem 4.1 is applied to the posets $(\mathcal{P}(X), \subseteq)$ and $(\mathcal{P}(Y), \subseteq)$, as conditions (国) and (b) translate to $\sigma$ being an embedding with convex range.

Corollary 4.3. The range of any embedding $\sigma: \mathcal{P}(X) \rightarrow \mathcal{P}(Y)$ with convex range is the interval Boolean algebra $[\sigma(\emptyset), \sigma(X)]$, and $i^{-1} \circ \sigma$ is in fact a Boolean algebra isomorphism, where $i^{-1}$ any left inverse of the inclusion function $i:[\sigma(\emptyset), \sigma(X)] \rightarrow$ $\mathcal{P}(Y)$.

The following result is well known, e.g. it is mentioned in [She98, pp. 171].

Corollary 4.4. Every quasi order isomorphism $\sigma: \mathcal{P}(X) \rightarrow \mathcal{P}(Y)$ is of the form $\sigma(a)=h[a]$ where $h: X \rightarrow Y$ is a bijection. Thus order automorphisms of any power set algebra $\mathcal{P}(X)$ are always given by the image of a permutation of $X$.

Example 4.5. Consider the two and three element sets $\{0,1\}$ and $\{0,1,2\}$. Then let $\sigma: \mathcal{P}(\{0,1\}) \rightarrow \mathcal{P}(\{0,1,2\})$ be defined by $\sigma(\emptyset)=\emptyset, \sigma(\{0\})=\{0\}, \sigma(\{1\})=\{1\}$ and $\sigma(\{0,1\})=\{0,1,2\}$. It is an order embedding that cannot be expressed as the image of any function $h$ on $\{0,1\}$.

4.2. Powers of $\mathbb{N}$. Now we examine order embeddings between powers of $\mathbb{N}$. These are the most important for purposes of this series of papers. Let $I$ be an index set. We have seen in example 2.28 that the product poset $\left(\mathbb{N}^{I}, \leq\right)$ is in fact a lattice monoid, and moreover is a complete semilattice satisfying a number of properties including infinite +-distributivity over $\vee$. Note that the lattice operations are given by $x \vee y=\max \{x, y\}$ and $x \wedge y=\min \{x, y\}$ where the max and min are taken coordinatewise, e.g. $\max \{f, g\}(i)=\max \{f(i), g(i)\}$ for all $i \in I$.

Notation 4.6. For $x \in \prod_{i \in I} X_{i}$ and $y \in \prod_{j \in J} Y_{j}$ we let $x \frown y$ denote the image of $(x, y)$ under the natural association between $\left(\prod_{i \in I} X_{i}\right) \times\left(\prod_{j \in J} Y_{i}\right)$ and $\prod_{i \in I \amalg J} Z_{i}$ where $Z_{i}=X_{i}$ for $i \in I$ and $Z_{j}=Y_{j}$ for $j \in J$. Similarly for functions, i.e. we write $f \frown g$ for $x \mapsto f(x) \frown g(x)$. 
Definition 4.7. Let $I$ be an index set. For a function $g: J \rightarrow I(J \subseteq I)$, the projection by $g$ is element $\pi_{g}: \mathbb{N}^{I} \rightarrow \mathbb{N}^{J}$ defined by

$$
\pi_{g}(x)=x \circ g .
$$

Proposition 4.8. $\pi_{g}$ is a continuous lattice homomorphism. It is an epimorphism iff $g$ is an injection, and it is a monomorphism iff $g$ is onto.

Notation 4.9. For $i \in I$, we let $\chi_{i} \in \mathbb{N}^{I}$ denote the characteristic function of $i$, i.e. $\chi_{i}(j)$ is 1 if $j=i$ and 0 if $j \neq i$. We write $\mathbf{0}_{I} \in \mathbb{N}^{I}$ for element that is constantly equal to 0 , i.e. $\mathbf{0}_{I}(i)=0$ for all $i \in I$. More generally, for $n \in \mathbb{N}$ write $\mathbf{n}_{I}$ for the element constantly equal to $n$.

Clearly, for every $x \in \mathbb{N}^{I}$,

$$
x=\bigvee_{i \in I} x(i) \cdot \chi_{i} .
$$

Theorem 4.10. The order embeddings $\sigma$ from $\left(\mathbb{N}^{I}, \leq\right)$ into $\left(\mathbb{N}^{J}, \leq\right)$ with convex range consist of maps of the form

$$
\sigma=\pi_{g}^{\frown} \mathbf{0}_{J \backslash \operatorname{dom}(g)}+y
$$

for some bijection $g: K \rightarrow I$ where $K \subseteq J$ and some $y \in \mathbb{N}^{J}$, and all such maps are dually continuous lattice embeddings. Thus $\sigma$ is the sum of a monoid homomorphism for coordinatewise addition and a constant.

Proof. We know that every order embedding of $\mathbb{N}^{I}$ into $\mathbb{N}^{J}$ with convex range is a dually continuous lattice homomorphism by corollary 3.43. Put $y=\sigma\left(\mathbf{0}_{I}\right)$. From corollary 3.29 and example 3.23 , we have that each $\chi_{i}$ is mapped to $k \cdot \chi_{j_{i}}+y$ for some $j_{i} \in J$. Applying proposition 3.26 with $\chi_{i} \in\left(\mathbb{N}^{I} \backslash\left\{\mathbf{0}_{I}\right\}\right)^{0}$, by convexity we see that $k=1$. Arguing similarly by induction, we obtain

$$
\sigma\left(k \cdot \chi_{i}\right)=k \cdot \chi_{j_{i}}+y \quad \text { for all } k \in \mathbb{N} .
$$

Thus $g: K \rightarrow I$ defined by $K=\left\{j_{i}: i \in I\right\}$ and

$$
g\left(j_{i}\right)=i
$$

is a bijection satisfying $\sigma\left(k \cdot \chi_{i}\right)=k \cdot \chi_{j_{i}}+y=\pi_{g}\left(k \cdot \chi_{i}\right) \mathbf{0}_{J \backslash K}+y$. Thus the continuity of $\sigma$, equation (4.3), proposition 4.8 and infinite $(+, \vee)$-distributivity yield

$$
\begin{aligned}
\sigma(x) & =\sigma\left(\bigvee_{i \in I} x(i) \cdot \chi_{i}\right)=\bigvee_{i \in I} \sigma\left(x(i) \cdot \chi_{i}\right)=\bigvee_{i \in I}\left(x(i) \cdot \chi_{j_{i}}+y\right) \\
& =\bigvee_{i \in I}\left(\pi_{g}\left(x(i) \cdot \chi_{i} \frown^{\frown} \mathbf{0}_{J \backslash K}+y\right)=\left(\bigvee_{i \in I} \pi_{g}\left(x(i) \cdot \chi_{i}\right)^{\frown} \mathbf{0}_{J \backslash K}\right)+y\right. \\
& =\pi_{g}(x) \frown \mathbf{0}_{J \backslash K}+y .
\end{aligned}
$$

Conversely, it is clear that whenever $g$ is a bijection and $y \in \mathbb{N}^{J}$ is arbitrary, $\sigma$ as above defines an embedding with convex range.

Corollary 4.11. Every map $\sigma: \mathbb{N}^{I} \rightarrow \mathbb{N}^{J}$ satisfying: 
(a) $x \leq y$ iff $\sigma(x) \leq \sigma(y)$, for all $x, y \in \mathbb{N}^{I}$,

(b) $\sigma(x) \leq z \leq \sigma(y)$ implies $z \in \operatorname{ran}(\sigma)$, for all $x, y \in \mathbb{N}^{I}$ and $z \in \mathbb{N}^{J}$,

is of the form

$$
\sigma=\pi_{g}^{\frown} \mathbf{0}_{J \backslash \operatorname{dom}(g)}+\sigma\left(\mathbf{0}_{I}\right)
$$

for some bijection $g: K \rightarrow I$ where $K \subseteq J$.

Proof. Theorem 4.10 applies as conditions (a) and (b) translate to $\sigma$ being an embedding with convex range.

Corollary 4.12. Any embedding $\sigma: \mathbb{N}^{I} \rightarrow \mathbb{N}^{J}$ with convex range has $\operatorname{ran}(\sigma)=$ $\mathbb{N}^{K} \mathbf{0}_{J \backslash K}+y$ for some $K \subseteq J$ and $y \in \mathbb{N}^{J}$

Proof. By theorem 4.10 because $\operatorname{ran}\left(\pi_{g}\right)=\mathbb{N}^{K}$ since $g$ is injective.

Corollary 4.13. Every order embedding $\sigma$ of $\mathbb{N}^{I}$ into a downwards closed subset of $\mathbb{N}^{J}$ is of the form

$$
\sigma=\pi_{g}^{\frown} \mathbf{0}_{J \backslash \operatorname{dom}(g)}
$$

for some bijection $g: K \rightarrow I$ where $K \subseteq J$, and thus has $\operatorname{ran}(\sigma)=\mathbb{N}^{K} \frown_{J \backslash K}$. In particular, it is a monoid homomorphism.

Corollary 4.14. Every quasi order isomorphism $\sigma: \mathbb{N}^{I} \rightarrow \mathbb{N}^{J}$ is of the form $\pi_{g}$ where $g: J \rightarrow I$ is a bijection. Thus order automorphisms of $\mathbb{N}^{I}$ are all of the form $\pi_{g}$ where $g$ is a permutation of $I$.

4.3. Category algebras. To start we review the basic concepts. Let $X$ be a topological space. We let $\mathcal{M}(X)$ denote the $\sigma$-ideal of meager subsets of $X$ (i.e. countable unions of nowhere dense sets). Recall that $X$ is a Baire space if it has no nonempty open meager sets. And recall that $B \subseteq X$ has the Baire property if it can be approximated by an open set; that is there is an open $U$ such that $B \triangle U \in \mathcal{M}(X)$. We write $\operatorname{BP}(X)$ for the $\sigma$-algebra of subsets of $X$ with the Baire property. The category algebra of $X$ is the quotient algebra $\mathrm{BP}(X) / \mathcal{M}(X)$, i.e. equivalence classes of $\mathrm{BP}(X)$ modulo $A \triangle B \in \mathcal{M}(X)$ ordered by $[A] \leq[B]$ if $A \backslash B \in \mathcal{M}(X)$. It is a complete Boolean algebra, with $[A] \vee[B]=[A \cup B],[A] \wedge[B]=[A \cap B]$ and $-[A]=\left[A^{\complement}\right]$. We shall denote the category algebra by $\operatorname{Cat}(X)=\operatorname{BP}(X) / \mathcal{M}(X)$. Recall that a subset $G \subseteq X$ is regular open if it is equal to the interior of its closure; symbolically, $G=\bar{G}^{\circ}$, i.e. $\bar{G}$ denotes the topological closure of $G$ while $H^{\circ}$ denotes the interior of $H$. We write $\operatorname{RO}(X)$ for the regular open algebra of $X$. It is a complete Boolean with $G \vee H=\overline{G \cup H}^{\circ}, G \wedge H=G \cap H$ and $-G=\bar{G}^{\complement}$. A topological space is semiregular if its regular open sets form a base for its topology.

We shall make use of the following topological facts.

Theorem 4.15 (Baire Category Theorem). Every locally compact Hausdorff space is a Baire space.

Proposition 4.16. If $\mathcal{U}$ is a collection of pairwise disjoint open sets, and $M_{U} \subseteq U$ is meager for all $U \in \mathcal{U}$, then $\bigcup_{U \in \mathcal{U}} M_{U}$ is meager.

Proof. The main observations are that for any open set $U, \bar{U} \backslash U$ is nowhere dense, and that the collection of nowhere dense sets forms an ideal. 
Proposition 4.17. Let $X$ be a topological space. Then it has a largest open meager set $U_{X}$. And $G \mapsto[G]$ is a Boolean algebra isomorphism between $\operatorname{RO}\left(X \backslash \bar{U}_{X}\right)$ and $\operatorname{Cat}(X)$. Thus if $X$ is a Baire space then its category algebra is isomorphic to its regular open algebra.

Proof. Use proposition 4.16.

Proposition 4.18. If $X$ is regular then it is semiregular, and in particular every nonempty open subset of $X$ contains a nonempty regular open subset.

Definition 4.19. We shall call $Y$ a Baire compactification of a topological space $X$, if

(i) $Y$ is a compact Hausdorff space,

(ii) $X$ topologically embeds into $Y$, i.e. there is a map $f: X \rightarrow Y$ such that $f$ is a homeomorphism between $X$ and $f[X]$,

(iii) $f[X]$ is comeager in $Y$.

Thus condition (iii) is a strengthening of the usual notion of a compactification where it is only required that $f[X]$ is dense.

Example 4.20. Locally compact Hausdorff spaces have a Baire compactification since they in fact have a one point compactification.

Proposition 4.21. Any topological space with a Baire compactification is a Baire space.

Proof. Straight from definition 4.19,

Lemma 4.22. Every Polish space (i.e. separable completely metrizable space) has a metric Baire compactification.

Proof. See e.g. [Kec95].

Example 4.23. The irrationals $\mathbb{N}^{\mathbb{N}}$ with the product topology, which are homeomorphic to the irrationals of the real line, is a Polish space.

Proposition 4.24. If $Y$ is a Baire compactification of $X$ then $\operatorname{Cat}(Y)$ is isomorphic as a Boolean algebra to $\operatorname{Cat}(X)$.

Proof. Identifying $X$ as a comeager subset of $Y,[B] \mapsto[B \cap X]$ is an isomorphism between $\mathrm{BP}(Y) / \mathcal{M}(Y)$ and $\mathrm{BP}(X) / \mathcal{M}(X)$.

Theorem 4.25. Let $X$ be a regular space, and suppose $Y$ has a Baire compactification. The order embeddings $\sigma: \operatorname{Cat}(X) \rightarrow \operatorname{Cat}(Y)$ with convex range consist of all maps of the form

$$
\sigma([B])=[f[B]] \vee[A] \quad \text { for all } B \in \mathrm{BP}(X),
$$

for some Baire measurable $f: X \rightarrow Y$ and $A \in \mathrm{BP}(Y)$ such that

(a) $f[B] \in \mathrm{BP}(Y)$ for all $B \in \mathrm{BP}(X)$,

(b) $M \in \mathcal{M}(X)$ iff $f[M] \in \mathcal{M}(Y)$ for all $M \subseteq X$,

(c) $f[B] \cap f\left[B^{\complement}\right] \in \mathcal{M}(Y)$ for all $B \in \operatorname{BP}(X)$,

(d) $f[X] \cap A \in \mathcal{M}(Y)$. 
Moreover, any such map is a dually continuous lattice embedding. Furthermore, $f[X]$ is comeager iff $\sigma$ is a Boolean algebra isomorphism.

Proof. We can simplify by assuming that $X$ is a Baire space by proposition 4.17 since clearly $\mathrm{BP}\left(X \backslash \bar{U}_{X}\right) / \mathcal{M}\left(X \backslash \bar{U}_{X}\right) \cong \mathrm{BP}(X) / \mathcal{M}(X)$. Let $\bar{\sigma}: \operatorname{Cat}(X) \rightarrow \operatorname{Cat}(Y)$ be an order embedding with convex range. Then $\bar{\sigma}^{\prime}: \operatorname{Cat}(X) \rightarrow \operatorname{Cat}(Y)$ given by $\bar{\sigma}^{\prime}(a)=\bar{\sigma}(a)-\bar{\sigma}\left(0_{\operatorname{Cat}(X)}\right)$ has a downwards closed range, and it will suffice to prove that it is of the form

$$
\bar{\sigma}^{\prime}([B])=[f[B]] \quad \text { for all } B \in \mathrm{BP}(X)
$$

for a Baire function $f: X \rightarrow Y$ satisfying (国)-(远), because then taking $[A]=$ $\bar{\sigma}\left(0_{\mathrm{Cat}(X)}\right)$, condition (d) follows from (4.9) and (a) and (Bb) since $\bar{\sigma}$ is $1-1$. By going to a Baire compactification via proposition 4.24, we can assume that $Y$ is compact and Hausdorff. Note that $Y$ is a Baire space by the Baire Category Theorem (theorem 4.15). Hence, by proposition 4.17, we can define $\sigma: \operatorname{RO}(X) \rightarrow \operatorname{RO}(Y)$ by $[\sigma(G)]=\bar{\sigma}^{\prime}([G])$ and thus obtain an embedding with downwards closed range.

For each $z \in X$, let

$$
\mathcal{G}_{z}=\{G \in \operatorname{RO}(X): G \ni z\},
$$

and

$$
K_{z}=\bigcap_{G \in \mathcal{G}_{z}} \overline{\sigma(G)} .
$$

Since $\mathcal{G}_{z}$ is closed under finite intersections, $K_{z} \neq \emptyset$ by compactness. Now choose $f: X \rightarrow Y$ so that $f(z) \in K_{z}$ for all $z \in X$.

Claim 4.26. $G \triangle f^{-1}[\sigma(G)] \in \mathcal{M}(X)$ for all $G \in \operatorname{RO}(X)$.

Proof. Let $\mathcal{V}=\{H \in \operatorname{RO}(X): \overline{\sigma(H)} \subseteq \sigma(G)\}$. Since $z \in H$ and $\overline{\sigma(H)} \subseteq G$ imply $f(z) \in K_{z} \subseteq \overline{\sigma(H)} \subseteq \sigma(G)$, and since $\sigma(H) \subseteq \sigma(G)$ implies $H \subseteq G$ by order reflection,

$$
\bigcup \mathcal{V} \subseteq G \cap f^{-1}[\sigma(G)]
$$

On the other hand, letting $\mathcal{W}=\left\{H \in \operatorname{RO}(X): \sigma(H) \subseteq \sigma(G)^{\complement}\right\}$, since $z \in H$ and $\overline{\sigma(H)} \subseteq \sigma(G)^{\complement}$ imply $f(z) \notin \sigma(G)$, and since $\sigma(H) \subseteq \sigma(G)^{\complement}$ implies $H \cap G=\emptyset$ by order preservation, we obtain

$$
\bigcup \mathcal{W} \subseteq\left(G \cup f^{-1}[\sigma(G)]\right)^{\complement} .
$$

Therefore, as $\left(G \triangle f^{-1}[\sigma(G)]\right) \cap(\bigcup \mathcal{V} \cup \cup \mathcal{W})=\emptyset$, it remains to show that $\bigcup \mathcal{V} \cup \bigcup \mathcal{W}$ is comeager. But if this were not the case, then there would exist $H \in \operatorname{RO}(X)^{+}$ such that

$$
H \cap \bigcup \mathcal{V} \cup \bigcup \mathcal{W}=\emptyset
$$

And in particular $H \notin \mathcal{W}$, and thus $\sigma(H) \cap \sigma(G) \neq \emptyset$. Then since $Y$ is in particular regular, there exists a nonempty $Z \in \mathrm{RO}(Y)$ with $\bar{Z} \subseteq \sigma(H) \cap \sigma(G)$ by proposition 4.18. Now since the range of $\sigma$ is downwards closed, there exists a nonempty 
$E \in \mathrm{RO}(X)^{+}$such that $\sigma(E)=Z$, and hence $E \in \mathcal{V}$. However, by order reflection $E \subseteq H$, contradicting (4.14).

Claim 4.27. $f^{-1}[H] \in \mathrm{BP}$ for all $H \in \mathrm{RO}(Y)$.

Proof. We have $f^{-1}[H]=f^{-1}[H \cap \sigma(X)] \cup f^{-1}[H \backslash \sigma(X)]$, and thus $f^{-1}[H] \triangle f^{-1}[H \cap$ $\sigma(X)] \in \mathcal{M}$, since $f^{-1}[\sigma(X)]$ is comeager by claim 4.26. It thus suffices to prove $f^{-1}[H \cap \sigma(X)] \in \mathrm{BP}$. But by downwards closedness, there exists $G \in \operatorname{RO}(X)$ such that $\sigma(G)=H \cap \sigma(X)$. Thus the result follows by applying claim 4.26 to $G$.

Claim 4.28. $f^{-1}[[\mathcal{M}]] \subseteq \mathcal{M}$, i.e. $f^{-1}[M] \in \mathcal{M}(X)$ for all $M \in \mathcal{M}(Y)$.

Proof. It suffices to prove that $f^{-1}[F]$ is a nowhere dense set for every nowhere dense $F \subseteq Y$. Let $U \subseteq X$ be a given nonempty set. We need to find a nonempty open $V \subseteq U$ such that $f[V] \cap F=\emptyset$. By proposition 4.18 , there exists $G \subseteq U$ in $\operatorname{RO}(X)^{+}$. Then there exists $E \leq \sigma(G)$ in $\operatorname{RO}(Y)^{+}$such that $\bar{E} \cap F=\emptyset$. And there exists $H \in \mathrm{RO}(X)^{+}$such that $\sigma(H)=E$. Moreover, by order reflection, $H \subseteq G \subseteq U$. Now $f[H] \subseteq \overline{\sigma(H)}$ and thus $f[H] \cap F=\emptyset$, as needed.

Claim 4.29. $f[[\mathcal{M}]] \subseteq \mathcal{M}$.

Proof. Let $F \subseteq X$ be nowhere dense. It suffices to prove that for all $H \in \operatorname{RO}(Y)^{+}$, there exists $Z \subseteq H$ in $\operatorname{RO}(Y)^{+}$such that $Z \cap f[F]=\emptyset$. Given said $H$, if $H \cap \sigma(X)=\emptyset$ then since $f[X] \subseteq \overline{\sigma(X)}$, we already have $H \cap f[F]=\emptyset$. Otherwise, there exists $G \in \operatorname{RO}(X)^{+}$such that $\sigma(G) \subseteq H$. Since $G \nsubseteq \bar{F}$, by regularity there exist $Z, W \in$ $\mathrm{RO}(X)^{+}$such that $Z \subseteq G, W \supseteq F$ and $W \cap Z=\emptyset$. Clearly $f[F] \subseteq \overline{\sigma(W)}$, and since $\sigma(W) \cap \sigma(Z)=\emptyset$ which implies $\overline{\sigma(W)} \cap \sigma(Z)=\emptyset, f[F] \cap \sigma(Z)=\emptyset$. By order preservation, $\sigma(Z) \subseteq H$, completing the proof.

Claim 4.30. $f[G] \triangle \sigma(G) \in \mathcal{M}(Y)$ for all $G \in \mathrm{RO}(X)$.

Proof. First we note that $\sigma(G) \backslash f[G] \in \mathcal{M}$. For supposing to the contrary, there exists $H \subseteq \sigma(G)$ in $\operatorname{RO}(Y)^{+}$such that

$$
H \cap f[G] \in \mathcal{M} .
$$

By the assumption on $\operatorname{ran}(\sigma)$, there is an $E$ such that $\sigma(E)=H$, and $E \subseteq G$ by order reflection. Now $E=\left(E \cap f^{-1}[H]\right) \cup\left(E \backslash f^{-1}[H]\right)$, and thus $E \cap f^{-1}[H] \notin \mathcal{M}$ by claim 4.26. But $E \cap f^{-1}[H] \subseteq f^{-1}[H \cap f[G]] \in \mathcal{M}$ by (4.15) and claim 4.28, a contradiction.

On the other hand $f[G] \subseteq \overline{\sigma(G)}$ proving $f[G] \backslash \sigma(G) \in \mathcal{M}$.

By claims 4.27 and 4.28, $f$ is Baire measurable. And by claim 4.30, $\bar{\sigma}^{\prime}([G])=$ $[\sigma(G)]=[f[G]]$ for all $G \in \operatorname{RO}(X)$. Note that condition (b) is equivalent to the conjunction of claims 4.28 and 4.29, And now (国) follows, and moreover the expression in (4.9) is well defined, i.e. $[f[B]]$ does not depend on the choice of $B \in[G]$. As for condition ( $(\mathbb{C})$, given $B \in \operatorname{BP}(X)$ it suffices to take the $G \in \operatorname{RO}(X)$ with $[G]=[B]$, and observe that $f\left[G^{\mathrm{C}}\right] \triangle f\left[\bar{G}^{\mathrm{C}}\right] \in \mathcal{M}(Y)$ by (b) , and thus $\left[f[G] \cap f\left[G^{\mathrm{C}}\right]\right]=$ $[f[G]] \wedge\left[f\left[\bar{G}^{\mathrm{C}}\right]\right]=[\sigma(G)] \wedge\left[\sigma\left(\bar{G}^{\mathrm{C}}\right)\right]=[\sigma(G)] \wedge[-\sigma(G)]=0_{\mathrm{Cat}(Y)}$.

Conversely, suppose that $f: X \rightarrow Y$ and $A \in \mathrm{BP}(Y)$ satisfy the hypotheses. Then $\sigma$ determined by (4.8) makes sense and is well defined by (a) and (b), and convexity of 
the range follows from the Baire measurability of $f$. It is order preserving because $[B] \leq[C]$ implies that $f[B] \backslash f[C] \subseteq f[B \backslash C] \in \mathcal{M}$ by (b), which implies that $\sigma([B])-\sigma([C])=0_{\operatorname{Cat}(Y)}$. And it is order reflecting because $\sigma([B])-\sigma([C])=0_{\operatorname{Cat}(Y)}$ implies $f[B] \backslash f[C] \in \mathcal{M}$ by (dd), and this implies that $f[B \backslash C] \in \mathcal{M}$ : For

$$
f[B] \cap f[C]^{\complement}=f[B] \backslash f[C] \subseteq f[B \backslash C] \subseteq f[B] \cap f\left[C^{\complement}\right]
$$

and $\left(f[B] \cap f\left[C^{\complement}\right]\right) \backslash\left(f[B] \cap f[C]^{\complement}\right) \subseteq f[C] \cap f\left[C^{\complement}\right] \in \mathcal{M}$ by (드). Therefore, $B \backslash C \in \mathcal{M}$ by (b) as required.

In the context of Boolean algebra homomorphisms, it would be more natural to use $\bar{f}^{-1}$ for some $\bar{f}: Y \rightarrow X$ rather than $f: X \rightarrow Y$ since this would allow for homomorphisms that are not monomorphisms. We reformulate the preceding theorem in this manner.

Corollary 4.31. Let $X$ be a regular space, and let $Y$ have a Baire compactification. Then order embeddings of $\operatorname{Cat}(X)$ into $\operatorname{Cat}(Y)$ with convex range consist precisely of maps of the form

$$
\sigma([B])=\left[f^{-1}[B]\right] \vee[A] \text { for all } B \in \mathrm{BP}(X),
$$

for some $Z \in \mathrm{BP}(Y)$ and some Baire measurable injection $f: Z \rightarrow X$ such that

(a) $f[C] \in \mathrm{BP}(X)$ for all $C \in \mathrm{BP}(Z)$,

(b) $M \in \mathcal{M}(X)$ iff $f^{-1}[M] \in \mathcal{M}(Y)$ for all $M \subseteq X$,

(c) $Z \cap A \in \mathcal{M}(Y)$.

Furthermore, $Z$ is comeager iff $\sigma$ is a Boolean algebra isomorphism.

Proof. Supposing $\sigma$ is an order embedding, let $\bar{f} \in \operatorname{Baire}(X, Y)$ and $A \in \operatorname{BP}(Y)$ satisfy the conclusion of theorem 4.25. Put $Z=\operatorname{ran}(\bar{f})$. Choose a right inverse $f: Z \rightarrow X$ of $\bar{f}$. The fact that it is a right inverse entails

$$
f^{-1}[B] \subseteq \bar{f}[B] \text { for all } B \subseteq X .
$$

And plugging in $B^{\complement}$ for $B$ yields $\bar{f}\left[B^{\complement}\right]^{\complement} \subseteq f^{-1}[B]$ for all $B \subseteq X$. Therefore

$$
\bar{f}[B] \backslash f^{-1}[B] \subseteq \bar{f}[B] \cap \bar{f}\left[B^{\complement}\right] \in \mathcal{M}(Y) \text { for all } B \in \operatorname{BP}(X)
$$

by condition ( $(\mathbb{C})$ for $\bar{f}$. It now follows from the conclusion of theorem 4.25 that $\sigma$ satisfies equation (4.17) and that $f$ is a Baire measurable function satisfying (b) and (드). Condition (国) can be verified similarly.

The converse is completely straightforward.

It seems likely to us that the preceding corollary is known for the special case of Boolean algebra isomorphisms (i.e. $Z$ comeager), although we do not know of it in the literature, except in the specific case of some explicit Polish spaces $X$ and $Y$.

Corollary 4.32. Let $X$ be regular and $Y$ have a Baire compactification. The range of any order embedding $\sigma: \operatorname{Cat}(X) \rightarrow \operatorname{Cat}(Y)$ with convex range is the interval Boolean algebra $\left[\sigma\left(0_{\operatorname{Cat}(X)}\right), \sigma\left(1_{\operatorname{Cat}(X)}\right)\right]$, and $i^{-1} \circ \sigma$ is a Boolean algebra isomorphism between $\operatorname{Cat}(X)$ and the interval Boolean algebra, where $i$ is the inclusion map.

In the second countable case we get a sharper description of $f$. 
Corollary 4.33. Let $X$ be a second countable Hausdorff space, and let $Y$ be a Polish space. The order embeddings $\sigma: \operatorname{Cat}(X) \rightarrow \operatorname{Cat}(Y)$ with convex range consist of all of the maps of the form

$$
\sigma([B])=[f[B \cap C]] \vee[A] \text { for all } B \in \mathrm{BP}(X),
$$

for some comeager $C \subseteq X, f: C \rightarrow Y$ and $A \subseteq Y$ such that

(a) $f$ is a topological embedding,

(b) $f[C] \in \mathrm{BP}(Y)$,

(c) $f[C] \cap A \in \mathcal{M}(Y)$.

Proof. Let $\sigma$ be an embedding with convex range. Note that $X$ is regular, because $X$ is metrizable (by Urysohn's Metrization Theorem), and note that $Y$ has a Baire compactification by lemma 4.22. By theorem 4.25, there is a Baire measurable $f: X \rightarrow Y$ and $A \in \mathrm{BP}(Y)$ such that

$$
\sigma([B])=[f[B]] \vee[A] \text { for all } B \in \mathrm{BP}(X),
$$

$M \in \mathcal{M}(X)$ iff $f[M] \in \mathcal{M}(Y)$ for all $M \subseteq X$,

$$
f[B] \cap f\left[B^{\complement}\right] \in \mathcal{M}(Y) \text { for all } B \in \operatorname{BP}(X),
$$

Since $Y$ is a Baire space by proposition 4.21 , we can define $\bar{\sigma}: \mathrm{RO}(X) \rightarrow \mathrm{RO}(Y)$ by letting $\bar{\sigma}(G)$ be the unique member of $\mathrm{RO}(Y)$ such that $[\bar{\sigma}(G)]=\sigma([G])-[A]$. Note that $\bar{\sigma}$ is an embedding with downwards closed range because $[A]=\sigma\left(0_{\mathcal{M}(X)}\right)$ by (4.21).

Then equations (4.21) and (4.24) together imply that $f[G] \triangle \bar{\sigma}(G) \in \mathcal{M}(Y)$ for all $G \in \mathrm{RO}(X)$, and equation (4.23) implies that $f[G] \cap f\left[G^{\mathrm{C}}\right] \in \mathcal{M}(Y)$ for all $G \in \operatorname{RO}(X)$. As $f^{-1}[f[G] \triangle \bar{\sigma}(G)] \in \mathcal{M}(X)$ and $f^{-1}\left[f[G] \cap f\left[G^{\mathrm{C}}\right]\right] \in \mathcal{M}(X)$ for all $G$ by (4.22), and as $X$ is second countable, we can find a comeager $C \subseteq X$ such that

$$
\begin{array}{r}
f[C] \cap(f[G] \triangle \bar{\sigma}(G))=\emptyset, \\
f[C] \cap f[G] \cap f\left[G^{\complement}\right]=\emptyset,
\end{array}
$$

for all $G \in \operatorname{RO}(X)$. Therefore,

$$
f[G \cap C]=\bar{\sigma}(G) \cap f[C] \quad \text { for all } G \in \operatorname{RO}(X),
$$

because $f[G \cap C]=f[G] \cap f[C]$ by (4.26), and $f[G] \cap f[C]=\bar{\sigma}(G) \cap f[C]$ by (4.25). By the semiregularity of $X$ this implies that $f \uparrow C$ maps all opens sets to relative open subsets of its range. And since $X$ is Hausdorff, every two distinct points in $C$ have disjoint regular open neighbourhoods, and thus their images under $f \uparrow C$ are disjoint by (4.27) since $\bar{\sigma}$ is an embedding; hence, $f\lceil C$ is $1-1$. And by shrinking $C$, since $Y$ is in particular second countable, we can moreover arrange that $f \uparrow C$ is continuous (by Baire's Theorem). Therefore $f \uparrow C$ is a topological embedding, while (b) and ( (C) are consequences of (4.21) and (4.24), respectively.

Conversely, it is clear that any $C \subseteq X, f: C \rightarrow Y$ and $A$ satisfying the hypotheses define an order embedding $\sigma$ with convex range via (4.20). 
4.4. Baire functions into powers of $\mathbb{N}$ modulo almost always equality. The results in this section will play a crucial role in the next paper Hir06a of this series. The main results are theorem 4.40 where a number of properties of the monoid Baire $\left(X, \mathbb{N}^{I}\right) /={ }_{\text {aa }}$ are established; theorem 4.50 where embeddings of $\operatorname{Baire}\left(X, \mathbb{N}^{I}\right) /={ }_{\text {aa }}$ into $\operatorname{Baire}\left(Y, \mathbb{N}^{J}\right) /=_{\text {aa }}$ with convex range are characterized for arbitrary topological spaces $X$ and $Y$; and theorem 4.62 where these embeddings, for $X$ regular and $Y$ having a Baire compactification, are described in terms of generalized projections (definition 4.61).

Before preceding, we recall that a Baire measurable function is of course a function $f: X \rightarrow Y$ between two topological spaces such that $f^{-1}(U) \in \operatorname{BP}(X)$ for every open $U \subseteq Y$. We often just call them Baire functions (not to be confused with the different concept of Baire class $n$ functions) and we write Baire $(X, Y)$ for the family of Baire functions from $X$ into $Y$. And we write $\operatorname{Cat}(X, Y)$ for the family of continuous functions $X$ into $Y$.

Given some relation $R$ on a topological space $S$, and given a topological space $X$, we would like to use $R$ to induce a relation on $\operatorname{Baire}(X, S)$ in the sense of "almost always" in the space $X$. However, we shall see that this is not as straightforward as it may seem.

It will be constructive to express a given relation as a conjunction. Suppose then that $\mathcal{R}$ is a family of binary relations on a fixed space $S$. We define a relation $\mathcal{R}_{\text {aa }}$ on $\operatorname{Baire}(X, S)$ by

$$
f \mathcal{R}_{\text {aa }} g \text { if } \bigwedge_{R \in \mathcal{R}}\ulcorner f(z) R g(z) \text { for almost all } z \in X\urcorner,
$$

where "for almost all" is interpreted as comeagerly many. Thus we are generalizing the relation $\bigwedge \mathcal{R}$ from $S$ to $\operatorname{Baire}(X, S)$. However, the resulting relation on the function space may depend on the representation when the family $\mathcal{R}$ is uncountable, as shown in example 4.38 below. Also note that the definition in (4.28) can be generalized from binary relations to arbitrary $n$-place relations.

Proposition 4.34. If each $R \in \mathcal{R}$ is a quasi ordering of $S$, then $\mathcal{R}_{\text {aa }}$ is a quasi ordering of $\operatorname{Baire}(X, S)$.

Example 4.35. We consider the relation $\leq$ on $\mathbb{N}^{I}$. For each $i \in I$, let $R_{i}$ be the relation satisfying $x R_{i} y$ iff $x(i) \leq y(i)$. Then $\leq=\bigwedge_{i \in I} R_{i}$. Putting $\mathcal{R}=\left\{R_{i}: i \in\right.$ $I\}$ we obtain the following relation on $\operatorname{Baire}\left(X, \mathbb{N}^{I}\right)$ :

$$
f \mathcal{R}_{\text {aa }} g \quad \text { iff } \quad f(z)(i) \leq g(z)(i) \text { for almost all } z \in X \quad \text { for all } i \in I .
$$

Let us denote $\mathcal{R}_{\text {aa }}$ from equation (4.29) as $\leq_{\text {aa }}$. We repeat this construction for the equality relation. Thus we let $\mathcal{S}=\left\{S_{i}: i \in I\right\}$ where $x S_{i} y$ iff $x(i)=y(i)$, and let $={ }_{\text {aa }}$ denote the relation $\mathcal{S}_{\mathrm{aa}}$. These are both quasi orderings of the family of Baire functions by proposition 4.34

These are the 'correct' representations of $\leq$ and $=$, because of the following connection with set theoretic forcing. We shall not attempt to explain the forcing concepts here (see e.g. [Kun80]). Noting that $=_{a a}$ is an equivalence relation on 
Baire $\left(X, \mathbb{N}^{I}\right)$, we observe that there are Borel representatives of the Baire measurable functions.

Lemma 4.36. Let $X$ be a topological space. Then Baire $\left(\mathbb{N}^{I}\right) /={ }_{\mathrm{aa}}$ is equal to $\operatorname{Borel}\left(\mathbb{N}^{I}\right) /={ }_{\text {aa }}$.

Proof. By our representation $\mathcal{S}$ of equality, it suffices to observe that every Baire function $f: X \rightarrow \mathbb{N}$ has a Borel function $g_{f}: X \rightarrow \mathbb{N}$ with $[f]={ }_{\text {aa }}\left[g_{f}\right]$. Then given $[F] \in \operatorname{Baire}\left(\mathbb{N}^{I}\right) /=$ aa we can define $G: X \rightarrow \mathbb{N}^{I}$ by $G(z)(i)=g_{F(\cdot)(i)}(z)$ for all $z \in X$ and $i \in I$, which yields a Borel function $G$ with $[G]={ }_{\text {aa }}[F]$.

Note too that:

Proposition 4.37. If $f \in \operatorname{Baire}\left(X, \mathbb{N}^{I}\right)$ and $g={ }_{\text {aa }} f$ then $g \in \operatorname{Baire}\left(X, \mathbb{N}^{I}\right)$.

Given $f \in \operatorname{Baire}\left(X, \mathbb{N}^{I}\right)$, we let $f^{\prime} \in \operatorname{Borel}\left(X, \mathbb{N}^{I}\right)$ satisfy $\left[f^{\prime}\right]={ }_{\text {aa }}[f]$. The advantage of Borel sets and Borel functions is that they have an explicit description that allows them to be interpreted in new larger "universes" extending the present one (e.g. forcing extensions), that may contain e.g. new real numbers. Now we make three assumptions on the topological space $X$. Firstly, we assume that it is given by an explicit definition, by which we of course mean a formula of set theory, possibly with a parameter. This is true of any set $X$, but the point is that we might consider a formula $\varphi(x)$ defining the real line $\mathbb{R}$ for example, so that when we go to a forcing extension the same formula gives new reals. Secondly, we assume the defined space $X$ is Hausdorff, i.e. in any model extending our present universe and satisfying enough of the axioms of set theory, the space $X$ defined by the formula $\varphi(x)$ is Hausdorff. Then for any sufficiently generic filter $G$ on the poset $(\operatorname{Borel}(X) \backslash \mathcal{M}(X), \subseteq)$, which produces the same forcing extension as $\operatorname{Cat}(X), \bigcap G$ has at most one element when the intersection is interpreted in the forcing extension by $\operatorname{Cat}(X)$. Thirdly, we require that the defined space satisfies some topological 'completeness' so that $\bigcap G \neq \emptyset$; this is where we want to use a definition of a topological space rather than a fixed space, because a fixed space may lose its completeness property in a larger universe. We will not explicitly formulate this notion of completeness, but we note that complete metrizability is sufficient although there is no need to restrict ourselves to metric spaces. Under these three assumptions, we let $\dot{g}$ denote the single element of $\bigcap G$ in the forcing extension by $\operatorname{Cat}(X)$. Back to the function $f$, we write $\dot{f}$ for $f^{\prime}(\dot{g})$ where the Borel function $f^{\prime}$ is being interpreted in the forcing extension by $\operatorname{Cat}(X)$.

Now the "forcing theorem" applied to the forcing notion Cat $(X)$ and elements of $\mathbb{N}^{I}$ states that for every formula $\varphi\left(x_{0}, \ldots, x_{n-1}\right)$ of the language of set theory, for all $f_{0}, \ldots, f_{n-1} \in \operatorname{Baire}\left(X, \mathbb{N}^{I}\right)$,

$$
\operatorname{Cat}(X) \Vdash \varphi\left(\dot{f}_{0}, \ldots, \dot{f}_{n-1}\right)
$$


In particular, for all $f, g \in \operatorname{Baire}\left(X, \mathbb{N}^{I}\right)$,

$$
\begin{array}{lll}
\operatorname{Cat}(X) \Vdash \dot{f}=\dot{g} & \text { iff } & f={ }_{\text {aа }} g, \\
\operatorname{Cat}(X) \Vdash \dot{f} \leq \dot{g} & \text { iff } & f \leq_{\text {aa }} g .
\end{array}
$$

In set theoretic terminology, every member of $\operatorname{Baire}\left(X, \mathbb{N}^{I}\right)$ 'names' a member of $\mathbb{N}^{I}$ lying in the forcing extension by $\operatorname{Cat}(X)$, and visa versa. Thus equations (4.31) and (4.32) state that $f$ and $g$ name the same object iff $f={ }_{\text {aa }} g$, and $f$ names a member of $\mathbb{N}^{I}$ below the member named by $g$ iff $f \leq_{\text {aa }} g$. This correspondence has been known for a long time; for example, the measure theoretic analogue for measure algebras (discussed in \$4.6.2) appears in 1967 ([Sco67]). Indeed Cat $(X)$ is (isomorphic as a forcing notion to) the original forcing notion used by Cohen (Coh63, Coh64]) to prove that the Continuum Hypothesis is independent of the usual axioms of mathematics.

On the other hand, we consider the 'obvious' representation of equality to be 'incorrect'. Considering the singleton $\{=\}$, we obtain following generalization of equality to the space $\operatorname{Baire}\left(X, \mathbb{N}^{I}\right)$ of Baire functions:

$$
f\{=\}_{\text {aa }} g \quad \text { iff } \quad f(z)=g(z) \text { for almost all } z \in X .
$$

If the index set $I$ is countable then $\{=\}_{\text {aa }}$ is equivalent to $=_{\text {aa }}$ because $\mathcal{M}(X)$ is a $\sigma$-ideal. However, if the index set is of larger cardinality then these relations may differ, as in the following example 4.38. Also lemma 4.36 may fail with $\{=\}_{\text {aa }}$.

Example 4.38. Define $f \in \operatorname{Baire}\left(\mathbb{R}, \mathbb{N}^{\mathbb{R}}\right)$ by

$$
f(z)(x)= \begin{cases}1, & \text { if } x=z, \\ 0, & \text { if } x \neq z .\end{cases}
$$

Then $f={ }_{\text {aa }} \mathbf{0}_{\mathbb{R}}$; however, it is not the case that $f\{=\}_{\text {aa }} \mathbf{0}_{\mathbb{R}}$, because $f(z) \neq \mathbf{0}_{\mathbb{R}}$ for all $z \in \mathbb{R}$.

Perhaps the most basic aspect of $=_{a \mathrm{a}}$ is that it gives a product order.

Proposition 4.39. $[f] \mapsto([f(\cdot)(i)]: i \in I)$ defines an isomorphism between $\operatorname{Baire}\left(X, \mathbb{N}^{I}\right) /=_{\text {aa }}$ and $\left(\operatorname{Baire}(X, \mathbb{N}) /={ }_{\text {aa }}\right)^{I}$.

Observe that for all $f, g \in \operatorname{Baire}\left(X, \mathbb{N}^{I}\right), f \leq_{\text {aa }} g$ and $g \leq_{\text {aa }} f$ iff $f={ }_{\text {aa }} g$, and thus the poset (Baire $\left.\left(X, \mathbb{N}^{I}\right) /=_{\mathrm{aa}}, \leq_{\mathrm{aa}}\right)$ is the antisymmetric quotient of (Baire $\left(X, \mathbb{N}^{I}\right)$, $\leq_{\text {aa }}$ ) (cf. (3). Also (Baire $\left(X, \mathbb{N}^{I}\right), \leq$ ), where $f \leq g$ means $f(z) \leq g(z)$ for all $z \in X$, is clearly a lattice where $f \vee g=\max \{f, g\}$ and $f \wedge g=\min \{f, g\}$ are taken pointwise:

$$
\max \{f, g\}(z)=\max \{f(z), g(z)\} \quad \text { and } \min \{f, g\}(z)=\min \{f(z), g(z)\}
$$

for all $z \in X$ (cf. \$4.2). Furthermore, (Baire $\left(X, \mathbb{N}^{I}\right),+$ ), where addition is taken pointwise, is clearly a commutative monoid (cf. example 2.28). In fact, it is a lattice monoid where the monoid ordering $\leq$ is the above lattice order. And it is easily verified that $=_{\mathrm{aa}}$ is a congruence for this monoid (cf. \$1.1), and thus the quotient $\operatorname{Baire}\left(X, \mathbb{N}^{I}\right) /=_{\text {aa }}$ is a quotient monoid with the monoid operation

$$
[f]+[g]=[f+g] .
$$


We can also generalize the Baer-Specker group to the quotient group $\operatorname{Baire}\left(X, \mathbb{Z}^{I}\right) /$ $={ }_{\text {aa }}$. Then $\operatorname{Baire}\left(X, \mathbb{N}^{I}\right) /={ }_{\text {aa }}$ embeds as a monoid in $\operatorname{Baire}\left(X, \mathbb{Z}^{I}\right) /=_{\text {aa }}$, and in particular it is a cancellative monoid.

We use forcing to prove that the quotient is a complete semilattice satisfying all of the distributivity laws of $\mathbb{N}^{I}$.

Theorem 4.40. Let $X$ be a definable Hausdorff and 'complete' topological space (e.g. $X=\mathbb{R})$. Then (Baire $\left.\left(X, \mathbb{N}^{I}\right) /={ }_{\mathrm{aa}},+\right)$ is a commutative cancellative complete semilattice monoid, that is (JID), (MID), infinitely + -distributive over $\vee$ and $\wedge$ and flat-complete. The monoid order is $\leq_{\mathrm{aa}}$, and the lattice operations are given by

$$
[f] \vee[g]=[\max \{f, g\}] \text { and }[f] \wedge[g]=[\min \{f, g\}] .
$$

And we have the forcing expressions

$$
\begin{array}{llll}
\operatorname{Cat}(X) \Vdash \dot{h}=\max \{\dot{f}, \dot{g}\} & \text { iff } & {[h]=[f] \vee[g],} \\
\operatorname{Cat}(X) \Vdash \dot{h}=\min \{\dot{f}, \dot{g}\} & \text { iff } & {[h]=[f] \wedge[g],} \\
\operatorname{Cat}(X) \Vdash \dot{h}=\dot{f}+\dot{g} & \text { iff } & {[h]=[f]+[g] .}
\end{array}
$$

Furthermore, equations (4.38) and (4.39) generalize to families $\mathcal{F} \subseteq \operatorname{Baire}\left(X, \mathbb{N}^{I}\right)$ as

$$
\begin{array}{rlrl}
\operatorname{Cat}(X) \Vdash \dot{h} & =\bigvee_{f \in \mathcal{F}} \dot{f} & \text { iff } & {[h]=\bigvee_{f \in \mathcal{F}}[f],} \\
\operatorname{Cat}(X) \Vdash \dot{h}=\bigwedge_{f \in \mathcal{F}} \dot{f} & \text { iff } & {[h]=\bigwedge_{f \in \mathcal{F}}[f],}
\end{array}
$$

respectively.

Proof. Equation (4.40) follows from the forcing theorem (4.30) with $\varphi\left(x_{0}, x_{1}, x_{2}\right)=$ $\left\ulcorner x_{0}=x_{1}+x_{2}\right\urcorner$. First we verify that $\leq_{\text {aa }}$ coincides with the monoid order. Recall that the monoid ordering of $\mathbb{N}^{I}$ coincides with the usual ordering (example 2.28). Thus using (4.32) and (4.40),

$$
\begin{array}{rll}
f \leq_{\mathrm{aa}} h \quad \text { iff } & \operatorname{Cat}(X) \Vdash \dot{f} \leq \dot{h} \\
& \text { iff } & \operatorname{Cat}(X) \Vdash \exists g \in \mathbb{N}^{I} \dot{h}=\dot{f}+\dot{g} \\
& \text { iff } & \exists g \in \operatorname{Baire}\left(X, \mathbb{N}^{I}\right) \quad \operatorname{Cat}(X) \Vdash \dot{h}=\dot{f}+\dot{g} \\
\text { iff } & \exists g[h]=[f]+[g] \\
\text { iff } & {[f] \leq{ }_{\left(\operatorname{Baire}\left(X, \mathbb{N}^{I}\right) /=_{\mathrm{aa}},+\right)}[h] .}
\end{array}
$$

Next we prove equation (4.38). Recall that max and min are the lattice operations on $\left(\mathbb{N}^{I}, \leq\right)$. Suppose $\operatorname{Cat}(X) \Vdash \dot{h}=\max \{\dot{f}, \dot{g}\}$. Then $[f],[g] \leq_{\text {aa }}[h]$ by (4.32), and if $\left[h^{\prime}\right] \geq$ aa $[f],[g]$ then $\operatorname{Cat}(X) \Vdash \dot{h}^{\prime} \geq \dot{h}$ by (4.32), and thus $[h] \leq_{\text {aa }}\left[h^{\prime}\right]$ again by (4.32), proving $[h]=[f] \vee[g]$. Conversely, suppose $[h]=[f] \vee[g]$. Then $\operatorname{Cat}(X) \Vdash \dot{h} \geq$ $\max \{\dot{f}, \dot{g}\}$ by (4.32). And if $\operatorname{Cat}(X) \Vdash \dot{h}^{\prime} \geq \max \{\dot{f}, \dot{g}\}$, then $\operatorname{Cat}(X) \Vdash \dot{h} \leq \dot{h}^{\prime}$ because $[h] \leq_{\text {aa }}\left[h^{\prime}\right]$, completing the proof that $\operatorname{Cat}(X) \Vdash \dot{h}=\max \{\dot{f}, \dot{g}\}$. The same proof generalizes to arbitrary $\mathcal{F}$ as in equation (4.41). Equations (4.39) and (4.42) 
hold dually. And the lattice operations (4.37) follow from (4.38) and (4.39) and the forcing theorem (4.30), e.g. with $\varphi\left(x_{0}, x_{1}, x_{2}\right)=\left\ulcorner x_{0}=\max \left\{x_{1}, x_{2}\right\}\right.$ ?.

Now we prove that $\operatorname{Baire}\left(X, \mathbb{N}^{I}\right) /={ }_{\text {aa }}$ is a complete semilattice. Suppose $\mathcal{F} \subseteq$ $\operatorname{Baire}\left(X, \mathbb{N}^{I}\right) /=_{\text {aa }}$ is a bounded subfamily. Then letting $\mathcal{F}^{\prime} \subseteq \operatorname{Baire}\left(X, \mathbb{N}^{I}\right)$ be a selection of representatives, $\operatorname{Cat}(X) \Vdash\left\ulcorner\dot{\mathcal{F}}^{\prime}\right.$ is bounded $\urcorner$ by (4.32). Then since $\mathbb{N}^{I}$ is a complete semilattice, there exists $g \in \operatorname{Baire}\left(X, \mathbb{N}^{I}\right)$ such that

$$
\operatorname{Cat}(X) \Vdash \dot{g}=\bigvee \dot{\mathcal{F}}^{\prime}
$$

Thus $[g]=\bigvee \mathcal{F}$ follows from (4.32).

To prove the (JID), suppose $f \in \operatorname{Baire}\left(X, \mathbb{N}^{I}\right), \mathcal{F} \subseteq \operatorname{Baire}\left(X, \mathbb{N}^{I}\right) /={ }_{\text {aa }}$ and $\bigvee \mathcal{F}$ exists. Letting $\mathcal{F}^{\prime} \subseteq \operatorname{Baire}\left(X, \mathbb{N}^{I}\right)$ be a selection of representatives, $\operatorname{Cat}(X) \Vdash \bigvee \dot{\mathcal{F}}^{\prime}$ exists. And then since $\mathbb{N}^{I}$ satisfies the (JID), $\operatorname{Cat}(X) \Vdash \dot{f} \wedge \bigvee \dot{\mathcal{F}}^{\prime}=\bigvee\left(\dot{f} \wedge \dot{\mathcal{F}}^{\prime}\right)$. Therefore, $f \wedge \bigvee \mathcal{F}=\bigvee(f \wedge \mathcal{F})$.

The (MID) property holds dually. And infinite +-distributivity over $\vee$ and $\wedge$ is a consequence of theorem 2.31 (although it can be transferred from $\mathbb{N}^{I}$ similarly to the (JID)). Flat-completeness transfers from $\mathbb{N}^{I}$ similarly, as $\mathbb{N}^{I}$ is flat-complete (example 3.61).

Remark 4.41. Theorem 4.40 can be proved in a routine matter without using forcing, and indeed a drawback of the forcing method is the need for the definable topological requirements on $X$, i.e. the theorem is true for any topological space $X$. However, the reason that it is possible to give a straightforward direct proof of e.g. completeness is that the supremum of a bounded family has a simple definition in terms of the family. Indeed there are very similar situations, involving for example the eventual dominance order $\leq^{*}$ on $\mathbb{N}^{I}$ (see 4 4.6.4) ) rather than the product order, where the supremum has no reasonably simple definition and the forcing translation becomes essential (see e.g. Hir00a]).

Remark 4.42. If the quotient over $\{=\}_{\text {aa }}$ is taken instead, then the quotient lattice over the relation $\{\leq\}_{\text {aa }}$ is not in general a complete semilattice.

Remark 4.43. Note that equation (4.37) does not generalize from two functions to arbitrary families of functions. For a counterexample, consider the family $\left\{f_{z}: z \in\right.$ $X\}$ where $f_{z}(z)=\mathbf{1}_{I}$ and $f_{z}(y)=\mathbf{0}_{I}$ for all $y \neq z$.

Notation 4.44. Let $X$ be some fixed topological space, with largest open meager set $U_{X}$ (cf. proposition 4.17). For $a \in \operatorname{Cat}(X)$, we let $\bar{a}$ denote the regular open set $B \in \operatorname{RO}\left(X \backslash \bar{U}_{X}\right)$ such that $a=[B]$. Let $I$ be some fixed index set. For $a \in \operatorname{Cat}(X)$ and $x \in \mathbb{N}^{I}$ with $x \neq \mathbf{0}_{I}$, we let $\langle a, x\rangle$ denote the member $[f]$ of $\operatorname{Baire}\left(X, \mathbb{N}^{I}\right) /=$ aa where $f: X \rightarrow \mathbb{N}^{I}$ is given by

$$
f(z)= \begin{cases}x & \text { if } z \in \bar{a}, \\ \mathbf{0}_{I} & \text { if } z \notin \bar{a} .\end{cases}
$$

Let $\langle a, x\rangle^{+}$denote $x$. For each $i \in I$, we let $\pi_{i}:\left(\mathbb{N}^{I}\right)^{X} \rightarrow \mathbb{N}^{X}$ denote the pointwise projection

$$
\pi_{i}(f)(z)=f(z)(i) \quad \text { for all } z \in X
$$


Now assume that $S$ is some fixed topological space. Suppose that $\varphi\left(x_{0}, \ldots, x_{n-1}\right.$, $\left.y_{0}, \ldots, y_{m-1}\right)$ is a formula and $a_{0}, \ldots, a_{m-1}$ are some parameters such that $\{z \in X$ : $\left.\varphi\left(f_{0}(z), \ldots, f_{n-1}(z), a_{1}, \ldots, a_{m-1}\right)\right\} \in \mathrm{BP}(X)$ whenever $f_{0}, \ldots, f_{n-1}$ are Baire measurable functions from $X$ into $S$. Then we denote the equivalence class

$$
\left[z \in X: \varphi\left(f_{0}(z), \ldots, f_{n-1}(z), a_{1}, \ldots, a_{m-1}\right)\right] \in \operatorname{Cat}(X) .
$$

by $\left\|\varphi\left(\dot{f}_{0}, \ldots, \dot{f}_{n-1}, a_{1}, \ldots, a_{m-1}\right)\right\|$. In forcing terminology, this is the Boolean truth value of the sentence $\varphi\left(\dot{f}_{0}, \ldots, \dot{f}_{n-1}, a_{0}, \ldots, a_{m-1}\right)$. The following examples illustrate the usefulness of this notation.

Example 4.45. Suppose $f: X \rightarrow \mathbb{N}^{I}$ is Baire measurable. Then for all $i \in I$ and $n \in \mathbb{N}$,

$$
\|\dot{f}(i)=n\|=[z \in X: f(z)(i)=n] .
$$

Suppose $K: X \rightarrow \mathcal{P}(Y)$ is a Baire function where $Y$ is some fixed set. Then for all $y \in Y$,

$$
\|y \in \dot{K}\|=[z \in X: y \in K(z)] .
$$

For $[f] \in \operatorname{Baire}\left(X, \mathbb{N}^{I}\right) /={ }_{\text {aa }}$, we denote

$$
\operatorname{pv}([f])=\left\|\dot{f} \neq \mathbf{0}_{I}\right\| .
$$

We can also write $\pi_{i}([f])$ for $\left[\pi_{i}(f)\right]$ when dealing with equivalence classes modulo $={ }_{\text {aa. }}$. Note that

$$
\operatorname{pv}\left(\pi_{i}([f])\right)=\operatorname{pv}\left(\left\langle 1_{\operatorname{Cat}(X)}, \chi_{i}\right\rangle \wedge[f]\right) .
$$

Also note that

$$
[f]=\bigvee_{i \in I} \bigvee_{n=0}^{\infty} n \cdot\left\langle\|\dot{f}(i)=n\|, \chi_{i}\right\rangle,
$$

because one obtains $[f]=\bigvee_{i \in I} \pi_{i}([f]) \cdot \chi_{i}$ from equation (4.41) and the fact that by equation (4.3),

$$
\operatorname{Cat}(X) \Vdash \dot{f}=\bigvee_{i \in I} \dot{f}(i) \cdot \chi_{i}=\bigvee_{i \in I} \dot{\pi}_{i}(f) \cdot \chi_{i}
$$

(or alternatively use proposition [4.39); and since clearly $\left[\pi_{i}(f) \cdot \chi_{i}\right]=\bigvee_{n=0}^{\infty} n$. $\left\langle\|\dot{f}(i)=n\|, \chi_{i}\right\rangle$.

Definition 4.46. We call members of $\operatorname{Baire}\left(X, \mathbb{N}^{I}\right) /=_{\mathrm{aa}}$ of the form $\left\langle a, n \cdot \chi_{i}\right\rangle$ ( $a \in \operatorname{Cat}(X), n \in \mathbb{N}, i \in I)$ basic elements.

Proposition 4.47. The basic elements form a basis for $\left(\operatorname{Baire}\left(X, \mathbb{N}^{I}\right) /={ }_{\mathrm{aa}}, \leq_{\mathrm{aa}}\right)$.

Proof. By equation (4.52), because $n \cdot\left\langle\|\dot{f}(i)=n\|, \chi_{i}\right\rangle \wedge n^{*} \cdot\left\langle\left\|\dot{f}\left(i^{*}\right)=n^{*}\right\|, \chi_{i^{*}}\right\rangle=[\mathbf{0}]$ whenever either $i \neq i^{*}$ or $n \neq n^{*}$.

Proposition 4.48. (Baire $\left.\left(X, \mathbb{N}^{I}\right) /={ }_{\text {aa }}\right)_{\left\langle a, \chi_{i}\right\rangle} \cong \operatorname{Cat}(X)_{a}$ (cf. notation 3.54).

Proof. $\left\langle b, \chi_{i}\right\rangle \mapsto b$ is an isomorphism. 
We generalize notation 4.9,

Notation 4.49. For a function $K: Y \rightarrow \mathcal{P}(J)$, we let $\mathbf{0}_{K}$ - denote the element of $C\left(\mathbb{N}^{K-}\right)$ given by $\mathbf{0}_{K-}(z)=\mathbf{0}_{K(z)}$ for all $z \in Y$. For a fixed index set $I \subseteq J$, when $Y$ is implicitly understood, we write $\mathbf{0}_{I-}$ for the function in $\left(\mathbb{N}^{I}\right)^{Y}$ constantly equal to $\mathbf{0}_{I}$; in this case, we may write $\mathbf{0}-$ when the index set is also understood.

Theorem 4.50. If $\sigma$ is an order embedding of Baire $\left(X, \mathbb{N}^{I}\right) /={ }_{\text {aa }}$ into Baire $\left(Y, \mathbb{N}^{J}\right) /$ $={ }_{\text {aa }}$ with convex range then $\sigma$ is a dually continuous lattice homomorphism determined by $\sigma\left(\left\langle a, \chi_{i}\right\rangle\right)(a \in \operatorname{Cat}(X), i \in I)$. Indeed it is of the form

$$
\sigma([f])=\bigvee_{i \in I} \bigvee_{n=0}^{\infty} n \cdot \sigma\left(\left\langle\|\dot{f}(i)=n\|, \chi_{i}\right\rangle\right)+[h]
$$

for some $[h] \in \operatorname{Baire}\left(Y, \mathbb{N}^{J}\right) /={ }_{\text {aa }}$. And for each $i \in I, \sigma\left(\left\langle a, \chi_{i}\right\rangle\right)$ is determined by $a_{j}^{i} \in \operatorname{Cat}(X)(j \in J)$ where

$$
\begin{aligned}
a_{j}^{i} \wedge a_{j^{*}}^{i} & =0_{\operatorname{Cat}(X)} \quad \text { for all } j \neq j^{*}, \\
\bigvee_{j \in J} a_{j}^{i} & =1_{\operatorname{Cat}(X)},
\end{aligned}
$$

and isomorphisms $\tau_{j}^{i}: \operatorname{Cat}(X)_{a_{j}^{i}} \rightarrow \operatorname{Cat}(Y)_{\tau\left(a_{j}^{i}\right)}(j \in J)$ via:

$$
\sigma\left(\left\langle a, \chi_{i}\right\rangle\right)=\bigvee_{j \in J}\left\langle\tau_{j}^{i}\left(a \wedge a_{j}^{i}\right), \chi_{j}\right\rangle+[h]
$$

subject to the constraint

$$
\tau_{j}^{i}\left(a_{j}^{i}\right) \wedge \tau_{j}^{i^{*}}\left(a_{j}^{i^{*}}\right)=0_{\mathrm{Cat}(Y)} \text { for all } i \neq i^{*} .
$$

Conversely, any such $\tau_{j}^{i}(i \in I, j \in J)$ and $h$ determine an order embedding $\sigma$ defined by equations (4.54) and (4.57).

Proof. Note that we can assume that $\sigma\left(\left[\mathbf{0}_{I-}\right]\right)=\left[\mathbf{0}_{J-}\right]$ by considering the homomorphism $\sigma^{\prime}=\sigma-\sigma\left(\left[\mathbf{0}_{I-}\right]\right)$ instead. That $\sigma$ is a dually continuous lattice homomorphism is by corollary 3.43 .

Claim 4.51. If $[g]$ is a basic element, say $\left\langle a, n \cdot \chi_{i}\right\rangle$ for some $n, i$ and $a$, then $\sigma([g]) \leq_{\text {aa }}$ n.

Proof. The proof is by induction on $n=0,1,2, \ldots$ For $n=1,2, \ldots$, suppose $[g]=\left\langle a, n \cdot \chi_{i}\right\rangle$ for some $i$ and $a$, and by theorem 4.40 and remark 4.41 we can let $\left[h_{n}\right]$ be the supremum of all $[f] \leq_{\text {aa }}[g]$ such that $\sigma([f]) \leq_{\text {aa }} \mathbf{n}$. By continuity, $\sigma\left(\left[h_{n}\right]\right) \leq_{\text {aa }} \mathbf{n}$. Assuming the claim fails for $g,\left[h_{n}\right]<$ aa $[g]$, and thus there exists a $b_{n} \leq a$ such that

$$
\left[h_{n}\right] \wedge\left\langle b_{n}, n \cdot \chi_{i}\right\rangle=\left\langle b_{n}, k \cdot \chi_{i}\right\rangle
$$

for some $k=0, \ldots, n-1$. Then $\sigma\left(\left\langle b_{n}, n \cdot \chi_{i}\right\rangle\right)$ is not almost always at most $\mathbf{n}$, and thus there is a basic $[p] \leq_{\text {aa }} \sigma\left(\left\langle b_{n}, n \cdot \chi_{i}\right\rangle\right)$ such that $p^{+}=m \cdot \chi_{j}$ for some $m>n$ and some $j$. But then there exists a basic $[q] \leq_{\text {aa }}[p]$ where $q^{+}=n \cdot \chi_{j}$. Letting $[f]$ be the element mapped to $[q],[f] \leq_{\text {aa }}\left\langle b_{n}, n \cdot \chi_{i}\right\rangle$ by order reflection. However, 
$[f] \leq_{\mathrm{aa}}\left[h_{n}\right]$ by the definition of $h_{n}$, and thus $[f] \leq_{\mathrm{aa}}\left\langle b_{n}, k \cdot \chi_{i}\right\rangle$ by (4.59). Now we have obtained a contradiction because by the induction hypothesis $\sigma([f]) \leq_{\text {aa }} \mathbf{k}$, and thus $\sigma([f]) \neq[q]$.

Consider for each $i \in I$ and $j \in J$,

$$
b_{j}^{i}=\operatorname{pv}\left(\left\langle 1_{\operatorname{Cat}(Y)}, \chi_{j}\right\rangle \wedge \sigma\left(\left\langle 1_{\operatorname{Cat}(X)}, \chi_{i}\right\rangle\right)\right)=\operatorname{pv}\left(\pi_{j}\left(\sigma\left(\left\langle 1_{\operatorname{Cat}(X)}, \chi_{i}\right\rangle\right)\right)\right) .
$$

Since $\sigma$ is injective and $\operatorname{ran}(\sigma)$ is downwards closed, there is a unique $a_{j}^{i} \in \operatorname{Cat}(X)$ such that

$$
\sigma\left(\left\langle a_{j}^{i}, \chi_{i}\right\rangle\right)=\left\langle b_{j}^{i}, \chi_{j}\right\rangle,
$$

and thus by proposition 4.48 we can define an isomorphism $\tau_{j}^{i}: \operatorname{Cat}(X)_{a_{j}^{i}} \rightarrow$ $\operatorname{Cat}(Y)_{b_{j}^{i}}$ by

$$
\sigma\left(\left\langle a, \chi_{i}\right\rangle\right)=\left\langle\tau_{j}^{i}(a), \chi_{j}\right\rangle .
$$

Now since claim 4.51 implies that $\sigma\left(\left\langle 1_{\mathrm{Cat}(X)}, \chi_{i}\right\rangle\right) \leq_{\text {aa }} \mathbf{1}$, equation (4.61) implies equation (4.56). And then equation (4.57), with $h=\mathbf{0}-$, follows from (4.56), (4.62) and continuity. Note too that (4.55) holds because $\left\langle b_{j}, \chi_{j}\right\rangle \wedge\left\langle b_{j^{*}}, \chi_{j^{*}}\right\rangle$ for $j \neq j^{*}$.

Claim 4.52. For all $g, \operatorname{pv}\left(\pi_{j}(\sigma([g]))\right)=\operatorname{pv}\left(\pi_{j}(\sigma([n \cdot g]))\right)$ for all $n=1,2, \ldots$, and all $j \in J$.

Proof. Since $\sigma$ is order preserving and $n \geq 1, \sigma([g]) \leq \sigma([n \cdot g])$. It thusly suffices to show that $\operatorname{pv}\left(\pi_{j}(\sigma([n \cdot g]))\right)-\operatorname{pv}\left(\pi_{j}(\sigma([g]))\right)=0_{\operatorname{Cat}(Y)}$. Supposing to the contrary that the difference $a$ is nonzero, then $\left\langle a, \chi_{j}\right\rangle \leq_{\text {aa }} \sigma([n \cdot g])$. And then there is an $[f] \leq_{\text {aa }}[n \cdot g]$ such that $\sigma([f])=\left\langle a, \chi_{j}\right\rangle$. However, $[f] \wedge[g] \neq[\mathbf{0}-]$ and thus $\sigma([f]) \wedge \sigma([g])={ }_{\text {aa }} \sigma([f] \wedge[g]) \neq[\mathbf{0}-]$, contradicting the fact that $\left\langle a, \chi_{j}\right\rangle \wedge \sigma([g])=$ $[\mathbf{0}-]$.

This establishes by induction on $n=0,1,2, \ldots$ that

$$
\sigma\left(\left\langle a, n \cdot \chi_{i}\right\rangle\right)=n \cdot \sigma\left(\left\langle a, \chi_{i}\right\rangle\right) .
$$

To see this, first note that by continuity and (4.56), we may assume that $a \leq a_{j}^{i}$ for some $j$. By claim 4.51, $\sigma\left(\left\langle a, n \cdot \chi_{i}\right\rangle\right) \leq_{\text {aa }} \mathbf{n}$, while by (4.62) and claim 4.52, $\operatorname{pv}\left(\pi_{j^{*}}\left(\sigma\left(\left\langle a, n \cdot \chi_{i}\right\rangle\right)\right)\right)=0_{\text {Cat }(Y)}$ for all $j^{*} \neq j$, and $\operatorname{pv}\left(\pi_{j}\left(\sigma\left(\left\langle a, n \cdot \chi_{i}\right\rangle\right)\right)\right)=\tau_{j}^{i}(a)$, and therefore $\sigma\left(\left\langle a, n \cdot \chi_{i}\right\rangle\right) \leq_{\text {aa }}\left\langle\tau_{j}^{i}(a), n \cdot \chi_{j}\right\rangle=n \cdot \sigma\left(\left\langle a, \chi_{i}\right\rangle\right)$.

As for the opposite inequality, supposing towards a contradiction that $\sigma(\langle a, n$. $\left.\left.\chi_{i}\right\rangle\right)<_{\text {aa }}\left\langle\tau_{j}^{i}(a), n \cdot \chi_{j}\right\rangle$, there exists $b \leq \tau_{j}^{i}(a)$ such that

$$
\sigma\left(\left\langle a, n \cdot \chi_{i}\right\rangle\right) \wedge\left\langle b, n \cdot \chi_{j}\right\rangle={ }_{\text {aa }}\left\langle b, k \cdot \chi_{j}\right\rangle
$$

for some $k=0, \ldots, n-1$. Then letting $c$ be the element such that $\tau_{j}^{i}(c)=b$, $\sigma\left(\left\langle c, k \cdot \chi_{i}\right\rangle\right)=k \cdot \sigma\left(\left\langle c, \chi_{i}\right\rangle\right)=k \cdot\left\langle b, \chi_{j}\right\rangle=\left\langle b, k \cdot \chi_{j}\right\rangle$ by the induction hypothesis; however, $\sigma\left(\left\langle c, n \cdot \chi_{i}\right\rangle\right) \leq_{\text {aa }} \sigma\left(\left\langle a, n \cdot \chi_{i}\right\rangle\right)$ and we know that $\sigma\left(\left\langle c, n \cdot \chi_{i}\right\rangle\right) \leq_{\text {aa }}\left\langle b, n \cdot \chi_{j}\right\rangle$ because we have proved the other inequality of (4.63). Thus $\sigma\left(\left\langle c, n \cdot \chi_{i}\right\rangle\right) \leq_{\text {aa }}\left\langle b, k \cdot \chi_{j}\right\rangle$ by (4.64), contradicting the fact that $\sigma$ is a monomorphism.

Equations (4.52) and (4.63) and continuity establish (4.54), and (4.58) easily follows from the injectivity of $\sigma$. 
The converse should be clear.

Corollary 4.53. Order isomorphisms between Baire $\left(\mathbb{N}^{I}\right) /={ }_{\text {aa }}$ and Baire $\left(\mathbb{N}^{J}\right) /={ }_{\text {aa }}$ are precisely those homomorphisms satisfying (4.54)-(4.58), $[h]=\left[\mathbf{0}_{J-}\right]$ and the equation

$$
\bigvee_{i \in I} \tau_{j}^{i}\left(a_{j}^{i}\right)=1_{\operatorname{Cat}(Y)} \quad \text { for all } j \in J,
$$

or equivalently $\bigwedge_{j \in J} \bigvee_{i \in I} \tau_{j}^{i}\left(a_{j}^{i}\right)=1_{\mathrm{Cat}(Y)}$.

We would like to generalize theorem 4.10 to the realm of Baire functions. We begin by imposing topological requirements to apply the results of $\$ 4.3$, in order to obtain a preliminary result (corollary 4.55).

Notation 4.54. In the context of notation 4.44, we remove the dot when we do not want to go to the equivalence class modulo $\mathcal{M}(X)$, and thus $\| \varphi\left(f_{0}, \ldots, f_{n-1}, a_{0}, \ldots\right.$, $\left.a_{m-1}\right) \|=\left\{z \in X: \varphi\left(f_{0}(z), \ldots, f_{n-1}(z), a_{0}, \ldots, a_{m-1}\right)\right\}$. Thus for example, for any $f: X \rightarrow \mathbb{N}^{I},\|f(i)=n\|=\{z \in X: f(z)(i)=n\}$. Also, when we take a subset $B \subseteq X$ as opposed to a member of $\operatorname{Cat}(X)$, the notation $\langle B, x\rangle\left(x \in \mathbb{N}^{I}\right)$ refers to the function $f: X \rightarrow \mathbb{N}^{I}$ such that $f(z)=x$ for $z \in B$, and $f(z)=\mathbf{0}_{I-}$ when $x \notin B$.

Corollary 4.55. Let $X$ be a regular space, and suppose $Y$ has a Baire compactifcation. Order embeddings $\sigma: \operatorname{Baire}\left(X, \mathbb{N}^{I}\right) /={ }_{\text {aa }} \rightarrow \operatorname{Baire}\left(Y, \mathbb{N}^{J}\right) /=_{\text {aa }}$ with convex range are completely determined by $B_{j} \in \mathrm{BP}(Y), H_{j} \in \operatorname{Baire}\left(B_{j}, X\right)$ such that

$$
\begin{gathered}
H_{j}^{-1}[[\mathcal{M}(X)]] \subseteq \mathcal{M}(Y), \\
H_{j}^{-1}[M] \cap\left\|g_{j}=i\right\| \in \mathcal{M}(Y) \quad \text { implies } \quad M \cap H_{j}\left[\left\|g_{j}=i\right\|\right] \in \mathcal{M}(X)
\end{gathered}
$$

for every $i \in I$ and $M \subseteq X$,

$$
\begin{gathered}
H_{j}\left[\left\|g_{j}=i\right\|\right] \cap H_{j^{*}}\left[\left\|g_{j^{*}}=i\right\|\right]=\emptyset \quad \text { for all } j \neq j^{*} \text { in } J, \text { and all } i \in I, \\
\bigvee_{j \in J}\left[H_{j}\left[\left\|g_{j}=i\right\|\right]\right]_{\operatorname{Cat}(X)}=1_{\operatorname{Cat}(X)} \quad \text { for all } i \in I,
\end{gathered}
$$

and $g_{j} \in C\left(B_{j}, I\right)(j \in J)$ via $\sigma([f])=\left[\rho_{f}\right]+\sigma\left(\left[\mathbf{0}_{I-}\right]\right)$ where

$$
\rho_{f}(z)(j)= \begin{cases}f\left(H_{j}(z)\right)\left(g_{j}(z)\right) & z \in B_{j}, \\ 0 & z \notin B_{j},\end{cases}
$$

for all $j \in J$.

Proof. Let $a_{j}^{i}$ and $\tau_{j}^{i}(i \in I, j \in J)$ be as given to us by theorem 4.50, For each $i$ and $j$, by corollary 4.31, there exists a relatively comeager $C_{j}^{i} \subseteq \bar{\tau}_{j}^{i}\left(a_{j}^{i}\right)$ and a Baire measurable injection $f_{j}^{i}: C_{j}^{i} \rightarrow \bar{a}_{j}^{i}$ satisfying

$$
\tau_{j}^{i}([B])=\left[\left(f_{j}^{i}\right)^{-1}[B]\right] \text { for all } B \in \operatorname{BP}\left(\bar{a}_{j}^{i}\right)
$$

and conditions (国)-(ㄷ) of the conclusion of the corollary (note that $A=\emptyset$ there since $\tau_{j}^{i}$ is an isomorphism). Note that $\bar{\tau}_{j}^{i}\left(a_{j}^{i}\right) \cap \bar{\tau}_{j}^{i^{*}}\left(a_{j}^{i^{*}}\right)=\emptyset$ for all $i \neq i^{*}$ by (4.58). 
For each $j \in J$, put $B_{j}=\bigcup_{i \in I} C_{j}^{i}$, and note that $B_{j} \in \mathrm{BP}(Y)$ by disjointness and proposition 4.16. Define $g_{j}: B_{j} \rightarrow I$ by

$$
g_{j}(z)=\text { the unique } i \in I \text { such that } z \in C_{j}^{i} \text {. }
$$

Then define $H_{j}: B_{j} \rightarrow X$ by

$$
H_{j}(z)=f_{j}^{g_{j}(z)}(z)
$$

Then $H_{j}$ is Baire measurable since each $f_{j}^{i}$ is and by disjointness. Noting that $\left\|g_{j}=i\right\|=C_{j}^{i}$, equations (4.66) and (4.67) hold by condition (b). Equation (4.68) holds by (4.55), because each $H_{j}\left[\left\|g_{j}=i\right\|\right]=\operatorname{ran}\left(f_{j}^{i}\right) \subseteq \bar{a}_{j}^{i}$. And equation (4.69) holds by (4.56), because each $\left[H_{j}\left[\left\|g_{j}=i\right\|\right]\right]_{\operatorname{Cat}(X)}=\left[\operatorname{ran}\left(f_{j}^{i}\right)\right]_{\operatorname{Cat}(X)}=a_{j}^{i}$.

Claim 4.56. $f \leq_{\text {aa }} f^{\prime}$ implies $\rho_{f} \leq_{\text {aa }} \rho_{f^{\prime}}$.

Proof. Fixing $j \in J$, we must show that $\rho_{f}(z)(j) \leq \rho_{f^{\prime}}(z)(j)$ for almost all $z \in Y$. Now $\left\{z: \rho_{f}(z)(j) \not \leq \rho_{f^{\prime}}(z)(j)\right\}=\bigcup_{i \in I} D_{i}$ where $D_{i}=\left\{z \in C_{j}^{i}: f\left(H_{j}(z)\right)(i) \not \leq\right.$ $\left.f^{\prime}\left(H_{j}(z)\right)(i)\right\}$. And it follows from equation (4.66) that each of the $D_{i}$ 's is meager. But $\left\{C_{j}^{i}: i \in I\right\}$ is a pairwise disjoint family of relatively open subsets of $B_{j}$, and thus $\bigcup_{i \in I} D_{i}$ is meager by proposition 4.16 .

The preceding claim entails that $f={ }_{\text {aa }} f^{\prime}$ implies $\rho_{f}={ }_{\text {aa }} \rho_{f^{\prime}}$, and thus $[f] \mapsto\left[\rho_{f}\right]$ is well defined. Also note that indeed $\rho_{f}$ is Baire measurable: By lemma 4.36 there is a Borel $f^{\prime}=$ aa $f$, and $f^{\prime} \circ H$ is thus Baire measurable being the composition of a Borel function with a Baire function. The Baire measurability of $\rho_{f^{\prime}}$ now easily follows from the continuity of the $g_{j}$ 's. Hence $\rho_{f}=$ aa $\rho_{f^{\prime}}$ implies $\rho_{f} \in \operatorname{Baire}\left(Y, \mathbb{N}^{J}\right)$ by proposition 4.37 .

Claim 4.57. Whenever $\mathcal{F} \subseteq \operatorname{Baire}\left(X, \mathbb{N}^{I}\right) /=$ aa has a supremum $\left[f^{\prime}\right], \bigvee_{[f] \in \mathcal{F}}\left[\rho_{f}\right]=$ $\left[\rho_{f^{\prime}}\right]$.

Proof. Let $\left[f^{\prime}\right]=\bigvee \mathcal{F}$. Then $\left[\rho_{f}\right] \leq_{\text {aa }}\left[\rho_{f^{\prime}}\right]$ for all $[f] \in \mathcal{F}$, by claim 4.56. And $\left[\rho_{f^{\prime}}\right]$ is moreover the least upper bound: For if $\rho_{f^{\prime}} \Varangle_{\text {aa }} h^{\prime}$ then there exists $j \in J$ such that not almost all $z \in Y$ satisfy $\rho_{f^{\prime}}(z)(j) \leq h^{\prime}(z)(j)$. And then there is an $i \in I$ such that $C=\left\{z \in C_{j}^{i}: \rho_{f^{\prime}}(z)(j) \not \leq h^{\prime}(z)(j)\right\} \notin \mathcal{M}(Y)$. And we can find a nonmeager $D \subseteq C$ and $n \in \mathbb{N}$ such that $h^{\prime}(z)(j)=n$ for all $z \in D$. Since $g_{j}(z)=i$ and $H_{j}(z)=f_{j}^{i}(z)$ for all $z \in C_{j}^{i}$, we have

$$
f^{\prime}\left(f_{j}^{i}(z)\right)(i)=\rho_{f^{\prime}}(z)(j)>n \quad \text { for all } z \in D .
$$

Note that condition (b) for $f_{j}^{i}$ implies that $f_{j}^{i}[D] \notin \mathcal{M}(X)$. Therefore, there exists $[f] \in \mathcal{F}$ such that

$$
W=\left\{y \in f_{j}^{i}[D]: f(y)(i)>n\right\} \notin \mathcal{M}(X) .
$$

Now $\rho_{f}(z)(j)=f\left(f_{j}^{i}(z)\right)(i)>n=h^{\prime}(z)(j)$ for all $z \in\left(f_{j}^{i}\right)^{-1}[W]$, as $\left(f_{j}^{i}\right)^{-1}[W] \subseteq D$ since $f_{j}^{i}$ is an injection. Thus $\rho_{f} \Varangle_{\text {aa }} h^{\prime}$ because $\left(f_{j}^{i}\right)^{-1}[W] \notin \mathcal{M}(Y)$ by condition (b) . 
Sublemma 4.58. $\left[\rho_{f}\right]=\bigvee \bigvee_{i \in I} \bigvee_{n=0}^{\infty} n \cdot\left[\rho_{\left\langle\|f(i)=n\|, \chi_{i}\right\rangle}\right]$

Proof. Note that

$$
\rho_{f+f^{\prime}}=\rho_{f}+\rho_{f^{\prime}} .
$$

Thus $\rho_{n \cdot f}=n \cdot \rho_{f}$, and this establishes the sublemma because $\left[\rho_{f}\right]=\bigvee_{i \in I} \bigvee_{n=0}^{\infty}$ $\left[\rho_{\left\langle\|f(i)=n\|, n \cdot \chi_{i}\right\rangle}\right]$ by equation (4.52) and claim 4.57.

Assume without loss of generality that $\sigma\left(\left[\mathbf{0}_{I-}\right]\right)=\left[\mathbf{0}_{J_{-}}\right]$. To verify that $\sigma([f])=$ $\left[\rho_{f}\right]$ for all $[f]$, it suffices by sublemma 4.58 and theorem 4.50 to take $[f]$ of the form $\left\langle a, \chi_{i}\right\rangle$ for some $a \leq a_{j}^{i}, i \in I$ and $j \in J$. Let $f$ be the representative as in (4.45). Then $\rho_{f}(z)\left(j^{*}\right)=0$ unless $g_{j^{*}}(z)=i$ and $H_{j^{*}}(z) \in \bar{a}$, in which case $\rho_{f}(z)\left(j^{*}\right)=1$. Note that $g_{j^{*}}(z)=i$ implies $H_{j^{*}}(z)=f_{j^{*}}^{i}(z) \in \bar{a}_{j^{*}}^{i}$, and thus $H_{j^{*}}(z) \in \bar{a} \subseteq \bar{a}_{j}^{i}$ implies $j^{*}=j$ by (4.55). Therefore, $\left[\rho_{f}\right]=\left\langle\left[\left(f_{j}^{i}\right)^{-1}[\bar{a}]\right], \chi_{j}\right\rangle=\left\langle\tau_{j}^{i}(a), \chi_{j}\right\rangle$ by (4.71). Now by (4.57), we have shown $\left[\rho_{f}\right]=\sigma([f])$.

Remark 4.59. For second countable spaces we can improve to open and continuous $H_{j}(j \in J)$. More precisely, if $X$ is a second countable Hausdorff space and $Y$ is Polish, then we can obtain the result of corollary 4.55 such that the $H_{j}: B_{j} \rightarrow X$ are moreover open and continuous. The proof is the same as for corollary 4.55, but with the stronger hypothesis we can use corollary 4.33 instead of corollary 4.31 to obtain topological embeddings $f_{j}^{i}: B_{j}^{i} \rightarrow \bar{\tau}_{j}^{i}\left(a_{j}^{i}\right)$ for relatively comeager $B_{j}^{i} \subseteq \bar{a}_{j}^{i}$ $(i \in I, j \in J)$. Now we let $C_{j}^{i}=\operatorname{ran}\left(f_{j}^{i}\right)$. Then $H_{j}$ is defined instead by

$$
H_{j}(z)=\left(f_{j}^{g_{j}(z)}\right)^{-1}(z)
$$

and is in fact open and continuous. The rest of the proof goes through the same.

Next we want to generalize the notion of a projection by a function (cf. definition 4.7). This requires a generalization of functions with codomain a power of $\mathbb{N}$.

Notation 4.60. Let $J$ be an index set, and let $X$ and $Y$ be topological spaces. Suppose $K: Y \rightarrow \mathcal{P}(J)$. Then $X^{K-}$ denotes the function $z \mapsto X^{K(z)}$, and thus $\prod X^{K-}\left(=\prod_{z \in Y} X^{K(z)}\right)$ denotes the family of all functions $f$ with domain $Y$ such that

$$
f(z) \in X^{K(z)} \quad \text { for all } z \in Y .
$$

Suppose $f \in \prod X^{K-}$. For each $j \in J$, we let $f^{j}$ denote the function

$$
f^{j}:\|j \in K\| \rightarrow X
$$

where $f^{j}(z)=f(z)(j)$ for all $z \in\|j \in K\|$. We let Baire $\left(X^{K-}\right)$ denote the family of all $f \in \prod X^{K-}$ such that $f^{j} \in \operatorname{Baire}(\|j \in K\|, X)$ for all $j \in J$, and we let $C\left(X^{K-}\right)$ denote the family of all $f \in \prod X^{K-}$ such that $f^{j} \in C(\|j \in K\|, X)$ for all $j \in J$.

Note that this notation is of course a generalization, i.e. if $I \subseteq J$ is some fixed index set then letting $K$ be the function on $Y$ constantly equal to $I, \prod X^{K-}=\left(X^{I}\right)^{Y}$. 
Definition 4.61. Fix index sets $I$ and $J$. For each function $K: Y \rightarrow \mathcal{P}(J)$, and each $g \in \prod I^{K-}$ and $H \in \prod X^{K-}$, we define $\pi_{g, H}:\left(\mathbb{N}^{I}\right)^{X} \rightarrow \prod \mathbb{N}^{K-}$ by

$$
\pi_{g, H}(f)(z)(j)=f(H(z)(j))(g(z)(j)) \text { for all } j \in K(z) \text {, for all } z \in Y \text {. }
$$

Note that this is indeed a generalization of the projections. For if $f \in\left(\mathbb{N}^{I}\right)^{X}$ is constant with respect to $z \in X$, say $f(z)=x$, then $H$ is irrelevant and

$$
\pi_{g, H}(f)(z)=x \circ g(z) \quad \text { for all } z \in Y .
$$

Theorem 4.62. Let $X$ be a regular space and suppose $Y$ has a Baire compactification. The order embeddings from $\operatorname{Baire}\left(X, \mathbb{N}^{I}\right) /={ }_{\text {aa }}$ into $\operatorname{Baire}\left(Y, \mathbb{N}^{J}\right) /={ }_{\text {aa }}$ with convex range consist of maps of the form

$$
\sigma([f])=\left[\pi_{g, H}(f) \boldsymbol{0}_{J \backslash K-}\right]+[h] \text { for all }[f] \in \operatorname{Baire}\left(X, \mathbb{N}^{I}\right) /=_{\text {aa }}
$$

for some $K \in \operatorname{Baire}(Y, \mathcal{P}(J)), g \in C\left(I^{K-}\right), H \in \operatorname{Baire}\left(X^{K-}\right)$ such that

$$
\begin{gathered}
\left(H^{j}\right)^{-1}[M] \in \mathcal{M}(Y) \quad \text { for all } M \in \mathcal{M}(X) \text { and all } j \in J, \\
\left(H^{j}\right)^{-1}[M] \cap\left\|g^{j}=i\right\| \in \mathcal{M}(Y) \quad \text { implies } \quad M \cap H^{j}\left[\left\|g^{j}=i\right\|\right] \in \mathcal{M}(X)
\end{gathered}
$$

for every $i \in I$ and $M \subseteq X$,

$$
\begin{gathered}
H^{j}\left[\left\|g^{j}=i\right\|\right] \cap H^{j^{*}}\left[\left\|g^{j^{*}}=i\right\|\right]=\emptyset \quad \text { for all } j \neq j^{*} \text { in } J, \text { and all } i \in I, \\
\bigvee_{j \in J}\left[H^{j}\left[\left\|g^{j}=i\right\|\right]\right]_{\operatorname{Cat}(X)}=1_{\operatorname{Cat}(X)} \quad \text { for all } i \in I,
\end{gathered}
$$

and some $[h] \in \operatorname{Baire}\left(Y, \mathbb{N}^{J}\right) /={ }_{\mathrm{aa}}$. Moreover, the range is given by

$$
\operatorname{ran}(\sigma)=\left(\operatorname{Baire}\left(\mathbb{N}^{K-}\right) \frown \mathbf{0}_{J \backslash K-}+h\right) /=\text { aa. }
$$

Proof. Let $\sigma$ be such an order embedding with convex range. Let $B_{j} \in \mathrm{BP}(Y)$, $H_{j} \in \operatorname{Baire}\left(B_{j}, X\right), g_{j} \in C\left(B_{j}, I\right)(j \in J)$ and $\rho$ be as given by corollary 4.55. Define $K: Y \rightarrow \mathcal{P}(J)$ by

$$
K(z)=\left\{j \in J: z \in B_{j}\right\} .
$$

Clearly $K \in \operatorname{Baire}(Y, \mathcal{P}(J))$. Define $g \in C\left(I^{K-}\right)$ by

$$
g(z)(j)=g_{j}(z) \text { for all } j \in K(z), \text { for all } z \in Y,
$$

so that $g^{j}=g_{j}$ for all $j \in J$. And we can define $H \in \operatorname{Baire}\left(X^{K-}\right)$ by $H(z)(j)=$ $H_{j}(z)$ for all $j \in K(z)$ and all $z \in Y$, so that $H^{j}=H_{j}$ for all $j$. Equations (4.83)(4.86) will be satisfied by (4.66) -(4.69), respectively. Then

$$
\rho_{f}(z)(j)=f\left(H_{j}(z)\right)\left(g_{j}(z)\right)=f(H(z)(j))(g(z)(j)) \text { for all } j \in K(z),
$$

and $\rho_{f}(z)(j)=0$ for all $j \in J \backslash K(z)$. Hence $\rho_{f}=\pi_{g, H} \frown \mathbf{0}_{J \backslash K-}$, and therefore, $\sigma([f])=\left[\pi_{g, H}(f) \frown \mathbf{0}_{J \backslash K-}\right]+\sigma\left(\left[\mathbf{0}_{I-}\right]\right)$ for all $[f] \in \operatorname{Baire}\left(X, \mathbb{N}^{I}\right) /=$ aa.

Conversely, suppose we are given $K, g, H$ and $h$ as in the corollary. Let $\sigma$ : $\operatorname{Baire}\left(X, \mathbb{N}^{I}\right) /={ }_{\mathrm{aa}} \rightarrow \operatorname{Baire}\left(Y, \mathbb{N}^{J}\right) /=_{\mathrm{aa}}$ be as defined in (4.82). Equation (4.83) and the continuity of the $g^{j}$ s allows us to use the same argument as in the proof of claim 4.56 to prove that $f \leq_{\text {aa }} f^{\prime}$ implies $\pi_{g, H}(f) \leq_{\text {aa }} \pi_{g, H}\left(f^{\prime}\right)$, using $\left\{\left(g^{j}\right)^{-1}: i \in I\right\}$ here in place of $\left\{C_{j}^{i}: i \in I\right\}$ there. This establishes that $\sigma$ is a well defined partial 
order homomorphism. To prove that $\sigma$ is an embedding, it remains to show that $\pi_{g, H}$ is order reflecting. Suppose then that $f \not \mathbb{a a a} f^{\prime}$. There is an $i \in I$ such that not almost all $y \in X$ satisfy $f(y)(i) \leq f^{\prime}(y)(i)$. Thus by (4.86), there exists $j \in J$ such that

$$
\left\{y \in X: f(y)(i)>f^{\prime}(y)(i)\right\} \cap H^{j}\left[\left\|g^{j}=i\right\|\right] \notin \mathcal{M}(X) .
$$

Therefore, $C=\left(H^{j}\right)^{-1}\left[y: f(y)(i)>f^{\prime}(y)(i)\right] \cap\left\|g^{j}=i\right\| \notin \mathcal{M}(Y)$ by equation (4.84). However, for every $z \in C, \pi_{g, H}(f)(z)(j)=f\left(H^{j}(z)\right)\left(g^{j}(z)\right)=f\left(H^{j}(z)\right)(i)>$ $f^{\prime}\left(H^{j}(z)\right)(i)=\pi_{g, H}\left(f^{\prime}\right)(z)(j)$, proving $\pi_{g, H}(f) \not$ aa $_{\text {aa }} \pi_{g, H}\left(f^{\prime}\right)$ as required.

For the converse, we still have yet to show that $\operatorname{ran}(\sigma)$ is convex. However, since

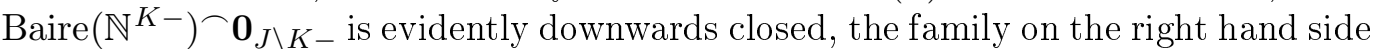
of equation (4.87) is convex. Thus it will suffice to establish (4.87). Without loss of generality we may assume that $[h]=[\mathbf{0}-]$. Take $h^{\prime} \in \operatorname{Baire}\left(\mathbb{N}^{K-}\right)$. Using (4.85) , it is possible to find $f: X \rightarrow \mathbb{N}^{I}$ such that for each $i \in I$,

$$
f\left(H^{j}(z)\right)(i)=h^{\prime}(z)(j) \quad \text { for all } z \in\left\|g^{j}=i\right\|,
$$

for all $j \in J$. Note that (4.86) guarantees that $f$ is Baire measurable. Since each $g^{j}$ is continuous, and since equation (4.92) entails that $\pi_{g, H}(f)(z)(j)=f\left(H^{j}(z)\right)(i)=$ $h^{\prime}(z)(j)$ for almost all $z \in\left\|g^{j}=i\right\|$, for all $i \in I$, we obtain $\pi_{g, H}(f)={ }_{\text {aa }} h^{\prime}$ as desired.

Remark 4.63. When $X$ is a second countable Hausdorff space and $Y$ is Polish, we can moreover obtain $H \in C\left(X^{K-}\right)$ with $H^{j}$ an open continuous mapping for all $j \in J$, by remark 4.59 .

Corollary 4.64. Let $X$ be regular and let $Y$ be a space with a Baire compactification. Any embedding $\sigma$ of $\operatorname{Baire}\left(X, \mathbb{N}^{I}\right) /={ }_{\text {aa }}$ into a downwards closed subset of $\operatorname{Baire}\left(Y, \mathbb{N}^{J}\right) /={ }_{\text {aa }}$ is of the form

$$
\sigma([f])=\left[\pi_{g, H}(f) \frown \mathbf{0}_{J \backslash K-}\right]
$$

for some $K \in \operatorname{Baire}(Y, \mathcal{P}(J)), g \in C\left(I^{K-}\right)$ and $H \in \operatorname{Baire}\left(X^{K-}\right)$. It is thus a monoid homomorphism. Moreover, its range is the equivalence classes of the collection of functions Baire $\left(\mathbb{N}^{K-}\right) \frown \mathbf{0}_{J \backslash K-}$.

Proof. That order embeddings with downwards closed ranges have the indicated form and the indicated range, is an immediate consequence of theorem 4.62 since a downwards closed range implies $[h]=[\mathbf{0}-]$. As for being a monoid homomorphism, observe that

$$
\pi_{g, H}\left(f+f^{\prime}\right)(z)=\pi_{g, H}(f)(z)+\pi_{g, H}\left(f^{\prime}\right)(z) \text { for all } z .
$$

Corollary 4.65. Let $X$ be regular and let $Y$ be a space with a Baire compactification. The order isomorphisms between $\operatorname{Baire}\left(X, \mathbb{N}^{I}\right) /={ }_{\mathrm{aa}}$ and $\operatorname{Baire}\left(Y, \mathbb{N}^{J}\right) /={ }_{\mathrm{aa}}$ consist of maps of the form

$$
[f] \mapsto\left[\pi_{g, H}(f)\right]
$$


for some $g \in C\left(Y, I^{J}\right)$ and $H \in \operatorname{Baire}\left(Y, X^{J}\right)$ such that

$$
\begin{gathered}
\left(H^{j}\right)^{-1}[M] \in \mathcal{M}(Y) \quad \text { for all } M \in \mathcal{M}(X) \text { and all } j \in J, \\
\left(H^{j}\right)^{-1}[M] \cap\left\|g^{j}=i\right\| \in \mathcal{M}(Y) \quad \text { implies } \quad M \cap H^{j}\left[\left\|g^{j}=i\right\|\right] \in \mathcal{M}(X)
\end{gathered}
$$

for every $i \in I$ and $M \subseteq X$,

$$
\begin{gathered}
H^{j}\left[\left\|g^{j}=i\right\|\right] \cap H^{j^{*}}\left[\left\|g^{j^{*}}=i\right\|\right]=\emptyset \quad \text { for all } j \neq j^{*} \text { in } J, \text { and all } i \in I, \\
\bigvee_{j \in J}\left[H^{j}\left[\left\|g^{j}=i\right\|\right]\right]_{\operatorname{Cat}(X)}=1_{\operatorname{Cat}(X)} \quad \text { for all } i \in I .
\end{gathered}
$$

Proof. $g$ and $H$ are obtained from theorem 4.62. Corollary 4.64 tells us that $h$ vanishes, and that $K(z)=J$ for almost all $z$. The function $g$ is continuous because $K$ is constant and the $g^{j}$ 's are continuous.

4.5. Continuous functions into powers of $\mathbb{N}$. For any topological space $X$, $C\left(X, \mathbb{N}^{I}\right) \subseteq \operatorname{Baire}\left(X, \mathbb{N}^{I}\right)$. It is in fact a sublattice, i.e. it is closed under the lattice operations on Baire $\left(X, \mathbb{N}^{I}\right)$, of pointwise max and min (cf. equation (4.35) ).

Proposition 4.66. $C\left(X, \mathbb{N}^{I}\right)$ is a sublattice of $\left(\operatorname{Baire}\left(X, \mathbb{N}^{I}\right), \leq\right)$.

Proof. Take $f, g \in C\left(X, \mathbb{N}^{I}\right)$. The coordinatewise mappings max, min $: \mathbb{N}^{I} \times \mathbb{N}^{I} \rightarrow$ $\mathbb{N}^{I}$ (cf. \$4.2) are both continuous, and $(f, g): X \rightarrow \mathbb{N}^{I} \times \mathbb{N}^{I}$ is continuous. Therefore, $\max \{f, g\}=\max \circ(f, g)$ and $\min \{f, g\}=\min \circ(f, g)$ are both continuous.

Furthermore, $C\left(X, \mathbb{N}^{I}\right)$ is a submonoid of $\left(\operatorname{Baire}\left(X, \mathbb{N}^{I}\right),+\right)$. In fact, noting that addition and subtraction are continuous operations on $\mathbb{Z}^{I}, C\left(X, \mathbb{Z}^{I}\right)$ is closed under subtraction as in the proof of proposition 4.66, and therefore is a subgroup of the Abelian group (Baire $\left(X, \mathbb{Z}^{I}\right),+$ ). Similarly:

Proposition 4.67. $C\left(X, \mathbb{N}^{I}\right)$ is a submonoid of $\left(\operatorname{Baire}\left(X, \mathbb{N}^{I}\right),+\right)$ closed under subtraction.

Thus $C\left(X, \mathbb{N}^{I}\right)$ is essentially a 'subgroup' of $\left(\operatorname{Baire}\left(X, \mathbb{N}^{I}\right),+\right.$ ) (see proposition 1.2); indeed, note that $\left\langle C\left(X, \mathbb{N}^{I}\right)\right\rangle=C\left(X, \mathbb{Z}^{I}\right)$.

Not only are members of $C\left(X, \mathbb{N}^{I}\right)$ representatives of members of Baire $\left(X, \mathbb{N}^{I}\right) /$ $={ }_{\text {aa }}$, but when $X$ is a Baire space, $C\left(X, \mathbb{N}^{I}\right)$ embeds into $\operatorname{Baire}\left(X, \mathbb{N}^{I}\right) /=$ aa.

Proposition 4.68. If $X$ is a Baire space, then $f \mapsto[f]$ is an order embedding of $\left(C\left(X, \mathbb{N}^{I}\right), \leq\right)$ into $\left(\operatorname{Baire}\left(X, \mathbb{N}^{I}\right) /={ }_{\mathrm{aa}}, \leq \mathrm{aa}\right)$, and it is also both a monoid and $a$ lattice embedding.

Proof. $f \mapsto[f]$ is an order embedding, because in a Baire space the comeager sets are dense. It is a monoid homomorphism because, as was observed in 4.4 , ( $\left.\operatorname{Baire}\left(X, \mathbb{N}^{I}\right) /=_{\mathrm{aa}},+\right)$ is a quotient monoid. And it is a lattice homomorphism by equation (4.37).

Lemma 4.69. Let $X$ be a zero dimensional space. Then $C\left(X, \mathbb{N}^{I}\right)$ is dense in the quasi order $\left(\operatorname{Baire}\left(X, \mathbb{N}^{I}\right), \leq_{\mathrm{aa}}\right)$. 
Proof. Take $f \in \operatorname{Baire}\left(X, \mathbb{N}^{I}\right)$ with $[f] \neq\left[\mathbf{0}_{I-}\right]$. Then for some $i \in I, f^{i}=_{\text {naa }} 0$ (i.e. not $f^{i}={ }_{\text {aa }} 0$ ), and thus there exists $n=1,2, \ldots$ such that $B=\{z \in X$ : $f(z)(i)=n\} \notin \mathcal{M}(X)$. Since $X$ is zero dimensional, there exists a nonempty clopen set $C$ with $C \backslash B \in \mathcal{M}(X)$. Now $\left\langle C, \chi_{i}\right\rangle \in C\left(X, \mathbb{N}^{I}\right)^{+}$(cf. notation 4.54) since $C$ is clopen, and clearly $\left\langle C, \chi_{i}\right\rangle \leq_{\text {aa }} f$.

Corollary 4.70. Let $X$ be a zero dimensional Baire space. Then $C\left(X, \mathbb{N}^{I}\right)$ densely embeds into (Baire $\left(X, \mathbb{N}^{I}\right), \leq_{\mathrm{aa}}$ ).

Proof. Proposition 4.68 and lemma 4.69.

Example 4.71. The irrationals $\mathbb{N}^{\mathbb{N}}$ form a zero dimensional Baire space, and indeed this is often called the Baire space. More generally, so is $\mathbb{N}^{H}$ for any $H$, and thus $C\left(\mathbb{N}^{H}, \mathbb{N}^{I}\right)$ densely embeds into Baire $\left(\mathbb{N}^{H}, \mathbb{N}^{I}\right) /=$ aa.

The embedding is generally not onto.

Example 4.72. Since the irrationals of the real line $\mathbb{R} \backslash \mathbb{Q}$ are homeomorphic to our set of irrationals $\mathbb{N}^{\mathbb{N}}, C(\mathbb{R} \backslash \mathbb{Q}, \mathbb{R} \backslash \mathbb{Q})$ densely embeds into Baire $(\mathbb{R} \backslash \mathbb{Q}, \mathbb{R} \backslash \mathbb{Q}) /=$ aa (we can make sense of the latter using the fact that $=_{a a}$ is the same as $\{=\}_{\text {aa }}$ on $\mathbb{N}^{\mathbb{N}}$ ). Now fix an irrational number $\sigma \in \mathbb{R}$. Then a jump function $f: \mathbb{R} \backslash \mathbb{Q} \rightarrow \mathbb{R} \backslash \mathbb{Q}$ where

$$
f(x)= \begin{cases}0, & \text { if } x \leq \sigma, \\ 1, & \text { if } x>\sigma,\end{cases}
$$

is a Baire measurable function, but no function $g: \mathbb{R} \backslash \mathbb{Q} \rightarrow \mathbb{R} \backslash \mathbb{Q}$ with $g={ }_{\text {aa }} f$ is continuous at $\sigma$. Therefore there is a function $f \in \operatorname{Baire}\left(\mathbb{N}^{\mathbb{N}}, \mathbb{N}^{\mathbb{N}}\right)$ with no member of $C\left(\mathbb{N}^{\mathbb{N}}, \mathbb{N}^{\mathbb{N}}\right)$ in its equivalence class modulo $=$ aa.

Proposition 4.73. Let $X$ be a Baire space. Then $C\left(X, \mathbb{N}^{I}\right)$ identifies (via $f \mapsto[f]$ ) with a submonoid of $\left(\right.$ Baire $\left.\left(X, \mathbb{N}^{I}\right) /={ }_{\mathrm{aa}},+\right)$ that is closed under subtraction. It also identifies with a sublattice of $\left(\operatorname{Baire}\left(X, \mathbb{N}^{I}\right) /={ }_{\mathrm{aa}}, \leq \mathrm{aa}\right)$.

Proof. Propositions 4.67 and 4.68 .

When $X$ is a zero dimensional Baire space, $C\left(X, \mathbb{N}^{I}\right)$ identifies with a subset of $\operatorname{Baire}\left(X, \mathbb{N}^{I}\right) /=_{\text {aa }}$ with several nice properties.

Lemma 4.74. Let $X$ be a zero dimensional Baire space. Then $C\left(X, \mathbb{N}^{I}\right)$ identifies (via $f \mapsto[f]$ ) with a subset of the lattice monoid (Baire $\left(X, \mathbb{N}^{I}\right) /={ }_{\text {aa }},+$ ) satisfying the following properties: It is regular, it forms a basis and it is strongly interval predense.

Proof. First we show that $C\left(X, \mathbb{N}^{I}\right)$ is (i.e. identifies with) a basis. Since the basic elements form a basis by proposition 4.47, it suffices to prove that each basic element $\left\langle a, n \cdot \chi_{i}\right\rangle$ is the supremum of an antichain of $C\left(X, \mathbb{N}^{I}\right)$. And this is so because it is the supremum of an antichain of elements of $C\left(X, \mathbb{N}^{I}\right)$ of the form $\left\langle C, n \cdot \chi_{i}\right\rangle$ with $C \subseteq X$ clopen, by example 3.67 .

We already know that $\left(\operatorname{Baire}\left(X, \mathbb{N}^{I}\right) /={ }_{a a},+\right)$ is a cancellative commutative monoid. Therefore, $C\left(X, \mathbb{N}^{I}\right)$ is a regular subset by lemma 3.16, because it is a dense submonoid closed under subtraction by corollary 4.70 and proposition 4.73 , 
In theorem 4.40 (see also remark 4.41), it was established that Baire $\left(X, \mathbb{N}^{I}\right) /=$ aa is moreover a complete semilattice monoid satisfying the (JID). Therefore, since we have shown that $C\left(X, \mathbb{N}^{I}\right)$ is a submonoid forming a basis, it is strongly interval predense by lemma 3.71 .

Lemma 4.75. Let $X$ be a zero dimensional Baire space, and let $M$ be a complete semilattice that is flat-complete and (JID). Then every order embedding of $C\left(X, \mathbb{N}^{I}\right)$ into $M$ with preregular range uniquely extends to a continuous lattice embedding of $\operatorname{Baire}\left(X, \mathbb{N}^{I}\right) /=_{\text {aa }}$ into $M$.

Proof. We know that Baire $\left(X, \mathbb{N}^{I}\right) /=_{\text {aa }}$ is a (JID) lattice. By lemma 4.74, $C\left(X, \mathbb{N}^{I}\right)$ forms a strongly interval predense basis. Hence every order embedding of $C\left(X, \mathbb{N}^{I}\right)$ into $M$ with preregular range has an extension, unique on $C\left(X, \mathbb{N}^{I}\right)^{+}$, to a continuous lattice embedding by corollary 3.94 (or alternatively, by corollary 3.95). And the extension is in fact unique because $C\left(X, \mathbb{N}^{I}\right)$, being a submonoid, contains the zero of Baire $\left(X, \mathbb{N}^{I}\right) /={ }_{\text {aa }}$ (cf. remark (3.86).

Theorem 4.76. Let $X$ and $Y$ be a zero dimensional Baire spaces. Then every order embedding of $C\left(X, \mathbb{N}^{I}\right)$ into $C\left(Y, \mathbb{N}^{J}\right)$ with convex range has a unique extension to a continuous lattice embedding of $\operatorname{Baire}\left(X, \mathbb{N}^{I}\right) /={ }_{\mathrm{aa}}$ into $\operatorname{Baire}\left(Y, \mathbb{N}^{J}\right) /={ }_{\mathrm{aa}}$ with convex range.

Proof. We know that $\operatorname{Baire}\left(X, \mathbb{N}^{I}\right) /={ }_{\text {aa }}$ is a cancellative commutative complete semilattice monoid satisfying the (JID), and so is $\operatorname{Baire}\left(Y, \mathbb{N}^{J}\right) /={ }_{\text {aa }}$, and they are moreover flat-complete by theorem 4.40. Since $C\left(X, \mathbb{N}^{I}\right)$ is a submonoid forming a basis and $C\left(Y, \mathbb{N}^{J}\right)$ is a dense submonoid closed under subtraction, the conclusion is by corollary 3.98 ,

Theorem 4.77. Let $X$ be a regular zero dimensional Baire space, and let $Y$ be a zero dimensional space with a Baire compactification. The order embeddings from $C\left(X, \mathbb{N}^{I}\right)$ into $C\left(Y, \mathbb{N}^{J}\right)$ with convex range consist of maps of the form

$$
\sigma=\pi_{g, H} \frown \mathbf{0}_{J \backslash K-}+h
$$

for some $K \in C(Y, \mathcal{P}(J)), g \in C\left(I^{K-}\right), H \in C\left(X^{K-}\right)$ such that

$$
\begin{gathered}
\left(H^{j}\right)^{-1}[M] \in \mathcal{M}(Y) \quad \text { for all } M \in \mathcal{M}(X) \text { and all } j \in J, \\
\left(H^{j}\right)^{-1}[M] \cap\left\|g^{j}=i\right\| \in \mathcal{M}(Y) \quad \text { implies } \quad M \cap H^{j}\left[\left\|g^{j}=i\right\|\right] \in \mathcal{M}(X)
\end{gathered}
$$

for every $i \in I$ and $M \subseteq X$,

$$
\begin{gathered}
H^{j}\left[\left\|g^{j}=i\right\|\right] \cap H^{j^{*}}\left[\left\|g^{j^{*}}=i\right\|\right]=\emptyset \quad \text { for all } j \neq j^{*} \text { in } J, \text { and all } i \in I, \\
\bigvee_{j \in J}\left[H^{j}\left[\left\|g^{j}=i\right\|\right]\right]_{\operatorname{Cat}(X)}=1_{\operatorname{Cat}(X)} \quad \text { for all } i \in I,
\end{gathered}
$$

and some $h \in C\left(Y, \mathbb{N}^{J}\right)$. Moreover, the range is given by

$$
\operatorname{ran}(\sigma)=C\left(Y, \mathbb{N}^{K}\right) \frown \mathbf{0}_{J \backslash K-}+h .
$$


Proof. Let $\sigma: C\left(X, \mathbb{N}^{I}\right) \rightarrow C\left(Y, \mathbb{N}^{J}\right)$ be an order embedding with convex range. Then, noting that $Y$ is a Baire space (proposition 4.21), theorem 4.76 gives a unique embedding $\bar{\sigma}$ of $\operatorname{Baire}\left(X, \mathbb{N}^{I}\right) /={ }_{\text {aa }}$ into $\operatorname{Baire}\left(Y, \mathbb{N}^{J}\right) /={ }_{\text {aa }}$ with convex range, such that

$$
\bar{\sigma}([f])=[\sigma(f)] \text { for all } f \in C\left(X, \mathbb{N}^{I}\right) .
$$

Now we apply theorem 4.62 to obtain $K \in \operatorname{Baire}(Y, \mathcal{P}(J)), g \in C\left(I^{K-}\right), H \in$ $\operatorname{Baire}\left(X^{K-}\right)$ and $h \in \operatorname{Baire}\left(Y, \mathbb{N}^{J}\right)$ satisfying equations (4.102)-(4.105), and $\bar{\sigma}=$ $\left[\pi_{g, H} \frown \mathbf{0}_{J \backslash K-}+h\right]$. Now also equation (4.101) follows from (4.107). It remains to show that $K$ and $h$ are in fact continuous, and that $H \in C\left(X^{K-}\right)$.

We see that $h$ is continuous by plugging $\mathbf{0}_{I-}$ into (4.101). Thus, by equation (4.101) and closure under subtraction (proposition 4.67),

$$
\pi_{g, H}(f) \frown \mathbf{0}_{J \backslash K-} \text { is continuous for all } f \in C\left(X, \mathbb{N}^{I}\right) .
$$

We can use equation (4.81) to see that $\pi_{g, H}\left(\mathbf{1}_{I-}\right)=\mathbf{1}_{K-} \frown \mathbf{0}_{J \backslash K-}$. If $K$ is not continuous, then clearly neither is $\mathbf{1}_{K-} \frown \mathbf{0}_{J \backslash K-}$, contradicting (4.108) with $f=\mathbf{1}_{I-}$.

Suppose now that $H \notin C\left(X^{K-}\right)$. Then for some $j \in J, H^{j}$ is noncontinuous at some $z_{0} \in\|j \in K\|$, say $U \ni H^{j}\left(z_{0}\right)$ is an open subset of $X$ and $H^{j}[V] \nsubseteq U$ for every open $V \ni z_{0}$. Since $g^{j}$ is continuous, there exists an open $V \ni z_{0}$ with $g^{j}(z)=i$ for all $z \in V$. Since $C\left(X, \mathbb{N}^{I}\right)$ is dense (corollary 4.70), we can find a continuous $f: X \rightarrow \mathbb{N}^{I}$ such that $f\left(H^{j}\left(z_{0}\right)\right)(i)=1$ but $f\left(H^{j}(z)\right)(i)=0$ for all $z \notin U$. Now $\pi_{g, H}(f) \frown \mathbf{0}_{J \backslash K-}$ is not continuous at $z_{0}$ contrary to (4.108), because $\pi_{g, H}(f)\left(z_{0}\right)(j)=1$ but every open $V \supseteq V^{\prime} \ni z_{0}$ has a $z \in V^{\prime}$ with $H^{j}(z) \notin U$ and thus $\pi_{g, H}(f)(z)(j)=0$.

For the converse, it is clear that $\pi_{g, H}(f)$ is continuous for all $f \in C\left(X, \mathbb{N}^{I}\right)$, whenever $K$ and is continuous, $g \in C\left(I^{K-}\right)$ and $H \in C\left(X^{K-}\right)$. And thus it follows from theorem 4.62 that equation (4.101) determines an order embedding. As for having convex range, this is automatic once equation (4.106) is established. However, one can use the properties (4.102)-(4.105) to prove that $\pi_{g, H}(f)-\mathbf{0}_{J \backslash K \text { - }}$ is noncontinuous whenever $f$ is noncontinuous. Thus $\operatorname{ran}(\sigma)$ is equal to the intersection of $C\left(X, \mathbb{N}^{I}\right)$ with the set of representatives of $\operatorname{ran}(\bar{\sigma})$. Now equation (4.106) is an immediate consequence of equation (4.87).

4.6. Further directions. We suggest some classes of partial orders (out of infinitely many possibilities) for which we feel it would be interesting to know what the partial order embeddings with convex range are.

4.6.1. Baire $(X, \mathcal{P}(S)) /={ }_{\text {aa }}$. In $\$ 4.1$ we examined embeddings with convex range between arbitrary power set algebras. We expect that this can be generalized to the quotient of Baire functions into power set algebras modulo almost always equality, in a completely analogous manner to $\$ 4.4$.

Fixing some set $S$, the natural analogue of $={ }_{\text {aa }}$ for $\mathcal{P}(S)$ is given by the family $\left\{R_{s}: s \in S\right\}$ where $x R_{s} y$ iff $(s \in x$ iff $s \in y)$. And $\subseteq_{\text {aa }}$ is defined analogously. We should then be able to go through the same analysis for embeddings between posets of the form (Baire $\left.(X, \mathcal{P}(S)) /={ }_{\text {aa }}, \subseteq_{\text {aa }}\right)$, to obtain analogues of theorems 4.50 
and 4.62. Also note that one obtains an analogous connection with set theoretic forcing, e.g. for $f, g \in \operatorname{Baire}(X, \mathcal{P}(S))$,

$$
\begin{array}{lll}
\operatorname{Cat}(X) \Vdash \dot{f}=\dot{g} & \text { iff } & f={ }_{\text {aa }} g, \\
\operatorname{Cat}(X) \Vdash \dot{f} \subseteq \dot{g} & \text { iff } & f \subseteq \text { aa } g .
\end{array}
$$

4.6.2. $L^{0}\left(\mu, \mathbb{N}^{I}\right) /={ }_{\text {ae }}$. Let $(X, \mu)$ be a measure space, and $S$ a topological space. For simplicity let us insist that the measure is totally finite (i.e. $\mu(X)<\infty$ ) or at least $\sigma$-finite. Then we denote the family of all $\mu$-measurable functions from $X$ into $Y$ by $L^{0}(\mu, S)$, i.e. functions $f: X \rightarrow S$ such that $f^{-1}[U]$ is in the domain of the measure $\mu$ for every open $U \subseteq S$. Then analogously to the families of Baire measurable functions, for a set of relations $\mathcal{R}$ on $S$, we define a relation $\mathcal{R}_{\text {ae }}$ on $L^{0}(\mu, S)$ by

$$
f \mathcal{R}_{\text {ae }} g \text { if } \bigwedge_{R \in \mathcal{R}}\ulcorner f(z) R g(z) \text { for almost every } z \in X\urcorner,
$$

where "for almost every" is interpreted as the complement of a measure zero subset of $X$. Using the same sets of relations as in example 4.35, we obtain $\leq_{\mathrm{ae}}$ and $=a \mathrm{ae}$. And then we can form the quotient poset $\left(L^{0}\left(\mu, \mathbb{N}^{I}\right) /=_{\mathrm{ae}}, \leq_{\mathrm{ae}}\right)$. We have now obtained the measure theoretic analogue of the quotient lattice of $\$ 4.4$. We again have an analogue with set theoretic forcing, but now our forcing notion is the complete Boolean algebra $\operatorname{Ran}(\mu)=\operatorname{dom}(\mu) / \mathcal{N}_{\mu}$ where $\mathcal{N}_{\mu}$ denotes the ideal of $\mu$ measure zero subsets of $X$ (i.e. we are forcing with a measure algebra; more precisely, $(\operatorname{Ran}(\mu), \nu)$, with $\nu([a])=\mu(a)$, is the measure algebra of the measure space $(X, \mu))$. This is known as random forcing and was invented by Solovay [Sol70] to prove the consistency of all subsets of the real line being Lebesgue measurable with the usual axioms of mathematics minus the Axiom of Choice. By way of analogy, we have e.g. for all $f, g \in L^{0}\left(\mu, \mathbb{N}^{I}\right)$,

$$
\begin{array}{lll}
\operatorname{Ran}(\mu) \Vdash \dot{f}=\dot{g} & \text { iff } & f={ }_{\text {ae }} g, \\
\operatorname{Ran}(\mu) \Vdash \dot{f} \leq \dot{g} & \text { iff } & f \leq \text { ae } g
\end{array}
$$

(see e.g. [Hir00b]).

Moreover, theorem 4.50 also describes the order embeddings between $L^{0}\left(\mu, \mathbb{N}^{I}\right) /$ $={ }_{\mathrm{ae}}$ and $L^{0}\left(\nu, \mathbb{N}^{J}\right) /={ }_{\text {ae }}$ with convex range, by replacing $\operatorname{Cat}(X)$ and $\operatorname{Cat}(Y)$ with $\operatorname{Ran}(\mu)$ and $\operatorname{Ran}(\nu)$, respectively. In fact, the proof of theorem 4.50 does not use properties specific to $\operatorname{Cat}(X)$ other than completeness; and this theorem can be generalized to arbitrary quotients of families of functions measurable with respect to some $\sigma$-ideal on a fixed set $X$, provided the ideal satisfies the countable chain condition, and both the meager ideal and the ideal of $\mu$-measure zero sets satisfy this property.

The question here is whether we can obtain a measure theoretic analogue of theorem 4.62,

Question 1. Supposing that $(X, \mu)$ and $(Y, \nu)$ are 'reasonable' measure spaces, can we describe the order embeddings of $L^{0}\left(\mu, \mathbb{N}^{I}\right) /={ }_{\text {ae }}$ into $L^{0}\left(\nu, \mathbb{N}^{J}\right) /={ }_{\text {ae }}$ with convex 
range, in an analogous way to theorem 4.62, using measurable functions $K: Y \rightarrow$ $\mathcal{P}(J), g: Y \rightarrow I^{K-}, H: Y \rightarrow X^{K-}$ and $h: Y \rightarrow \mathbb{N}^{J}$ ?

4.6.3. $\left(\mathcal{P}(\mathbb{N}) /\right.$ Fin, $\left.\subseteq^{*}\right)$. Consider now the power set $\mathcal{P}(\mathbb{N})$ of $\mathbb{N}$ quasi ordered by "almost inclusion", i.e. $a \subseteq^{*} b$ if $a \backslash b$ is finite. The quotient $\left(\mathcal{P}(\mathbb{N}) /\right.$ Fin, $\left.\subseteq^{*}\right)$ over the equivalence relation $=^{*}$ given by $a=^{*} b$ if $a \subseteq^{*} b$ and $b \subseteq^{*} a$ (i.e. $a \triangle b$ is finite) is a Boolean algebra of great importance in a number of areas of mathematics. In fact, there is a general program of research aimed at investigating the class of partial orders that embed into $\mathcal{P}(\mathbb{N}) /$ Fin, to which the present paper is clearly relevant. To give one example, this class of orders played a major role in the solution, by SolovayWoodin (see [DW87]), of a famous problem of Kaplansky [Kap49] on automatic continuity in Banach algebras.

The structure of the order automorphism group (equivalently, Boolean algebra automorphisms) is independent of the usual axioms of mathematics, as was established by Shelah in his celebrated result [She82]:

Theorem 4.78 (Shelah). It is consistent that every order automorphism of $(\mathcal{P}(\mathbb{N}) /$ Fin, $\left.\subseteq^{*}\right)$ is trivial.

A trivial automorphism of $\left(\mathcal{P}(\mathbb{N}) / \mathrm{Fin}, \subseteq^{*}\right)$ is one of the form

$$
\sigma([a])=[h[a]]
$$

for some bijection $h: A \rightarrow B$ where $A, B \subseteq \mathbb{N}$ are both cofinite (see also below). This should be compared to corollary 4.4. Note that it was already known that there exist nontrivial automorphisms under $\mathrm{CH}$. On the other hand, it is a consequence of Shelah's theorem that no nontrivial automorphism can have a simple (e.g. Borel) definition.

We do not know if for example Shelah's result has been extended to say Boolean algebra homomorphisms (or even monomorphisms or epimorphisms). Putting this in the framework of this paper, we obtain an even more general question.

Question 2. What are the order embeddings of $\mathcal{P}(\mathbb{N}) /$ Fin into itself with convex range? Is it consistent that they are all trivial?

In question 2 the precise meaning of trivial is a mapping $[a] \mapsto[h[a] \cup[b]]$ where $h: A \rightarrow \mathbb{N}$ is an injection with $A$ cofinite and $b \cap \operatorname{ran}(h)$ finite.

4.6.4. $\left(\mathbb{N}^{\mathbb{N}} /\right.$ Fin,$\left.\leq^{*}\right)$. The eventual dominance quasi ordering $\leq^{*}$ of the irrationals $\mathbb{N}^{\mathbb{N}}$ is given by $x \leq^{*} y$ if $x(n) \leq y(n)$ for all but finitely many $n \in \mathbb{N}$. The quotient $\left(\mathbb{N}^{\mathbb{N}} /\right.$ Fin, $\left.\leq^{*}\right)$ over the equivalence relation $=^{*}$ given by $x={ }^{*} y$ if $x \leq^{*} y$ and $y \leq^{*} x$ (i.e. $x(n)=y(n)$ for all but finitely many $n$ ) is a lattice. Analogously to $\mathcal{P}(\mathbb{N}) /$ Fin we can formulate the notion of a "trivial" order embedding.

Definition 4.79. An order endomorphism from $\mathbb{N}^{\mathbb{N}} /$ Fin into itself is called trivial if it is of the form

$$
\sigma([x])=\left[\pi_{g}(x) \frown \mathbf{0}_{\mathbb{N} \backslash \operatorname{dom}(g)}+y\right]
$$

for some finite-one $g: A \rightarrow \mathbb{N}$, and some $y \in \mathbb{N}^{\mathbb{N}}$. 
Proposition 4.80. Every such trivial mapping is indeed an order preserving map between $\left(\mathbb{N}^{\mathbb{N}} /\right.$ Fin, $\left.\leq^{*}\right)$ and itself.

Proposition 4.81. A trivial endomorphism as in equation (4.115) is an embedding iff $\operatorname{ran}(g)$ is cofinite.

Proposition 4.82. A trivial endomorphism has convex range iff it can be represented by equation (4.115) with an injection $g$.

Proposition 4.83. A trivial endomorphism is an epimorphism iff it can be represented by equation (4.115) with $A$ cofinite and $g$ an injection.

Therefore, every trivial embedding of $\mathbb{N}^{\mathbb{N}} /$ Fin into itself with convex range is of the form $[x] \mapsto\left[\pi_{g}(x)^{\frown} \mathbf{0}_{\mathbb{N} \backslash \operatorname{dom}(g)}+y\right]$ where $g: A \rightarrow \mathbb{N}$ is an injection with cofinite range and $y \in \mathbb{N}^{\mathbb{N}}$. And it follows that every trivial automorphism is of the form $[x] \mapsto\left[\pi_{g}(x)^{\frown} \mathbf{0}_{\mathbb{N} \backslash \operatorname{dom}(g)}\right]$ where $g: A \rightarrow B$ is a bijection and $A$ and $B$ are both cofinite subsets of $\mathbb{N}$.

We could not find any result in the literature for $\mathbb{N}^{\mathbb{N}} /$ Fin corresponding to Shelah's theorem, nor did we attempt to construct a nontrivial endomorphism.

Question 3. Is the existence of a nontrivial automorphism of $\left(\mathbb{N}^{\mathbb{N}} /\right.$ Fin, $\left.\leq^{*}\right)$ consistent? If so, is it consistent that every automorphism of $\mathbb{N}^{\mathbb{N}} /$ Fin is trivial?

Putting question 3 into the framework of embeddings with convex range, we obtain the following generalization.

Question 4. What are the order embeddings of $\mathbb{N}^{\mathbb{N}} /$ Fin into itself with convex range? Is it consistent that they are all trivial?

[AJ94] Samson Abramsky and Achim Jung, Domain theory, Handbook of logic in computer science, Vol. 3, Oxford Univ. Press, New York, 1994, pp. 1-168. (97b:68121) 个3.5, 3.2 $3.3,3.3$

[Coh63] Paul J. Cohen, The independence of the continuum hypothesis, Proc. Nat. Acad. Sci. U.S.A. 50 (1963), 1143-1148. $(28 \# 1118) \uparrow 4.4$

[Coh64] , The independence of the continuum hypothesis. II, Proc. Nat. Acad. Sci. U.S.A. 51 (1964), 105-110. (28 \#2962) $\uparrow 4.4$

[DP02] B. A. Davey and H. A. Priestley, Introduction to lattices and order, second ed., Cambridge University Press, New York, 2002. (2003e:06001) $\uparrow$ 1.1, 2.1.1, 3.21, 3.65

[DR81] Dwight Duffus and Ivan Rival, A structure theory for ordered sets, Discrete Math. 35 (1981), 53-118. (82f:06001) $\uparrow 1.1$

[DW87] H. G. Dales and W. H. Woodin, An introduction to independence for analysts, London Mathematical Society Lecture Note Series, vol. 115, Cambridge University Press, Cambridge, 1987. (90d:03101) $\uparrow 4.6 .3$

[Eng97] Konrad Engel, Sperner theory, Encyclopedia of Mathematics and its Applications, vol. 65, Cambridge University Press, Cambridge, 1997. (98m:05187) $\uparrow 3.65$

[Hir00a] James Hirschorn, Towers of Borel functions, Proc. Amer. Math. Soc. 128 (2000), no. 2, 599-604. (2000c:03040) 个4.41

[Hir00b] —, Towers of measurable functions, Fund. Math. 164 (2000), no. 2, 165-192. (2002i:03056) $\uparrow 4.6 .2$

[Hir06a] , Characterizing the ordering of the irrationals by eventual dominance, in progress, 2006. $\uparrow 1,4.4$ 
[Hir06b] _ Pinning quasi orders with their endomorphisms, arXiv:math.RA/0612495, 2006. http://homepage.univie.ac.at/James.Hirschorn/research/pinning/pinning.html $\uparrow$ 11

[Kan69] P1. Kannappan, Characterizing topology on an Abelian semigroup by a functional equation, Portugal. Math. 28 (1969), 97-101. (44 \#2862) 个2

[Kap49] Irving Kaplansky, Normed algebras, Duke Math. J. 16 (1949), 399-418. (11,115d) 个4.6.3

[Kec95] Alexander S. Kechris, Classical descriptive set theory, Graduate Texts in Mathematics, vol. 156, Springer-Verlag, New York, 1995. (96e:03057) 个4.3

[Kop89] Sabine Koppelberg, Handbook of Boolean algebras. Vol. 1, North-Holland Publishing Co., Amsterdam, 1989, Edited by J. Donald Monk and Robert Bonnet. (90k:06002) 个 2.1.1 3.13 .4

[Kun80] Kenneth Kunen, Set theory, Studies in Logic and the Foundations of Mathematics, vol. 102, North-Holland Publishing Co., Amsterdam, 1980, An introduction to independence proofs. (82f:03001) $\uparrow 4.4$

[Sco67] Dana Scott, A proof of the independence of the continuum hypothesis, Math. Systems Theory 1 (1967), 89-111. (36 \#1321) 个4.4

[She82] Saharon Shelah, Proper forcing, Lecture Notes in Mathematics, vol. 940, Springer-Verlag, Berlin, 1982. (84h:03002) 个4.6.3

[She98] _ Proper and improper forcing, second ed., Perspectives in Mathematical Logic, Springer-Verlag, Berlin, 1998. (98m:03002) 个4.1

[Smy92] M. B. Smyth, Topology, Handbook of logic in computer science, Vol. 1, Oxford Univ. Press, New York, 1992, pp. 641-761. (97i:68131) 个3.3

[Sol70] Robert M. Solovay, A model of set-theory in which every set of reals is Lebesgue measurable, Ann. of Math. (2) 92 (1970), 1-56. (42 \#64) 个4.6.2

[Weh92] Friedrich Wehrung, Injective positively ordered monoids. I, II, J. Pure Appl. Algebra 83 (1992), no. 1, 43-82, 83-100. (93k:06023) 个2

Graduate School of Science and Technology, Kobe University, Japan

E-mail address: j_hirschorn@yahoo.com

URL: http://www.logic.univie.ac.at/ hirschor/ 